TRANSACTIONS OF THE

AMERICAN MATHEMATICAL SOCIETY

Volume 355, Number 5, Pages 1729-1772

S 0002-9947(03)03202-1

Article electronically published on January 8, 2003

\title{
EXTENDER-BASED RADIN FORCING
}

\author{
CARMI MERIMOVICH
}

\begin{abstract}
We define extender sequences, generalizing measure sequences of Radin forcing.

Using the extender sequences, we show how to combine the Gitik-Magidor forcing for adding many Prikry sequences with Radin forcing.

We show that this forcing satisfies a Prikry-like condition, destroys no cardinals, and has a kind of properness.

Depending on the large cardinals we start with, this forcing can blow the power of a cardinal together with changing its cofinality to a prescribed value. It can even blow the power of a cardinal while keeping it regular or measurable.
\end{abstract}

\section{INTRODUCTION}

We give some background on previous work relating directly to the present work. The first forcing which changed the cofinality of a cardinal without changing the cardinal structure was Prikry forcing [6]. In this forcing a measurable cardinal, $\kappa$, was 'invested' in order to get $\operatorname{cf}(\kappa)=\omega$ without collapsing any cardinal. Developing that idea, Magidor [2] used a coherent sequence of measures of length $\lambda<\kappa$ in order to get $\operatorname{cf}(\kappa)=\lambda$ without collapsing any cardinals. In [7] Radin, introducing the notion of measure sequence, showed that it is useful to continue the coherent sequence to $\lambda>\kappa$. For example, $\kappa$ remains regular when $\lambda=\kappa^{+}$. In general, the longer the measure sequence, the more resemblance there is between $\kappa$ in the generic extension and the ground model.

As is well known, and unlike regular cardinals, blowing the power of a singular cardinal is not an easy task. A natural approach to try was to blow the power of a cardinal while it was regular and after that make it singular by one of the above methods. A crucial idea of Gitik and Magidor [3] was to combine the power set blowing and the cofinality change in one forcing. They introduced a forcing notion which added many Prikry sequences at once and still collapsed no cardinals. The 'investment' they needed for this was an extender of length which is the size of the power they wanted. Building on the idea of Gitik and Magidor, Segal [8] implemented the idea of adding many sequences to Magidor forcing. So by investing

Received by the editors October 19, 1998.

2000 Mathematics Subject Classification. Primary 03E35, 03 E55.

Key words and phrases. Forcing, Radin forcing, extender, extender-based forcing, generalized continuum hypothesis, singular cardinal hypothesis.

This work is a part of research which, hopefully, will become the author's Ph.D. thesis. It was done at Tel-Aviv University under the supervision of M. Gitik. The author thanks M. Gitik for his help with this work, with other works and just in general.

We thank Asaf Sharon for reading and pointing out some problems in a preliminary version of this work. 
a coherent sequence of extenders of length $\lambda<\kappa$ she was able to get a singular cardinal of cofinality $\lambda$ together with power as large as the length of the extenders in question. Our work also builds on the idea of Gitik and Magidor. However, we implement the idea of adding many sequences to Radin forcing. So we introduce the notion of extender sequence and show that it makes sense to deal with quite long extender sequences. As in Radin forcing, for long enough sequences we are left with $\kappa$ which is regular and even measurable. The power size will be the length of the extenders we start with.

The structure of this work is as follows. In section 2 we define extender sequences. In section 3 we define $P_{\bar{E}}$, the forcing notion which is the purpose of this work. In section 4 we define Radin forcing as a means to prove lemmas we need later. The definition is not the usual one. The definition we give here is more in the spirit of the forcing we defined in section 3 . In section 5 we show the chain-conditions satisfied by $P_{\bar{E}}$ and how 'locally' it resembles Radin forcing. We also show here that there are many new subsets in the generic extension. In section [6 we investigate the structure of dense open subsets of $P_{\bar{E}}$. We show that they satisfy a strong homogeneity property. In section 7 we prove Prikry's condition for $P_{\bar{E}}$. The proof is a simple corollary of the strong homogeneity of dense open subsets. In section 8 we show that $P_{\bar{E}}$ satisfies a kind of properness. In section 9 we combine the machinery developed so far in order to show that no cardinals are collapsed. In section 10 we show how the length of the extender sequences affects the properties of $\kappa$. Section 11 summarizes what the forcing $P_{\bar{E}}$ does. In section 12 we have a result concerning $P_{\bar{E}}$ when $\mathrm{l}(\bar{E})=1$. We show that there is, in $V$, a generic filter over an elementary submodel in an $\omega$-iterate of $V$. We were not able to prove something equivalent (or weaker) for the general case. Section[13 contains a list of missing or unknown points to check. The last point in this list is in preparation.

Our notation is standard. We assume fluency with forcing and extenders. Some basic properties of Radin forcing are taken for granted.

\section{Extender Sequences}

2.1. Constructing from elementary embedding. Suppose we have an elementary embedding $j: V \rightarrow M \supset V_{\lambda}$, $\operatorname{crit}(j)=\kappa$. The value of $\lambda$ will be determined later, according to the different applications we have.

Construct from $j$ a nice extender such as in [3]:

$$
E(0)=\left\langle\left\langle E_{\alpha}(0) \mid \alpha \in \mathcal{A}\right\rangle,\left\langle\pi_{\beta, \alpha} \mid \beta \geq_{\mathcal{A}} \alpha \alpha, \beta \in \mathcal{A}\right\rangle\right\rangle .
$$

We recall the properties of this extender:

(1) $\mathcal{A} \subseteq\left|V_{\lambda}\right| \backslash \kappa$

(2) $|\mathcal{A}|=\left|V_{\lambda}\right|$

(3) $\left\langle\mathcal{A}, \leq_{\mathcal{A}}\right\rangle$ is a $\kappa^{+}$-directed partial order,

(4) $\forall \alpha, \beta \in \mathcal{A} \beta \geq_{\mathcal{A}} \alpha \Longrightarrow \pi_{\beta, \alpha}: V_{\kappa} \rightarrow V_{\kappa}$,

(5) $\kappa \in \mathcal{A}$,

(6) $\forall \alpha \in \mathcal{A} \kappa \leq_{\mathcal{A}} \alpha$. We write $\pi_{\alpha, 0}$ instead of $\pi_{\alpha, \kappa}$,

(7) $\forall \alpha, \beta \in \mathcal{A} \forall \nu<\kappa \nu^{0}=\pi_{\alpha, 0}(\nu)=\pi_{\beta, 0}(\nu)$,

(8) $\forall \alpha, \beta \in \mathcal{A} \beta \geq_{\mathcal{A}} \alpha \Longrightarrow \forall \nu<\kappa \pi_{\beta, 0}(\nu)=\pi_{\alpha, 0}\left(\pi_{\beta, \alpha}(\nu)\right)$,

(9) $\forall \alpha, \beta, \gamma \in \mathcal{A} \gamma \geq_{\mathcal{A}} \beta \geq_{\mathcal{A}} \alpha \Longrightarrow$

$$
\exists A \in E_{\gamma}(0) \forall \nu \in A \pi_{\gamma, \alpha}(\nu)=\pi_{\beta, \alpha}\left(\pi_{\gamma, \beta}(\nu)\right) .
$$


If, for example, we need $|E(0)|=\kappa^{+3}$, then, under GCH, we require $\lambda=\kappa+3$. A typical large set in this extender concentrates on singletons.

If $j$ is not sufficiently closed, then $E(0) \notin M$ and the construction stops. We set

$$
\forall \alpha \in \mathcal{A} \bar{E}_{\alpha}=\langle\alpha, E(0)\rangle \text {. }
$$

We say that $\bar{E}_{\alpha}$ is an extender sequence of length $1\left(l\left(\bar{E}_{\alpha}\right)=1\right)$.

If, on the other hand, $E(0) \in M$, we can construct for each $\alpha \in \operatorname{dom} E(0)$ the following ultrafilter:

$$
A \in E_{\langle\alpha, E(0)\rangle}(1) \Longleftrightarrow\langle\alpha, E(0)\rangle \in j(A) .
$$

Such an $A$ concentrates on elements of the form $\langle\xi, e(0)\rangle$ where $e(0)$ is an extender on $\xi^{0}$ and $\xi \in \operatorname{dom} e(0)$. Note that $e(0)$ concentrates on singletons below $\xi^{0}$. If, for example, $|E(0)|=\kappa^{+3}$, then on a large set we have $|e(0)|=\left(\xi^{0}\right)^{+3}$.

We define $\pi_{\langle\beta, E(0)\rangle,\langle\alpha, E(0)\rangle}$ as

$$
\pi_{\langle\beta, E(0)\rangle,\langle\alpha, E(0)\rangle}(\langle\xi, e(0)\rangle)=\left\langle\pi_{\beta, \alpha}(\xi), e(0)\right\rangle .
$$

From this definition we get

$$
j\left(\pi_{\langle\beta, E(0)\rangle,\langle\alpha, E(0)\rangle}\right)(\langle\beta, E(0)\rangle)=\langle\alpha, E(0)\rangle .
$$

Hence we have here an extender

$$
E(1)=\left\langle\left\langle E_{\langle\alpha, E(0)\rangle}(1) \mid \alpha \in \mathcal{A}\right\rangle,\left\langle\pi_{\langle\beta, E(0)\rangle,\langle\alpha, E(0)\rangle} \mid \beta \geq_{\mathcal{A}} \alpha \alpha, \beta \in \mathcal{A}\right\rangle\right\rangle .
$$

Note that the difference between $\pi_{\beta, \alpha}$ and $\pi_{\langle\beta, E(0)\rangle,\langle\alpha, E(0)\rangle}$ is quite superficial. We can define $\pi_{\langle\beta, E(0)\rangle,\langle\alpha, E(0)\rangle}$ in a uniform way for both extenders. Just project the first element of the argument using $\pi_{\beta, \alpha}$.

If $\langle E(0), E(1)\rangle \notin M$, then the construction stops. In this case we set

$$
\forall \alpha \in \mathcal{A} \bar{E}_{\alpha}=\langle\alpha, E(0), E(1)\rangle .
$$

We say that $\bar{E}_{\alpha}$ is an extender sequence of length $2\left(1\left(\bar{E}_{\alpha}\right)=2\right)$.

If $\langle E(0), E(1)\rangle \in M$, then we construct the extender $E(2)$ in the same way as we constructed $E(1)$ from $E(0)$.

The above special case being worked out, we continue with the general case. Assume we have constructed

$$
\left\langle E\left(\tau^{\prime}\right) \mid \tau^{\prime}<\tau\right\rangle
$$

If $\left\langle E\left(\tau^{\prime}\right) \mid \tau^{\prime}<\tau\right\rangle \notin M$, then the construction stops here. We set

$$
\forall \alpha \in \mathcal{A} \bar{E}_{\alpha}=\left\langle\alpha, E\left(\tau^{\prime}\right) \mid \tau^{\prime}<\tau\right\rangle,
$$

and we say that $\bar{E}_{\alpha}$ is an extender sequence of length $\tau\left(1\left(\bar{E}_{\alpha}\right)=\tau\right)$.

If, on the other hand, $\left\langle E\left(\tau^{\prime}\right) \mid \tau^{\prime}<\tau\right\rangle \in M$, then we construct

$$
A \in E_{\left\langle\alpha, E(0), \ldots, E\left(\tau^{\prime}\right), \ldots \mid \tau^{\prime}<\tau\right\rangle}(\tau) \Longleftrightarrow\left\langle\alpha, E(0), \ldots, E\left(\tau^{\prime}\right), \ldots \mid \tau^{\prime}<\tau\right\rangle \in j(A) .
$$

Defining $\pi_{\left\langle\beta, E(0), \ldots, E\left(\tau^{\prime}\right), \ldots \mid \tau^{\prime}<\tau\right\rangle,\left\langle\alpha, E(0), \ldots, E\left(\tau^{\prime}\right), \ldots, \mid \tau^{\prime}<\tau\right\rangle}$ using the first coordinate as before gives the needed projection.

We are quite casual in writing the indices of the projections and ultrafilters. By this we mean that we sometimes write $\pi_{\beta, \alpha}$ when we should have written $\pi_{\left\langle\beta, E(0), \ldots, E\left(\tau^{\prime}\right), \ldots \mid \tau^{\prime}<\tau\right\rangle,\left\langle\alpha, E(0), \ldots, E\left(\tau^{\prime}\right), \ldots, \mid \tau^{\prime}<\tau\right\rangle}$ and $E_{\alpha}(\tau)$ when we should have written $E_{\left\langle\alpha, E(0), \ldots, E\left(\tau^{\prime}\right), \ldots, \mid \tau^{\prime}<\tau\right\rangle}(\tau)$.

With this abuse of notation the projection we just defined satisfies

$$
j\left(\pi_{\beta, \alpha}\right)\left(\left\langle\beta, E(0), \ldots, E\left(\tau^{\prime}\right), \ldots \mid \tau^{\prime}<\tau\right\rangle\right)=\left\langle\alpha, E(0), \ldots, E\left(\tau^{\prime}\right), \ldots \mid \tau^{\prime}<\tau\right\rangle,
$$


and we have the extender

$$
E(\tau)=\left\langle\left\langle E_{\alpha}(\tau) \mid \alpha \in \mathcal{A}\right\rangle,\left\langle\pi_{\beta, \alpha} \mid \beta \geq_{\mathcal{A}} \alpha \alpha, \beta \in \mathcal{A}\right\rangle\right\rangle .
$$

We let the construction run until it stops due to the extender sequence not being in $M$.

Definition 2.1. We call $\bar{\mu}$ an extender sequence if there is an elementary embedding $j: V \rightarrow M$ such that $\bar{\nu}$ is an extender sequence generated as above and $\bar{\mu}=\bar{\nu} \mid \tau$ for $\tau \leq 1(\bar{\nu}) . \kappa(\bar{\mu})$ is the ordinal at the beginning of the sequence (i.e., $\kappa\left(\bar{E}_{\alpha}\right)=\alpha$ ), and $\kappa^{0}(\bar{\mu})$ is $(\kappa(\bar{\mu}))^{0}$ (i.e., $\kappa^{0}\left(\bar{E}_{\alpha}\right)=\kappa$ ).

That is, we do not have to construct the extender sequence until it is not in $M$. We can stop anywhere on the way.

The generalization of the measure on the $\alpha$ coordinate in Gitik-Magidor forcing [3] is $\bar{E}_{\alpha}$.

Definition 2.2. A sequence of extender sequences $\left\langle\bar{\mu}_{1}, \ldots, \bar{\mu}_{n}\right\rangle$ is called ${ }^{0}$-increasing if $\kappa^{0}\left(\bar{\mu}_{1}\right)<\cdots<\kappa^{0}\left(\bar{\mu}_{n}\right)$.

Definition 2.3. Let $\left\langle\bar{\mu}_{1}, \ldots, \bar{\mu}_{n}\right\rangle$ be ${ }^{0}$-increasing. An extender sequence $\bar{\mu}$ is called permitted to $\left\langle\bar{\mu}_{1}, \ldots, \bar{\mu}_{n}\right\rangle$ if $\kappa\left(\bar{\mu}_{n}\right)<\kappa^{0}(\bar{\mu})$.

Definition 2.4. We say $A \in \bar{E}_{\alpha}$ if $\forall \xi<\mathrm{l}\left(\bar{E}_{\alpha}\right), A \in E_{\alpha}(\xi)$.

Definition 2.5. $\bar{E}=\left\langle\bar{E}_{\alpha} \mid \alpha \in \mathcal{A}\right\rangle$ is an extender sequence system if there is an elementary embedding $j: V \rightarrow M$ such that all $\bar{E}_{\alpha}$ are extender sequences generated from $j$ as prescribed above and $\forall \alpha, \beta \in \mathcal{A}, \mathrm{l}\left(\bar{E}_{\alpha}\right)=\mathrm{l}\left(\bar{E}_{\beta}\right)$. This common length is called the length of the system, $1(\bar{E})$. We write $\bar{E}(\bar{\mu})$ for the extender sequence system to which $\bar{\mu}$ belongs (i.e., $\left.\bar{E}\left(\bar{E}_{\alpha}\right)=\bar{E}\right)$.

We point out that there is a $\kappa^{+}$-directed partial order on $\bar{E}$ inherited from $\mathcal{A}$. That is, $\bar{E}_{\beta} \geq_{\overline{\mathrm{E}}} \bar{E}_{\alpha} \Longleftrightarrow \beta \geq_{\mathcal{A}} \alpha$. Of course, this implies that there is $\min \bar{E}$, namely $\bar{E}_{\kappa}$. From now on we use only the order $\geq_{\overline{\mathrm{E}}}$, even for $\mathcal{A}$, and we write $\operatorname{dom} \bar{E}$ for $\mathcal{A}$.

\section{2. $\bar{E}_{\gamma}$-tree.}

Definition 2.6. A tree $T$ is an $\bar{E}_{\alpha}$-tree if its elements are of the form

$$
\left\langle\left\langle\bar{\mu}_{1}, \ldots, \bar{\mu}_{n}\right\rangle, S\right\rangle
$$

where

(1) if we set $\operatorname{dom} T=\left\{\left\langle\bar{\mu}_{1}, \ldots, \bar{\mu}_{n}\right\rangle \mid\left\langle\left\langle\bar{\mu}_{1}, \ldots, \bar{\mu}_{n}\right\rangle, S\right\rangle \in T\right\}$, then the function

$$
\left\langle\bar{\mu}_{1}, \ldots, \bar{\mu}_{n}\right\rangle \mapsto\left\langle\left\langle\bar{\mu}_{1}, \ldots, \bar{\mu}_{n}\right\rangle, S\right\rangle
$$

from $\operatorname{dom} T$ to $T$ is $1-1$ and onto,

(2) $t \in \operatorname{Lev}_{n}(\operatorname{dom} T) \Longrightarrow|t|=n+1$,

(3) $\left\langle\bar{\mu}_{1}, \ldots, \bar{\mu}_{n}\right\rangle$ is a ${ }^{0}$-increasing sequence,

(4) $\operatorname{Lev}_{0}(\operatorname{dom} T) \in \bar{E}_{\alpha}$, and for each $t \in \operatorname{dom} T, \operatorname{Suc}_{\operatorname{dom} T}(t) \in \bar{E}_{\alpha}$,

(5) $S$ is a $\bar{\mu}_{n}$-tree. When $\mathrm{l}\left(\bar{\mu}_{n}\right)=0$ we set $S=\emptyset$.

Note that this clause is recursive.

Note 2.7. While we call $T$ a tree, formally speaking, it is a function with domain a tree (in the usual sense) of finite sequences. 
Note 2.8. Later on, we abuse notation and use $T$ instead of $\operatorname{dom} T$. That is, we write $\operatorname{Suc}_{T}(t)$ instead of $\operatorname{Suc}_{\text {dom } T}(t)$.

Definition 2.9. Assume $T$ is a $\bar{E}_{\alpha}$-tree and $t \in T$. Then:

(1) $T_{t}=\{\langle s, S\rangle \mid\langle t \frown s, S\rangle \in T\}$.

(2) $T(\bar{\mu})$ is the tree, $S$, satisfying $\langle\bar{\mu}, S\rangle \in \operatorname{Lev}_{0}(T)$.

(3) $T_{t}(\bar{\mu})$ is the tree, $S$, satisfying $\langle\bar{\mu}, S\rangle \in \operatorname{Suc}_{T}(t)$.

Definition 2.10. Let $T, S$ be $\bar{E}_{\alpha}$-trees, where $\mathrm{l}(\bar{E})=1$. We say that $T \leq S$ if

(1) $\operatorname{Lev}_{0}(T) \subseteq \operatorname{Lev}_{0}(S)$, and

(2) $\forall t \in T \operatorname{Suc}_{T}(t) \subseteq \operatorname{Suc}_{S}(t)$.

Definition 2.11. Let $T, S$ be $\bar{E}_{\alpha}$-trees. We say that $T \leq S$ if

(1) $\operatorname{Lev}_{0}(T) \subseteq \operatorname{Lev}_{0}(S)$,

(2) $\forall t \in T \operatorname{Suc}_{T}(t) \subseteq \operatorname{Suc}_{S}(t)$,

(3) $\forall\langle\bar{\mu}\rangle \in \operatorname{Lev}_{0}(T) T(\bar{\mu}) \leq S(\bar{\mu})$, and

(4) $\forall t \in T \forall\langle\bar{\mu}\rangle \in T_{t} T_{t}(\bar{\mu}) \leq S_{t}(\bar{\mu})$.

Note that the last 2 conditions are recursive.

Definition 2.12. Let $S$ be $\bar{E}_{\alpha}$-tree and $\beta>\alpha$. Define $T=\pi_{\beta, \alpha}^{-1}(S)$ by

(1) $\operatorname{dom} T=\pi_{\beta, \alpha}^{-1}(\operatorname{dom} S)$

(2) $T_{\left\langle\bar{\mu}_{1}, \ldots, \bar{\mu}_{n-1}\right\rangle}\left(\bar{\mu}_{n}\right)=\pi_{\bar{\mu}_{n}, \pi_{\beta, \alpha}\left(\bar{\mu}_{n}\right)}^{-1} S_{\left\langle\pi_{\beta, \alpha}\left(\bar{\mu}_{1}\right), \ldots, \pi_{\beta, \alpha}\left(\bar{\mu}_{n-1}\right)\right\rangle}\left(\pi_{\beta, \alpha}\left(\bar{\mu}_{n}\right)\right)$.

Definition 2.13. Let $T, S$ be $\bar{E}_{\beta}, \bar{E}_{\alpha}$-trees respectively, where $\beta \geq_{\overline{\mathrm{E}}} \alpha$. We say that $T \leq S$ if

(1) $T \leq \pi_{\beta, \alpha}^{-1}(S)$.

Definition 2.14. Assume we have $A_{\langle\bar{\nu}, R\rangle}$, where $\bar{\nu}$ is an extender sequence such that each element in $A_{\langle\bar{\nu}, R\rangle}$ is of the form $\langle\bar{\mu}, S\rangle$ where $\bar{\mu}$ is an extender sequence and $S$ is a tree (in this work $S$ is always a $\bar{\mu}$-tree). We define $\triangle_{\langle\bar{\nu}, R\rangle}^{0} A_{\langle\bar{\nu}, R\rangle}$ as

$$
\langle\bar{\mu}, S\rangle \in \underset{\langle\bar{\nu}, R\rangle}{\triangle^{0}} A_{\langle\bar{\nu}, R\rangle} \Longleftrightarrow \forall\langle\bar{\nu}, R\rangle \kappa(\bar{\nu})<\kappa^{0}(\bar{\mu}) \rightarrow\left\langle\bar{\mu}, S_{\langle\bar{\nu}, R\rangle}\right\rangle \in A_{\langle\bar{\nu}, R\rangle} .
$$

\section{3. $P_{\bar{E}}$-Forcing}

The definition of the forcing is done by induction on $1(\bar{E})$. Originally we started by directly giving the general case. At the suggestion of the referee we have added concrete definitions also for the cases $\mathrm{l}(\bar{E})=1, \mathrm{l}(\bar{E})=2$ and several figures we hope will enhance the intuition standing behind the definitions.

Definition 3.1. Assume $l(\bar{E})=0$. A condition in $P_{\bar{E}}^{*}$ is of the form

$$
\left\{\left\langle\langle\kappa\rangle, p^{\langle\kappa\rangle}\right\rangle\right\}
$$

where $p^{\langle\kappa\rangle} \in V_{\kappa}$ is an extender sequence. Call it $p^{0} .\left(p^{\langle\kappa\rangle}=\emptyset\right.$ is allowed. $)$

When $\mathrm{l}(\bar{E})=0$, the partial order $\leq^{*}$ degenerates into $=$.

Definition 3.2. Assume $\mathrm{l}(\bar{E})=0$. Let $p, q \in P_{\bar{E}}^{*}$. We say that $p$ is a Prikry extension of $q\left(p \leq^{*} q\right.$ or $\left.p \leq^{0} q\right)$ if $p^{0}=q^{0}$.

Definition 3.3. Assume $\mathrm{l}(\bar{E})=0$. We set $\left\langle P_{\bar{E}}, \leq\right\rangle=\left\langle P_{\bar{E}}^{*}, \leq^{*}\right\rangle$. 
Hence, when $\mathrm{l}(\bar{E})=0, P_{\bar{E}}$ is not really a forcing. We use it more as a place-holder in order not to convolute later proofs with two cases everywhere.

Definition 3.4. Assume $\mathrm{l}(\bar{E})=1$. A condition in $P_{\bar{E}}^{*}$ is of the form

$$
\left\{\left\langle\langle\alpha, E(0)\rangle, p^{\langle\alpha, E(0)\rangle}\right\rangle \mid\langle\alpha, E(0)\rangle \in g\right\} \cup\{T\}
$$

where

(1) $g \subseteq \bar{E},|g| \leq \kappa$,

(2) $\min \bar{E}=\langle\kappa, E(0)\rangle \in g$ and $g$ has a maximal element,

(3) $p^{\langle\kappa, E(0)\rangle} \in V_{\kappa^{0}(\bar{E})}$ is an extender sequence which we call $p^{0}$ (we allow $p^{0}=$ $\emptyset)$

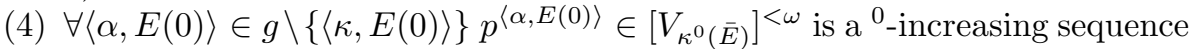
(we allow $p^{\langle\alpha, E(0)\rangle}=\emptyset$ ),

(5) $T$ is a max $g$-tree such that for all $t \in T$, and if $p^{\max g \frown t}$ is ${ }^{0}$-increasing, $\langle\bar{\nu}\rangle \in T$, then $\bar{\nu}=\langle\xi\rangle$ for some $\xi<\kappa$ (meaning that $\bar{\nu}$ is an extender sequence of length 0$)$,

(6) for all $\langle\alpha, E(0)\rangle \in g, p^{0}$ is not permitted to $p^{\langle\alpha, E(0)\rangle \text {, }}$

(7) $\forall\langle\bar{\nu}\rangle \in T \mid\left\{\langle\alpha, E(0)\rangle \in g \mid \bar{\nu}\right.$ is permitted to $\left.p^{\langle\alpha, E(0)\rangle}\right\} \mid \leq \kappa^{0}(\bar{\nu})$, and

(8) $\forall\langle\bar{\nu}\rangle \in T$, if $\bar{\nu}$ is permitted to $p^{\langle\beta, E(0)\rangle}, p^{\langle\alpha, E(0)\rangle}$, then

$$
\pi_{\max g,\langle\beta, E(0)\rangle}(\bar{\nu}) \neq \pi_{\max g,\langle\alpha, E(0)\rangle}(\bar{\nu}) .
$$

We write $\operatorname{mc}(p), p^{\mathrm{mc}}, T^{p}, \bar{E}(p)$, $\operatorname{supp} p$ for $\max g, p^{\max g}, T, \bar{E}, g$ respectively.

Definition 3.5. Assume $\mathrm{l}(\bar{E})=1$. Let $p, q \in P_{\bar{E}}^{*}$. We say that $p$ is a Prikry extension of $q\left(p \leq^{*} q\right.$ or $\left.p \leq^{0} q\right)$ if

(1) $\operatorname{supp} p \supseteq \operatorname{supp} q$,

(2) $\forall\langle\alpha, E(0)\rangle \in \operatorname{supp} q p^{\langle\alpha, E(0)\rangle}=q^{\langle\alpha, E(0)\rangle}$,

(3) $T^{p} \leq \pi_{\operatorname{mc}(p), \operatorname{mc}(q)}^{-1} T^{q}$,

(4) $\forall\langle\alpha, E(0)\rangle \in \operatorname{supp} q \forall\langle\bar{\nu}\rangle \in T^{p}$ $\max \kappa\left(p^{\langle\alpha, E(0)\rangle}\right)<\kappa^{0}(\bar{\nu}) \Longrightarrow \pi_{\mathrm{mc}(p), \alpha}(\bar{\nu})=\pi_{\mathrm{mc}(q), \alpha}\left(\pi_{\mathrm{mc}(p), \operatorname{mc}(q)}(\bar{\nu})\right)$.

Definition 3.6. Assume $l(\bar{E})=1$. A condition in $P_{\bar{E}}$ is of the form

$$
p_{n} \frown \ldots \frown p_{0}
$$

where

- $p_{0} \in P_{E}^{*}$,

- $p_{1} \in P_{\mu_{1}}^{*}$,

- :

- $p_{n} \in P_{\bar{\mu}_{n}}^{*}$,

where $\bar{E}, \bar{\mu}_{1}, \ldots, \bar{\mu}_{n}$ are extender sequence systems such that $1\left(\bar{\mu}_{1}\right)=0, \ldots, \mathrm{l}\left(\bar{\mu}_{n}\right)=$ 0 satisfying

$$
\forall i \leq n-1 \kappa\left(\bar{\mu}_{i+1}\right)<\kappa^{0}\left(\bar{\mu}_{i}\right) \text { where } \bar{\mu}_{0}=\bar{E} .
$$

Definition 3.7. Assume $\mathrm{l}(\bar{E})=1$. Let $p, q \in P_{\bar{E}}$. We say that $p$ is a Prikry extension of $q\left(p \leq^{*} q\right.$ or $\left.p \leq^{0} q\right)$ if $p, q$ are of the form

$$
\begin{aligned}
& p=p_{n} \frown \ldots \frown p_{0}, \\
& q=q_{n} \frown \ldots \frown q_{0},
\end{aligned}
$$

and 
- $p_{0}, q_{0} \in P_{\bar{E}}^{*}, p_{0} \leq^{*} q_{0}$,

- $p_{1}, q_{1} \in P_{\bar{\mu}_{1}}^{*}, p_{1} \leq^{*} q_{1}$,

- :

- $p_{n}, q_{n} \in P_{\bar{\mu}_{n}}^{*}, p_{n} \leq^{*} q_{n}$.

$p_{0\langle\bar{\nu}\rangle}$, defined now, is the basic non-direct extension in $P_{\bar{E}}$ of the condition $p_{0}$, which adds the extender sequence $\bar{\nu} \in T^{p_{0}}$ to the finite sequence.

Definition 3.8. Assume $\mathrm{l}(\bar{E})=1$. Let $p \in P_{\bar{E}}^{*}$ and $\langle\bar{\nu}\rangle \in T^{p}$. In this case $\bar{\nu}=\langle\xi\rangle$ for some $\xi<\kappa$. We define $(p)_{\langle\bar{\nu}\rangle}$ to be $p_{1}^{\prime} \frown p_{0}^{\prime}$ where

(1) $\operatorname{supp} p_{0}^{\prime}=\operatorname{supp} p$,

(2) $\forall\langle\alpha, E(0)\rangle \in \operatorname{supp} p_{0}^{\prime}$,

$$
p_{0}^{\prime\langle\alpha, E(0)\rangle}= \begin{cases}\left\langle\pi_{\mathrm{mc}(p), \alpha}(\xi)\right\rangle, & \max \kappa\left(p^{\langle\alpha, E(0)\rangle}\right)<\kappa^{0}(\xi), \alpha=\kappa, \\ p^{\langle\alpha, E(0)\rangle}-\left\langle\pi_{\mathrm{mc}(p), \alpha}(\xi)\right\rangle, & \max \kappa\left(p^{\langle\alpha, E(0)\rangle}\right)<\kappa^{0}(\xi), \alpha \neq \kappa, \\ p^{\langle\alpha, E(0)\rangle}, & \text { otherwise, }\end{cases}
$$

(3) $T^{p_{0}^{\prime}}=T_{\langle\bar{\nu}\rangle}^{p}$,

(4) $\operatorname{supp} p_{1}^{\prime}=\left\{\left\langle\pi_{\operatorname{mc}(p), \kappa}(\xi)\right\rangle\right\}$,

(5) $p_{1}^{\prime\left\langle\pi_{\mathrm{mc}(p), \kappa}(\xi)\right\rangle}=p^{\langle\kappa, E(0)\rangle}$. (Note that $p_{1}^{\prime} \in P_{\left\langle\pi_{\mathrm{mc}(p), \kappa}\right\rangle}^{*}$ )

Definition 3.9. Assume $\mathrm{l}(\bar{E})=1$. Let $p, q \in P_{\bar{E}}$. We say that $p$ is a 1-point extension of $q\left(p \leq^{1} q\right)$ if $p, q$ are of the form

$$
\begin{aligned}
& p=p_{n+1} \frown p_{n} \frown \ldots \frown p_{0}, \\
& q=q_{n} \frown \ldots \frown q_{0},
\end{aligned}
$$

and

- $p_{i+1}, q_{i} \in P_{\bar{\mu}_{i}}^{*}, p_{i+1} \leq^{*} q_{i}$ for $i=1, \ldots, n$,

- there is $\langle\bar{\nu}\rangle \in T^{q_{0}}$ such that $p_{1} \frown p_{0} \leq^{*}\left(q_{0}\right)_{\langle\bar{\nu}\rangle}$.

Definition 3.10. Assume $\mathrm{l}(\bar{E})=1$. Let $p, q \in P_{\bar{E}}$. We say that $p$ is an $n$-point extension of $q\left(p \leq^{n} q\right)$ if there are $p^{n}, \ldots, p^{0}$ such that

$$
p=p^{n} \leq^{1} \cdots \leq^{1} p^{0}=q .
$$

Definition 3.11. Assume $\mathrm{l}(\bar{E})=1$. Let $p, q \in P_{\bar{E}}$. We say that $p$ is an extension of $q(p \leq q)$ if there is an $n$ such that $p \leq^{n} q$.

It is quite clear that when $1(\bar{E})=1,\left\langle P_{\bar{E}}, \leq\right\rangle$ is the Gitik-Magidor forcing from 3. with the following slight changes:

(1) The support elements are $\langle\alpha, E(0)\rangle$ instead of $\alpha$.

(2) $p^{\langle\kappa, E(0)\rangle}$ has at most one element and not a finite sequence.

We give some figures in order to enhance the intuition behind these definitions. In Figure 1 we show a typical condition $p_{0} \in P_{\bar{E}}^{*}$. In Figure 2 we show a 1-point extension of $p_{0}$ using $\langle\langle\nu\rangle\rangle \in T^{p_{0}}$, assuming $\xi_{1,2}^{0} \geq \nu^{0}$. In Figure 3 we show a 1-point extension of $p_{0\langle\bar{\nu}\rangle}$ using $\langle\langle\mu\rangle\rangle \in T_{\langle\bar{\nu}\rangle}^{p_{0}}$, again assuming $\xi_{2,1} \geq \mu^{0}$.

If $G$ is $P_{\bar{E}}$-generic and we set $C^{\alpha}=\bigcup\left\{p^{\langle\alpha, E(0)\rangle} \mid p \in G,\langle\alpha, E(0)\rangle \in \operatorname{supp} p\right\}$, then $C^{\alpha}$ is an $\omega$-sequence unbounded in $\kappa$.

We continue with the definition of $P_{\bar{E}}$ for $l(\bar{E})=2$. 


\begin{tabular}{cccc} 
& $\left\langle\xi_{1,2}\right\rangle$ & & \\
& $\left\langle\xi_{1,1}\right\rangle$ & $\left\langle\xi_{2,1}\right\rangle$ & \\
$\left\langle\xi_{0,0}\right\rangle$ & $\left\langle\xi_{1,0}\right\rangle$ & $\left\langle\xi_{2,0}\right\rangle$ & $T^{p_{0}}$ \\
\hline$\langle\kappa, E(0)\rangle$ & $\left\langle\alpha_{1}, E(0)\right\rangle$ & $\left\langle\alpha_{2}, E(0)\right\rangle$ &
\end{tabular}

Figure 1. An example of $p_{0} \in P_{\bar{E}}^{*}, \mathrm{l}(\bar{E})=1$

$\frac{\left\langle\xi_{0,0}\right\rangle}{\left\langle\nu^{0}\right\rangle}$

\begin{tabular}{cccc} 
& $\left\langle\xi_{1,2}\right\rangle$ & $\langle\nu\rangle$ & \\
& $\left\langle\xi_{1,1}\right\rangle$ & $\left\langle\xi_{2,1}\right\rangle$ & \\
$\left\langle\nu^{0}\right\rangle$ & $\left\langle\xi_{1,0}\right\rangle$ & $\left\langle\xi_{2,0}\right\rangle$ & $T_{\langle\langle\nu\rangle\rangle}^{p_{0}}$ \\
\hline$\langle\kappa, E(0)\rangle$ & $\left\langle\alpha_{1}, E(0)\right\rangle$ & $\left\langle\alpha_{2}, E(0)\right\rangle$ &
\end{tabular}

FigURE 2. $p_{0\langle\langle\nu\rangle\rangle}$, a 1-point extension of $p_{0}$ using $\langle\nu\rangle$

$\frac{\left\langle\xi_{0,0}\right\rangle}{\left\langle\nu^{0}\right\rangle}$

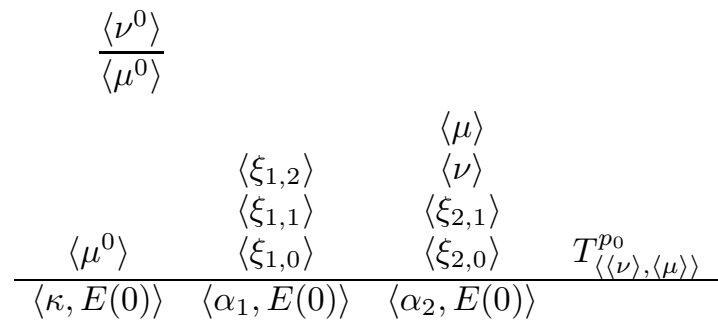

FigURE 3. $p_{0\langle\langle\nu\rangle,\langle\mu\rangle\rangle}$, a 1-point extension of $p_{0\langle\langle\nu\rangle\rangle}$ using $\langle\mu\rangle$

Definition 3.12. Assume $\mathrm{l}(\bar{E})=2$. A condition in $P_{\bar{E}}^{*}$ is of the form

$$
\left\{\left\langle\langle\alpha, E(0), E(1)\rangle, p^{\langle\alpha, E(0), E(1)\rangle}\right\rangle \mid\langle\alpha, E(0), E(1)\rangle \in g\right\} \cup\{T\}
$$

where

(1) $g \subseteq \bar{E},|g| \leq \kappa$,

(2) $\min \bar{E}=\langle\kappa, E(0), E(1)\rangle \in g$ and $g$ has a maximal element,

(3) $p^{\langle\kappa, E(0), E(1)\rangle} \in V_{\kappa^{0}(\bar{E})}$ is an extender sequence that we call $p^{0}$ (we allow $\left.p^{0}=\emptyset\right)$,

(4) $\forall\langle\alpha, E(0), E(1)\rangle \in g \backslash\{\langle\kappa, E(0), E(1)\rangle\} p^{\langle\alpha, E(0), E(1)\rangle} \in\left[V_{\kappa^{0}(\bar{E})}\right]^{<\omega}$ is a ${ }^{0}$-increasing sequence (we allow $p^{\langle\alpha, E(0), E(1)\rangle}=\emptyset$ ),

(5) $T$ is a max $g$-tree such that for all $t \in T, p^{\max g}-t$ is ${ }^{0}$-increasing, and if $\langle\bar{\nu}\rangle \in T$, then either $\bar{\nu}=\langle\xi\rangle$ or $\bar{\nu}=\langle\xi, e(0)\rangle$, where $e(0)$ is extender and $\xi \in \operatorname{dom} e(0)$,

(6) for all $\langle\alpha, E(0), E(1)\rangle \in g, p^{0}$ is not permitted to $p^{\langle\alpha, E(0), E(1)\rangle}$,

(7) $\forall\langle\bar{\nu}\rangle \in T \mid\left\{\langle\alpha, E(0), E(1)\rangle \in g \mid \bar{\nu}\right.$ is permitted to $\left.p^{\langle\alpha, E(0), E(1)\rangle}\right\} \mid \leq \kappa^{0}(\bar{\nu})$, 
(8) $\forall\langle\bar{\nu}\rangle \in T$, if $\bar{\nu}$ is permitted to $p^{\langle\beta, E(0), E(1)\rangle}, p^{\langle\alpha, E(0), E(1)\rangle}$, then

$$
\pi_{\max g,\langle\beta, E(0), E(1)\rangle}(\bar{\nu}) \neq \pi_{\max g,\langle\alpha, E(0), E(1)\rangle}(\bar{\nu}) .
$$

We write $\operatorname{mc}(p), p^{\mathrm{mc}}, T^{p}, \bar{E}(p)$, supp $p$ for $\max g, p^{\max g}, T, \bar{E}, g$ respectively.

Definition 3.13. Assume $\mathrm{l}(\bar{E})=2$. Let $p, q \in P_{\bar{E}}^{*}$. We say that $p$ is a Prikry extension of $q\left(p \leq^{*} q\right.$ or $\left.p \leq^{0} q\right)$ if

(1) $\operatorname{supp} p \supseteq \operatorname{supp} q$,

(2) $\forall\langle\alpha, E(0), E(1)\rangle \in \operatorname{supp} q p^{\langle\alpha, E(0), E(1)\rangle}=q^{\langle\alpha, E(0), E(1)\rangle}$,

(3) $T^{p} \leq \pi_{\operatorname{mc}(p), \operatorname{mc}(q)}^{-1} T^{q}$,

(4) $\forall\langle\alpha, E(0), E(1)\rangle \in \operatorname{supp} q \forall\langle\bar{\nu}\rangle \in T^{p}$

$$
\max \kappa\left(p^{\langle\alpha, E(0), E(1)\rangle}\right)<\kappa^{0}(\bar{\nu}) \Longrightarrow \pi_{\operatorname{mc}(p), \alpha}(\bar{\nu})=\pi_{\operatorname{mc}(q), \alpha}\left(\pi_{\operatorname{mc}(p), \operatorname{mc}(q)}(\bar{\nu})\right) .
$$

Definition 3.14. Assume $\mathrm{l}(\bar{E})=2$. A condition in $P_{\bar{E}}$ is of the form

$$
p_{n} \frown \ldots \frown p_{0}
$$

where

- $p_{0} \in P_{\bar{E}}^{*}$,

- $p_{1} \in P_{\bar{\mu}_{1}}^{*}$,

- :

- $p_{n} \in P_{\bar{\mu}_{n}}^{*}$,

where $\bar{E}, \bar{\mu}_{1}, \ldots, \bar{\mu}_{n}$ are extender sequence systems such that $\mathrm{l}\left(\bar{\mu}_{1}\right)<2, \ldots, \mathrm{l}\left(\bar{\mu}_{n}\right)<$ 2 satisfying

$$
\forall i \leq n-1 \kappa\left(\bar{\mu}_{i+1}\right)<\kappa^{0}\left(\bar{\mu}_{i}\right) \text { where } \bar{\mu}_{0}=\bar{E} .
$$

Definition 3.15. Assume $\mathrm{l}(\bar{E})=2$. Let $p, q \in P_{\bar{E}}$. We say that $p$ is a Prikry extension of $q\left(p \leq^{*} q\right.$ or $\left.p \leq^{0} q\right)$ if $p, q$ are of the form

$$
\begin{aligned}
& p=p_{n} \frown \ldots \frown p_{0}, \\
& q=q_{n} \frown \ldots \frown q_{0},
\end{aligned}
$$

and

- $p_{0}, q_{0} \in P_{\bar{E}}^{*}, p_{0} \leq^{*} q_{0}$,

- $p_{1}, q_{1} \in P_{\bar{\mu}_{1}}^{*}, p_{1} \leq^{*} q_{1}$

- :

- $p_{n}, q_{n} \in P_{\bar{\mu}_{n}}^{*}, p_{n} \leq^{*} q_{n}$.

$p_{0\langle\bar{\nu}\rangle}$, defined now, is the basic non-direct extension in $P_{\bar{E}}$ of the condition $p_{0}$, which adds the extender sequence $\bar{\nu} \in T^{p_{0}}$ to the finite sequence. If $\bar{\nu}=\langle\xi, e(0)\rangle$, then a condition $p_{1}^{\prime} \in P_{e(0)}$ is added.

Definition 3.16. Assume $\mathrm{l}(\bar{E})=2$. Let $p \in P_{\bar{E}}^{*}$ and $\langle\bar{\nu}\rangle \in T^{p}$. We define $p_{\langle\bar{\nu}\rangle}$ to be $p_{1}^{\prime} \frown p_{0}^{\prime}$, where

(1) $\operatorname{supp} p_{0}^{\prime}=\operatorname{supp} p$, 
(2) $\forall\langle\alpha, E(0), E(1)\rangle \in \operatorname{supp} p_{0}^{\prime} p_{0}^{\prime\langle\alpha, E(0), E(1)\rangle}=$

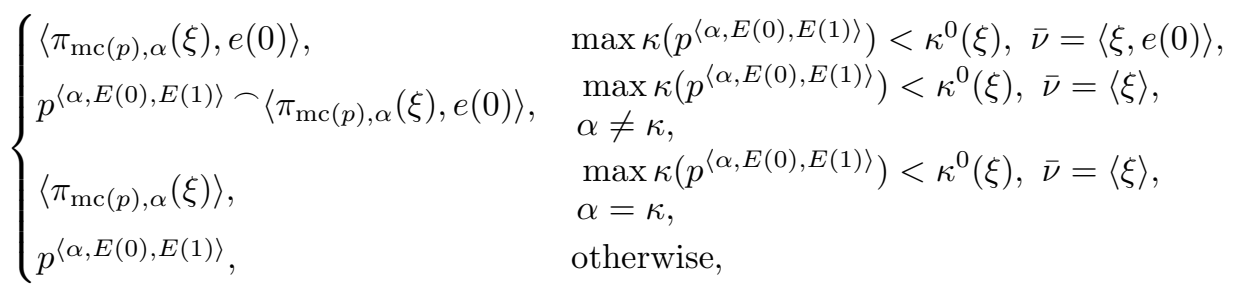

(3) $T^{p_{0}^{\prime}}=T_{\langle\bar{\nu}\rangle}^{p}$,

(4) if $\bar{\nu}=\langle\xi\rangle$, then

$$
\begin{aligned}
& \text { (4.1) } \operatorname{supp} p_{1}^{\prime}=\left\{\left\langle\pi_{\operatorname{mc}(p), \kappa}(\xi)\right\rangle\right\}, \\
& \text { (4.2) } p_{1}^{\prime\left\langle\pi_{\operatorname{mc}(p), \kappa}(\xi)\right\rangle}=p^{\langle\kappa, E(0), E(1)\rangle}, \\
& \text { (4.3) } T^{p_{1}^{\prime}}=\emptyset,
\end{aligned}
$$

(5) if $\bar{\nu}=\langle\xi, e(0)\rangle$, then

(5.1) $\operatorname{supp} p_{1}^{\prime}=\left\{\left\langle\pi_{\mathrm{mc}(p), \alpha}(\xi), e(0)\right\rangle \mid\langle\alpha, E(0), E(1)\rangle \in \operatorname{supp} p\right.$, $\left.\max \kappa\left(p^{\langle\alpha, E(0), E(1)\rangle}\right)<\kappa^{0}(\xi)\right\}$

(5.2) $\forall\left\langle\pi_{\mathrm{mc}(p), \alpha}(\xi), e(0)\right\rangle \in \operatorname{supp} p_{1}^{\prime} p_{1}^{\prime\left\langle\pi_{\mathrm{mc}(p), \alpha}(\xi), e(0)\right\rangle}=p^{\langle\alpha, E(0), E(1)\rangle}$,

(5.3) $T^{p_{1}^{\prime}}=T^{p}(\langle\xi, e(0)\rangle)$ (note that $\left.p_{1}^{\prime} \in P_{e(0)}^{*}\right)$.

Definition 3.17. Assume $1(\bar{E})=2$. Let $p, q \in P_{\bar{E}}$. We say that $p$ is a 1-point extension of $q\left(p \leq{ }^{1} q\right)$ if $p, q$ are of the form

$$
\begin{aligned}
& p=p_{n+1} \frown p_{n} \frown \ldots \frown p_{0}, \\
& q=q_{n} \frown \ldots \frown q_{0},
\end{aligned}
$$

and there is $0 \leq k \leq n$ such that

- $p_{i}, q_{i} \in P_{\bar{\mu}_{i}}^{*}, p_{i} \leq^{*} q_{i}$ for $i=0, \ldots, k-1$,

- $p_{i+1}, q_{i} \in P_{\bar{\mu}_{i}}^{*}, p_{i+1} \leq^{*} q_{i}$ for $i=k+1, \ldots, n$,

- there is $\langle\bar{\nu}\rangle \in T^{q_{k}}$ such that $p_{k+1} \frown p_{k} \leq^{*}\left(q_{k}\right)_{\langle\bar{\nu}\rangle}$.

Definition 3.18. Assume $\mathrm{l}(\bar{E})=2$. Let $p, q \in P_{\bar{E}}$. We say that $p$ is an $n$-point extension of $q\left(p \leq^{n} q\right)$ if there are $p^{n}, \ldots, p^{0}$ such that

$$
p=p^{n} \leq^{1} \cdots \leq^{1} p^{0}=q .
$$

Definition 3.19. Assume $\mathrm{l}(\bar{E})=2$. Let $p, q \in P_{\bar{E}}$. We say that $p$ is an extension of $q(p \leq q)$ if there is an $n$ such that $p \leq^{n} q$.

When $\mathrm{l}(\bar{E})=2$, the forcing $P_{\bar{E}}$ is similar to a special case of the forcing defined in 8. Again we give some figures in order to enhance the intuition behind these definitions. In Figure 4 we show a typical condition $p_{0} \in P_{\bar{E}}^{*}$. In Figure 5 we show a 1-point extension of $p_{0}$ using $\langle\langle\nu\rangle\rangle \in T^{p_{0}}$, assuming $\xi_{1,2}^{0} \geq \nu^{0}$. In Figure 6 we show a 1-point extension of $p_{0\langle\bar{\nu}\rangle}$ using $\langle\langle\mu\rangle\rangle \in T_{\langle\bar{\nu}\rangle}^{p_{0}}$, again assuming $\xi_{2,1} \geq \mu^{0}$.

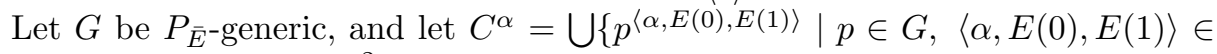
$\operatorname{supp} p$ \}. Then $C^{\alpha}$ is an $\omega^{2}$-sequence unbounded in $\kappa$.

Set also $C^{\mu}=\bigcup\left\{p^{\langle\mu, e(0)\rangle} \mid p \in G,\langle\mu, e(0)\rangle \in \operatorname{supp} p\right\}$. Then $C^{\mu}$ is an $\omega$-sequence unbounded in $\nu^{0}$.

We now leave the special cases and define the forcing notion for arbitrary $\mathrm{l}(\bar{E})$. 


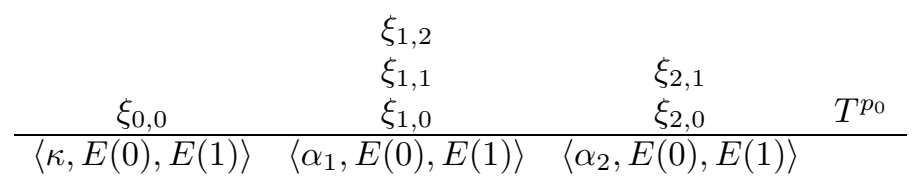

Figure 4. An example of $p_{0} \in P_{\bar{E}}^{*}, \mathrm{l}(\bar{E})=2$

$\frac{\xi_{0,0}}{\left\langle\nu^{0}\right\rangle}$

\begin{tabular}{cccc} 
& $\xi_{1,2}$ & $\nu$ & \\
& $\xi_{1,1}$ & $\xi_{2,1}$ & \\
$\nu^{0}$ & $\xi_{1,0}$ & $\xi_{2,0}$ & $T_{\langle\nu\rangle}^{p_{0}}$ \\
\hline$\langle\kappa, E(0), E(1)\rangle$ & $\left\langle\alpha_{1}, E(0), E(1)\right\rangle$ & $\left\langle\alpha_{2}, E(0), E(1)\right\rangle$ &
\end{tabular}

FIGURE 5. $p_{0\langle\bar{\nu}\rangle}$, a 1-point extension of $p_{0}$ using $\langle\nu\rangle$

$\frac{\xi_{0,0}}{\left\langle\nu^{0}\right\rangle}$

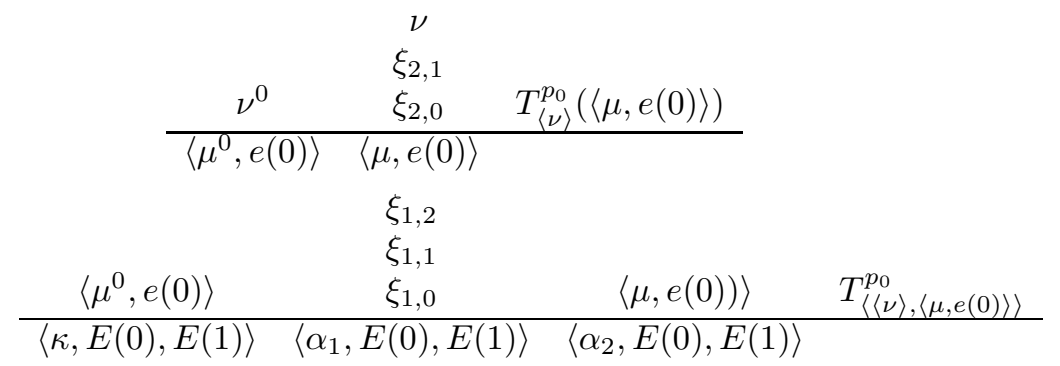

Figure $6 . p_{0\langle\nu,\langle\mu, e(0)\rangle\rangle}$, a 1-point extension of $p_{0\langle\nu\rangle}$ using $\langle\mu, e(0)\rangle$

Definition 3.20. A condition in $P_{\bar{E}}^{*}$ is of the form

$$
\left\{\left\langle\bar{\gamma}, p^{\bar{\gamma}}\right\rangle \mid \bar{\gamma} \in g\right\} \cup\{T\}
$$

where

(1) $g \subseteq \bar{E},|g| \leq \kappa$,

(2) $\min \bar{E}=\bar{E}_{\kappa} \in g$ and $g$ has a maximal element,

(3) $p^{\min \bar{E}} \in V_{\kappa^{0}(\bar{E})}$ is an extender sequence, call it $p^{0}$ (we allow $p^{0}=\emptyset$ ),

(4) $\forall \bar{\gamma} \in g \backslash\{\min \bar{E}\} p^{\bar{\gamma}} \in\left[V_{\kappa^{0}(\bar{E})}\right]^{<\omega}$ is a ${ }^{0}$-increasing sequence (we allow $\left.p^{\bar{\gamma}}=\emptyset\right)$

(5) $T$ is a max $g$-tree such that for all $t \in T, p^{\max g \frown t} t$ is ${ }^{0}$-increasing,

(6) for all $\bar{\gamma} \in g, p^{0}$ is not permitted to $p^{\bar{\gamma}}$,

(7) $\forall\langle\bar{\nu}\rangle \in T \mid\left\{\bar{\gamma} \in g \mid \bar{\nu}\right.$ is permitted to $\left.p^{\bar{\gamma}}\right\} \mid \leq \kappa^{0}(\bar{\nu})$,

(8) $\forall\langle\bar{\nu}\rangle \in T$ if $\bar{\nu}$ is permitted to $p^{\bar{\beta}}, p^{\bar{\gamma}}$, then $\pi_{\max g, \bar{\beta}}(\bar{\nu}) \neq \pi_{\max g, \bar{\gamma}}(\bar{\nu})$.

We write $\operatorname{mc}(p), p^{\mathrm{mc}}, T^{p}, \bar{E}(p)$, supp $p$ for $\max g, p^{\max g}, T, \bar{E}, g$ respectively. 
Definition 3.21. Let $p, q \in P_{\bar{E}}^{*}$. We say that $p$ is a Prikry extension of $q\left(p \leq^{*} q\right.$ or $\left.p \leq{ }^{0} q\right)$ if

(1) $\operatorname{supp} p \supseteq \operatorname{supp} q$,

(2) $\forall \gamma \in \operatorname{supp} q p^{\gamma}=q^{\gamma}$,

(3) $T^{p} \leq \pi_{\operatorname{mc}(p), \operatorname{mc}(q)}^{-1} T^{q}$,

(4) $\forall \bar{\gamma} \in \operatorname{supp} q \forall\langle\bar{\nu}\rangle \in T^{p}$

$$
\max \kappa\left(p^{\bar{\gamma}}\right)<\kappa^{0}(\bar{\nu}) \Longrightarrow \pi_{\mathrm{mc}(p), \bar{\gamma}}(\bar{\nu})=\pi_{\mathrm{mc}(q), \bar{\gamma}}\left(\pi_{\mathrm{mc}(p), \operatorname{mc}(q)}(\bar{\nu})\right) .
$$

Note that if $p, q \in P_{\bar{E}}$ satisfy all details of the above definition except 4 then by just shrinking $T^{p}$ we can get 4 also. The proof is the same as the one given in 3 .

In many cases in the sequel we use $\pi_{\bar{\beta}, \bar{\alpha}}^{-1} T$. Whenever we do so we automatically assume the above shrinkage, which of course depends on the conditions used.

Definition 3.22. A condition in $P_{\bar{E}}$ is of the form

$$
p_{n} \frown \ldots \frown p_{0}
$$

where

- $p_{0} \in P_{E}^{*}$,

- $p_{1} \in P_{\bar{\mu}_{1}}^{*}$,

- :

- $p_{n} \in P_{\bar{\mu}_{n}}^{*}$,

where $\bar{E}, \bar{\mu}_{1}, \ldots, \bar{\mu}_{n}$ are extender sequence systems such that $1\left(\bar{\mu}_{1}\right)<\mathrm{l}(\bar{E}), \ldots$, $\mathrm{l}\left(\bar{\mu}_{n}\right)<\mathrm{l}(\bar{E})$ satisfying

$$
\forall i \leq n-1 \kappa\left(\bar{\mu}_{i+1}\right)<\kappa^{0}\left(\bar{\mu}_{i}\right) \text { where } \bar{\mu}_{0}=\bar{E} .
$$

Definition 3.23. Let $p, q \in P_{\bar{E}}$. We say that $p$ is a Prikry extension of $q\left(p \leq^{*} q\right.$ or $p \leq{ }^{0} q$ ) if $p, q$ are of the form

$$
\begin{aligned}
& p=p_{n} \frown \ldots \frown p_{0}, \\
& q=q_{n} \frown \ldots \frown q_{0},
\end{aligned}
$$

and

- $p_{0}, q_{0} \in P_{E}^{*}, p_{0} \leq^{*} q_{0}$,

- $p_{1}, q_{1} \in P_{\bar{\mu}_{1}}^{*}, p_{1} \leq^{*} q_{1}$,

- :

- $p_{n}, q_{n} \in P_{\bar{\mu}_{n}}^{*}, p_{n} \leq^{*} q_{n}$.

$p_{0\langle\bar{\nu}\rangle}$, defined now, is the basic non-direct extension in $P_{\bar{E}}$ of the condition $p_{0}$, which adds the extender sequence $\bar{\nu} \in T^{p_{0}}$ to the finite sequence. A condition $p_{1}^{\prime} \in P_{\bar{E}(\bar{\nu})}$ is added.

Definition 3.24. Let $p \in P_{\bar{E}}^{*}$ and $\langle\bar{\nu}\rangle \in T^{p}$. We define $(p)_{\langle\bar{\nu}\rangle}$ to be $p_{1}^{\prime} \frown p_{0}^{\prime}$ where

(1) $\operatorname{supp} p_{0}^{\prime}=\operatorname{supp} p$,

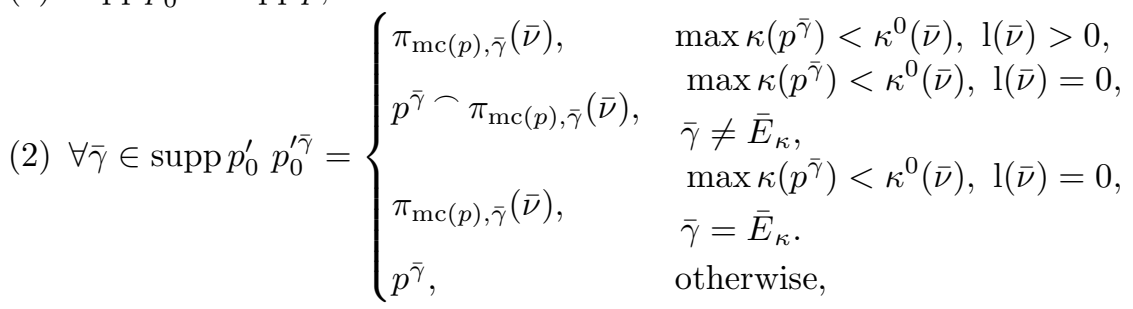


(3) $T^{p_{0}^{\prime}}=T_{\langle\bar{\nu}\rangle}^{p}$,

(4) if $\mathrm{l}(\bar{\nu})=0$, then

(4.1) $\operatorname{supp} p_{1}^{\prime}=\left\{\pi_{\mathrm{mc}(p), \bar{E}_{\kappa}}(\bar{\nu})\right\}$,

(4.2) $p_{1}^{\prime \pi_{\mathrm{mc}(p), \bar{E}_{\kappa}}(\bar{\nu})}=p^{\bar{E}_{\kappa}}$,

(4.3) $T^{p_{1}^{\prime}}=\emptyset$

(5) if $\mathrm{l}(\bar{\nu})>0$, then

(5.1) $\operatorname{supp} p_{1}^{\prime}=\left\{\pi_{\operatorname{mc}(p), \bar{\gamma}}(\bar{\nu}) \mid \bar{\gamma} \in \operatorname{supp} p, \max \kappa\left(p^{\bar{\gamma}}\right)<\kappa^{0}(\bar{\nu})\right\}$,

(5.2) $\forall \pi_{\mathrm{mc}(p), \bar{\gamma}}(\bar{\nu}) \in \operatorname{supp} p_{1}^{\prime} p_{1}^{\prime \pi_{\mathrm{mc}(p), \bar{\gamma}(\bar{\nu})}}=p^{\bar{\gamma}}$,

(5.3) $T^{p_{1}^{\prime}}=T^{p}(\bar{\nu})$ (note that $p_{1}^{\prime} \in P_{\bar{\epsilon}}^{*}$ for $\bar{\epsilon}$ associated with $\bar{\nu}$ ).

Definition 3.25. Let $p, q \in P_{\bar{E}}$. We say that $p$ is a 1-point extension of $q\left(p \leq^{1} q\right)$ if $p, q$ are of the form

$$
\begin{aligned}
& p=p_{n+1} \frown p_{n} \frown \ldots \frown p_{0}, \\
& q=q_{n} \frown \ldots \frown q_{0},
\end{aligned}
$$

and there is $0 \leq k \leq n$ such that

- $p_{i}, q_{i} \in P_{\bar{\mu}_{i}}^{*}, p_{i} \leq^{*} q_{i}$ for $i=0, \ldots, k-1$,

- $p_{i+1}, q_{i} \in P_{\bar{\mu}_{i}}^{*}, p_{i+1} \leq^{*} q_{i}$ for $i=k+1, \ldots, n$,

- there is $\langle\bar{\nu}\rangle \in T^{q_{k}}$ such that $p_{k+1} \frown p_{k} \leq^{*}\left(q_{k}\right)_{\langle\bar{\nu}\rangle}$.

Definition 3.26. Let $p, q \in P_{\bar{E}}$. We say that $p$ is an $n$-point extension of $q\left(p \leq^{n} q\right)$ if there are $p^{n}, \ldots, p^{0}$ such that

$$
p=p^{n} \leq^{1} \cdots \leq^{1} p^{0}=q .
$$

Definition 3.27. Let $p, q \in P_{\bar{E}}$. We say that $p$ is an extension of $q(p \leq q)$ if there is an $n$ such that $p \leq^{n} q$.

Later on by $P_{\bar{E}}$ we mean $\left\langle P_{\bar{E}}, \leq\right\rangle$.

$$
\begin{array}{cccc} 
& \bar{\xi}_{1,2} & & \\
& \bar{\xi}_{1,1} & \bar{\xi}_{2,1} & \\
\bar{\xi}_{0,0} & \bar{\xi}_{1,0} & \bar{\xi}_{2,0} & T^{p_{0}} \\
\hline \bar{E}_{\kappa} & \bar{E}_{\alpha_{1}} & \bar{E}_{\alpha_{2}} &
\end{array}
$$

Figure 7. An example of $p_{0} \in P_{\bar{E}}^{*}$

Note 3.28. When $\mathrm{l}(\bar{E})=1$ the forcing $P_{\bar{E}}$ is the Gitik-Magidor forcing from section 1 of [3]. When $\mathrm{l}(\bar{E})<\kappa$ the forcing $P_{\bar{E}}$ is similar to the forcing defined in [8].

In several places we want to prevent enlargement of the support of a condition. This makes all the conditions which are stronger than some condition but with the same support resemble Radin forcing. The following definition catches the meaning of not enlarging the support. The 'resemblance' we look for is 5.3

Definition 3.29. Let $p, q \in P_{\bar{E}}^{*}$. We say that $p \leq_{R}^{*} q$ if

(1) $p \leq^{*} q$, and

(2) $\operatorname{supp} p=\operatorname{supp} q$. 


\begin{tabular}{cccc} 
& $\bar{\xi}_{1,2}$ & $\langle\nu\rangle$ & \\
& $\bar{\xi}_{1,1}$ & $\bar{\xi}_{2,1}$ & \\
$\left\langle\nu^{0}\right\rangle$ & $\bar{\xi}_{1,0}$ & $\bar{\xi}_{2,0}$ & $T_{\langle\langle\nu\rangle\rangle}^{p_{0}}$ \\
\hline $\bar{E}_{\kappa}$ & $\bar{E}_{\alpha_{1}}$ & $\bar{E}_{\alpha_{2}}$ &
\end{tabular}

FIGURE 8. $p_{0\langle\bar{\nu}\rangle}$, a 1-point extension of $p_{0}$ using $\langle\nu\rangle$

$\frac{\bar{\xi}_{0,0}}{\left\langle\nu^{0}\right\rangle}$

\begin{tabular}{ccc} 
& $\langle\nu\rangle$ & \\
& $\bar{\xi}_{2,1}$ & \\
$\left\langle\nu^{0}\right\rangle$ & $\bar{\xi}_{2,0}$ & $T_{\langle\langle\nu\rangle\rangle}^{p_{0}}(\bar{\mu})$ \\
\hline $\bar{\mu}^{0}$ & $\bar{\mu}$ &
\end{tabular}

\begin{tabular}{cccc} 
& $\bar{\xi}_{1,2}$ & & \\
& $\bar{\xi}_{1,1}$ & & \\
$\bar{\mu}^{0}$ & $\bar{\xi}_{1,0}$ & $\bar{\mu}$ & $T_{\langle\langle\nu\rangle, \bar{\mu}\rangle}^{p_{0}}$ \\
\hline $\bar{E}_{\kappa}$ & $\bar{E}_{\alpha_{1}}$ & $\bar{E}_{\alpha_{2}}$ &
\end{tabular}

FigURE 9. $p_{0\langle\langle\nu\rangle, \bar{\mu}\rangle}$, a 1-point extension of $p_{0\langle\nu\rangle}$ using $\bar{\mu}$

Definition 3.30. Let $p, q \in P_{\bar{E}}$. We say that $p \leq_{R}^{1} q$ if

(1) $p \leq^{1} q$, and

(2) in the definition of $\leq^{1}$, we can replace $\leq^{*}$ by $\leq_{R}^{*}$.

Definition 3.31. Let $p, q \in P_{\bar{E}}$. We say that $p \leq_{R}^{n} q$ if there are $p^{n}, \ldots, p^{0}$ such that

$$
p=p^{n} \leq_{R}^{1} \cdots \leq_{R}^{1} p^{0}=q .
$$

Definition 3.32. Let $p, q \in P_{\bar{E}}$. We say that $p \leq_{R} q$ if there is an $n$ such that $p \leq_{R}^{n} q$.

Note 3.33. The above definitions imply that if $p \leq q$, then there is an $r$ such that $p \leq{ }^{*} r \leq_{R} q$.

Note 3.34. When $\mathrm{l}(\bar{E})=1$ and we force with $\left\langle P_{\bar{E}}, \leq_{R}\right\rangle$ below some $p$ with maximal coordinate $\alpha$, we are forcing just the tree Prikry forcing for the measure $E_{\alpha}(0)$.

Definition 3.35. Let $\bar{\epsilon}$ be an extender sequence such that $\kappa^{0}(\bar{\epsilon})<\kappa^{0}(\bar{E})$. Then

$$
P_{\bar{E}} / P_{\bar{\epsilon}}=\left\{p \mid \exists q \in P_{\bar{\epsilon}}, q^{\frown} p \in P_{\bar{E}}\right\} .
$$

\section{RAdin Forcing}

The main aims of this section are 4.8, 4.10 Since the simplest way we found to formulate them was with Radin forcing [7], [5], [10], we took the opportunity to 
depart from the usual formulation in order to work in the spirit of the extenderbased forcing we defined in section 3 .

The main point is that possible extensions of a condition are stored in an $\bar{E}_{\alpha^{-}}$ tree and not in a set. The $\alpha$ is fixed; so practically we deal here with a measure sequence and not an extender sequence.

Definition 4.1. A condition in $R_{\alpha}$ is of the form

$$
\left\langle\left\langle\bar{\mu}_{n}, s^{n}\right\rangle, S^{n}, \ldots,\left\langle\bar{\mu}_{1}, s^{1}\right\rangle, S^{1},\left\langle\bar{\mu}_{0}, s^{0}\right\rangle, S^{0}\right\rangle,
$$

where

(1) $\bar{\mu}_{0}, \ldots, \bar{\mu}_{n}$ are extender sequences,

(2) $\forall i \leq n-1 \kappa\left(\bar{\mu}_{i+1}\right)<\kappa^{0}\left(\bar{\mu}_{i}\right)$,

(3) $\bar{\mu}_{0}=\bar{E}_{\alpha}$

(4) $\forall i \leq n S^{i}$ is a $\bar{\mu}_{i}$-tree,

(5) $\forall i \leq n s^{i} \in V_{\kappa^{0}\left(\bar{\mu}_{i}\right)}$ is an extender sequence.

Definition 4.2. Let $p, q \in R_{\alpha}$. We say that $p$ is a Prikry extension of $q\left(p \leq^{*} q\right.$ or $\left.p \leq{ }^{0} q\right)$ if $p, q$ are of the form

$$
\begin{aligned}
& p=\left\langle\left\langle\bar{\mu}_{n}, s^{n}\right\rangle, S^{n}, \ldots,\left\langle\bar{\mu}_{1}, s^{1}\right\rangle, S^{1},\left\langle\bar{E}_{\alpha}, s^{0}\right\rangle, S^{0}\right\rangle, \\
& q=\left\langle\left\langle\bar{\mu}_{n}, t^{n}\right\rangle, T^{n}, \ldots,\left\langle\bar{\mu}_{1}, t^{1}\right\rangle, T^{1},\left\langle\bar{E}_{\alpha}, t^{0}\right\rangle, T^{0}\right\rangle,
\end{aligned}
$$

and

- $\forall i \leq n S^{i} \leq T^{i}$,

- $\forall i \leq n s^{i}=t^{i}$.

Definition 4.3. Let $p=\left\langle\left\langle\bar{\mu}_{n}, s^{n}\right\rangle, S^{n}, \ldots,\left\langle\bar{\mu}_{1}, s^{1}\right\rangle, S^{1},\left\langle\bar{\mu}_{0}, s^{0}\right\rangle, S^{0}\right\rangle$ where $\bar{\mu}_{0}=\bar{E}_{\alpha}$. Let $\langle\bar{\nu}\rangle \in S^{i}$. We define $(p)_{\langle\bar{\nu}\rangle}$ to be

$$
\begin{array}{r}
(p)_{\langle\bar{\nu}\rangle}=\left\langle\left\langle\bar{\mu}_{n}, s^{n}\right\rangle, S^{n}, \ldots,\left\langle\bar{\mu}_{i+1}, s^{i+1}\right\rangle, S^{i+1},\right. \\
\left.\left\langle\bar{\nu}, s^{i}\right\rangle, S^{i}(\bar{\nu}),\left\langle\bar{\mu}_{i}, \bar{\nu}\right\rangle, S_{\langle\bar{\nu}\rangle}^{i}\right\rangle \\
\left.\left\langle\bar{\mu}_{i-1}, s^{i-1}\right\rangle, S^{i-1}, \ldots,\left\langle\bar{\mu}_{0}, s^{0}\right\rangle, S^{0}\right\rangle .
\end{array}
$$

Note the degenerate case in this definition when $1(\bar{\nu})=0$. In this case $S^{i}(\bar{\nu})=\emptyset$.

Definition 4.4. Let $p, q \in R_{\alpha}$ where

$$
q=\left\langle\left\langle\bar{\mu}_{n}, s^{n}\right\rangle, S^{n}, \ldots,\left\langle\bar{\mu}_{1}, s^{1}\right\rangle, S^{1},\left\langle\bar{\mu}_{0}, s^{0}\right\rangle, S^{0}\right\rangle .
$$

We say that $p$ is a 1-point extension of $q\left(p \leq^{1} q\right)$ if there is $\langle\bar{\nu}\rangle \in S^{i}$ such that $p \leq^{*}(q)_{\langle\bar{\nu}\rangle}$.

Definition 4.5. Let $p, q \in R_{\alpha}$. We say that $p$ is an $n$-point extension of $q\left(p \leq^{n} q\right)$ if there are $p^{n}, \ldots, p^{0}$ such that

$$
p=p^{n} \leq^{1} \ldots \leq^{1} p^{0}=q .
$$

Definition 4.6. Let $p, q \in R_{\alpha}$. We say that $p$ is an extension of $q(p \leq q)$ if there is an $n$ such that $p \leq^{n} q$.

Lemma 4.8 is needed in the proof of Theorem 7.1. Very loosely speaking, Lemma 4.8 means that if "something" happens on a measure-one set for one of the measures, that "something" is happening on a measure-one set for all the measures.

Lemma 4.8 is proved by induction, and Lemma 4.7 is the first case of the induction. 
Lemma 4.7. Suppose $\mathrm{l}\left(\bar{E}_{\alpha}\right)=2, i<2$, and there is a tree $T$ (not an $\bar{E}_{\alpha}$ tree) such that $\operatorname{Lev}_{0}(T) \in E_{\alpha}(i)$, and $\forall\langle\bar{\nu}\rangle \in T T_{\langle\bar{\nu}\rangle}$ is an $\bar{E}_{\alpha}$-tree. Then there is an $\bar{E}_{\alpha}$-tree, $T^{*}$, satisfying

(1) $\forall\langle\bar{\nu}\rangle \in T \cap T^{*} T_{\langle\bar{\nu}\rangle}^{*} \leq T_{\langle\bar{\nu}\rangle}, T^{*}(\bar{\nu}) \leq T(\bar{\nu})$, and

(2) if $p \leq\left\langle\left\langle\bar{E}_{\alpha},\langle\rangle\right\rangle, T^{*}\right\rangle$, then there is $\langle\bar{\mu}\rangle \in T^{*} \cap T$ such that

$$
p \|\left(\left\langle\left\langle\bar{E}_{\alpha},\langle\rangle\right\rangle, T^{*}\right\rangle\right)_{\langle\bar{\mu}\rangle} .
$$

Proof. There are two cases to deal with:

- $A_{0}=\operatorname{Lev}_{0}(T) \in E_{\alpha}(0)$ : If $A_{0} \in E_{\alpha}(1)$, we set $T^{*}=T$, and the proof is finished. So suppose $A_{0} \notin E_{\alpha}(1)$. We would like to build $A_{1} \in E_{\alpha}(1)$. Set

$$
\forall\left\langle\bar{\mu}_{0}\right\rangle \in T A_{\left\langle\bar{\mu}_{0}\right\rangle, 1}=\left\{\left\langle\bar{\mu}_{1}, S\right\rangle \in T_{\left\langle\bar{\mu}_{0}\right\rangle} \mid A_{0} \cap \kappa^{0}\left(\bar{\mu}_{1}\right) \in \bar{\mu}_{1}(0)_{\kappa\left(\bar{\mu}_{1}\right)}\right\} .
$$

Since $A_{0} \in E_{\alpha}(0)$ and $\operatorname{Suc}_{T}\left(\left\langle\bar{\mu}_{0}\right\rangle\right) \in E_{\alpha}(1)$, we get that $A_{\left\langle\bar{\mu}_{0}\right\rangle, 1} \in E_{\alpha}(1)$. Let

$$
A_{1}=\underset{\left\langle\bar{\mu}_{0}\right\rangle \in T}{\triangle^{0}} A_{\left\langle\bar{\mu}_{0}\right\rangle, 1}
$$

We can now construct $T^{*}$ :

$$
\begin{aligned}
& \operatorname{Lev}_{0}\left(T^{*}\right)=A_{0} \cup A_{1}, \\
& \forall \bar{\mu}_{0} \in A_{0} T_{\left\langle\bar{\mu}_{0}\right\rangle}^{*}=T_{\left\langle\bar{\mu}_{0}\right\rangle} \\
& \forall \bar{\mu}_{1} \in A_{1} T_{\left\langle\bar{\mu}_{1}\right\rangle}^{*}=\bigcap_{\left\langle\bar{\mu}_{0}, \bar{\mu}_{1}\right\rangle \in T} T_{\left\langle\bar{\mu}_{0}, \bar{\mu}_{1}\right\rangle} .
\end{aligned}
$$

- $A_{1}=\operatorname{Lev}_{0}(T) \in E_{\alpha}(1):$ If $A_{1} \in E_{\alpha}(0)$, we set $T^{*}=T$ and finish the proof. So assume $A_{1} \notin E_{\alpha}(0)$. We would like to build $A_{0} \in E_{\alpha}(0)$. Set

$$
\begin{aligned}
& S=j(T)(\langle\alpha, E(0)\rangle), \\
& A_{0}=\operatorname{Lev}_{0}(S) \backslash A_{1} .
\end{aligned}
$$

We construct $T^{*}$ :

$$
\begin{aligned}
& \operatorname{Lev}_{0}\left(T^{*}\right)=A_{0} \cup A_{1}, \\
& \forall \bar{\mu}_{1} \in A_{1} T_{\left\langle\bar{\mu}_{1}\right\rangle}^{*}=T_{\left\langle\bar{\mu}_{1}\right\rangle} .
\end{aligned}
$$

We are left with the construction of $T_{\left\langle\bar{\mu}_{0}\right\rangle}^{*}$ for $\bar{\mu}_{0} \in A_{0}$. For all $\bar{\mu} \in A_{0}$, set

$$
\begin{aligned}
& A_{\left\langle\bar{\mu}_{0}\right\rangle, 0}=\operatorname{Suc}_{S}\left(\left\langle\bar{\mu}_{0}\right\rangle\right), \\
& A_{\left\langle\bar{\mu}_{0}\right\rangle, 1}=\left\{\left\langle\bar{\mu}_{1}, T\left(\bar{\mu}_{1}\right)_{\left\langle\bar{\mu}_{0}\right\rangle}\right\rangle \mid\left\langle\bar{\mu}_{1}\right\rangle \in T,\left\langle\bar{\mu}_{0}\right\rangle \in T\left(\bar{\mu}_{1}\right)\right\}, \\
& \operatorname{Suc}_{T^{*}}\left(\left\langle\bar{\mu}_{0}\right\rangle\right)=A_{\left\langle\bar{\mu}_{0}\right\rangle, 0} \cup A_{\left\langle\bar{\mu}_{0}\right\rangle, 1}, \\
& \forall \bar{\mu}_{1} \in A_{\left\langle\bar{\mu}_{0}\right\rangle, 1} T_{\left\langle\bar{\mu}_{0}, \bar{\mu}_{1}\right\rangle}^{*}=T_{\left\langle\bar{\mu}_{1}\right\rangle} .
\end{aligned}
$$

We continue one more level and hope this will convince the reader we indeed can complete $T^{*}$. We are left with the construction of $T_{\left\langle\bar{\mu}_{0}, \bar{\mu}_{1}\right\rangle}^{*}$ for $\left\langle\bar{\mu}_{0}, \bar{\mu}_{1}\right\rangle \in$ $A_{0} \times A_{\left\langle\bar{\mu}_{0}\right\rangle, 0}$. For all $\left\langle\bar{\mu}_{0}, \bar{\mu}_{1}\right\rangle \in A_{0} \times A_{\left\langle\bar{\mu}_{0}\right\rangle, 0}$, set

$$
\begin{aligned}
& A_{\left\langle\bar{\mu}_{0}, \bar{\mu}_{1}\right\rangle, 0}=\operatorname{Suc}_{S}\left(\left\langle\bar{\mu}_{0}, \bar{\mu}_{1}\right\rangle\right), \\
& A_{\left\langle\bar{\mu}_{0}, \bar{\mu}_{1}\right\rangle, 1}=\left\{\left\langle\bar{\mu}_{2}, T\left(\bar{\mu}_{2}\right)_{\left\langle\bar{\mu}_{0}, \bar{\mu}_{1}\right\rangle}\right\rangle \mid\left\langle\bar{\mu}_{2}\right\rangle \in T,\left\langle\bar{\mu}_{0}, \bar{\mu}_{1}\right\rangle \in T\left(\bar{\mu}_{2}\right)\right\}, \\
& \operatorname{Suc}_{T^{*}}\left(\left\langle\bar{\mu}_{0}, \bar{\mu}_{1}\right\rangle\right)=A_{\left\langle\bar{\mu}_{0}, \bar{\mu}_{1}\right\rangle, 0} \cup A_{\left\langle\bar{\mu}_{0}, \bar{\mu}_{1}\right\rangle, 1}, \\
& \forall \bar{\mu}_{2} \in A_{\left\langle\bar{\mu}_{0}, \bar{\mu}_{1}\right\rangle, 1} T_{\left\langle\bar{\mu}_{0}, \bar{\mu}_{1}, \bar{\mu}_{2}\right\rangle}^{*}=T_{\left\langle\bar{\mu}_{2}\right\rangle} .
\end{aligned}
$$


We are left with the construction of $T_{\left\langle\bar{\mu}_{0}, \bar{\mu}_{1}, \bar{\mu}_{2}\right\rangle}$ for $\left\langle\bar{\mu}_{0}, \bar{\mu}_{1}, \bar{\mu}_{2}\right\rangle \in A_{0} \times$ $A_{\left\langle\bar{\mu}_{0}\right\rangle, 0} \times A_{\left\langle\bar{\mu}_{0}, \bar{\mu}_{1}\right\rangle, 0}$, and we hope that by now the continuation is clear.

Lemma 4.8. Let $\xi_{0}<1\left(\bar{E}_{\alpha}\right)$, and let $T$ be a tree such that $\operatorname{Lev}_{0}(T) \in E_{\alpha}\left(\xi_{0}\right)$ and $\forall\langle\bar{\mu}\rangle \in T T_{\langle\bar{\mu}\rangle}$ is an $\bar{E}_{\alpha}$-tree. Then there is an $\bar{E}_{\alpha}$-tree, $T^{*}$, satisfying

(1) $\forall\langle\bar{\nu}\rangle \in T \cap T^{*} T_{\langle\bar{\nu}\rangle}^{*} \leq T_{\langle\bar{\nu}\rangle}, T^{*}(\bar{\nu}) \leq T(\bar{\nu})$, and

(2) if $p \leq\left\langle\left\langle\bar{E}_{\alpha},\langle\rangle\right\rangle, T^{*}\right\rangle$, then there is $\langle\bar{\mu}\rangle \in T^{*} \cap T$ such that

$$
p \|\left(\left\langle\left\langle\bar{E}_{\alpha},\langle\rangle\right\rangle, T^{*}\right\rangle\right)_{\langle\bar{\mu}\rangle} .
$$

Proof. Our induction hypothesis is that this lemma is true for $\bar{\mu}$ 's with $\mathrm{l}(\bar{\mu})<\mathrm{l}\left(\bar{E}_{\alpha}\right)$. The previous lemma is the case for $1(\bar{\mu})=2$.

Let $S=j(T)\left(\bar{E}_{\alpha} \mid \xi_{0}\right)$. The tree $S$ is an $\bar{E}_{\alpha} \mid \xi_{0}$-tree. We extend it step by step to a full $\bar{E}_{\alpha}$-tree as required.

Let

$$
\begin{aligned}
& A_{\xi_{0}}=\operatorname{Lev}_{0}(T), \\
& A_{<\xi_{0}}=\operatorname{Lev}_{0}(S) \backslash A_{\xi_{0}} .
\end{aligned}
$$

For $\xi_{0}<\xi<1\left(\bar{E}_{\alpha}\right)$, do the following:

$$
\begin{aligned}
& N_{\xi}=\operatorname{Ult}\left(V, E_{\alpha}(\xi)\right), \\
& k_{\xi}\left([h]_{E_{\alpha}(\xi)}\right)=j(h)\left(\bar{E}_{\alpha} \mid \xi\right), \\
& N_{\xi}=\operatorname{Ult}\left(V, E_{\left.E_{\alpha}(\xi)\right)} M\right.
\end{aligned}
$$

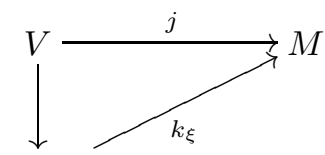

Since

$$
\langle\alpha, E(0), \ldots, E(\tau), \ldots \mid \tau<\xi\rangle=k_{\xi}\left([i d]_{E_{\alpha}(\xi)}\right) \in \operatorname{ran}\left(k_{\xi}\right),
$$

there is in $N_{\xi}$ a preimage for it:

$$
\left\langle\alpha^{\prime}, E^{\prime}(0), \ldots, E^{\prime}\left(\tau^{\prime}\right), \ldots \mid \tau^{\prime}<\xi^{\prime}\right\rangle .
$$

Since $A_{\xi_{0}} \in E_{\alpha}\left(\xi_{0}\right), \xi_{0}<\xi$, we have $\tau^{\prime}<\xi^{\prime}$ such that $A_{\xi_{0}} \in E_{\alpha^{\prime}}^{\prime}\left(\tau^{\prime}\right)$ (where $\left.\alpha^{\prime}=[\kappa(i d)]_{E_{\alpha}(\xi)}\right)$. Taking a function $h_{\xi}$ such that $\left[h_{\xi}\right]_{E_{\alpha}(\xi)}=\tau^{\prime}$, we get

$$
\left\{\bar{\mu} \mid A_{\xi_{0}} \cap \kappa^{0}(\bar{\mu}) \in \bar{\mu}\left(h_{\xi}(\bar{\mu})\right)_{\kappa(\bar{\mu})}\right\} \in E_{\alpha}(\xi) .
$$

For each $\bar{\mu}_{0} \in A_{\xi_{0}}$ we set

$$
\begin{aligned}
& A_{\xi,\left\langle\bar{\mu}_{0}, T\left(\bar{\mu}_{0}\right)\right\rangle}=\left\{\left\langle\bar{\mu}_{1}, R\right\rangle \in T_{\left\langle\bar{\mu}_{0}\right\rangle} \mid A_{\xi_{0}} \cap \kappa^{0}\left(\bar{\mu}_{1}\right) \in \bar{\mu}_{1}\left(h_{\xi}\left(\bar{\mu}_{1}\right)\right)_{\kappa(\bar{\mu})}\right\}, \\
& A_{\xi}^{\prime}=\underset{\left\langle\bar{\mu}_{0}, T\left(\bar{\mu}_{0}\right)\right\rangle}{\triangle^{0}} A_{\xi,\left\langle\bar{\mu}_{0}, T\left(\bar{\mu}_{0}\right)\right\rangle .}
\end{aligned}
$$

For any tree $R$ which appears in a pair $\left\langle\bar{\mu}_{1}, R\right\rangle \in A_{\xi}^{\prime}$ we can invoke our lemma by induction and generate $R^{*}$ which is a $\bar{\mu}_{1}$-tree. Now define $A_{\xi}$ as

$$
\left\langle\bar{\mu}_{1}, R^{*}\right\rangle \in A_{\xi} \Longleftrightarrow\left\langle\bar{\mu}_{1}, R\right\rangle \in A_{\xi}^{\prime} .
$$


When we have $\left\{A_{\xi} \mid \xi_{0}<\xi<\mathrm{l}\left(\bar{E}_{\alpha}\right)\right\}$ we set

$$
\begin{aligned}
& A_{>\xi_{0}}=\bigcup_{\xi_{0}<\xi<1\left(\bar{E}_{\alpha}\right)} A_{\xi} \backslash\left(A_{<\xi_{0}} \cup A_{\xi_{0}}\right), \\
& \operatorname{Lev}_{0}\left(T^{*}\right)=A_{<\xi_{0}} \cup A_{\xi_{0}} \cup A_{>\xi_{0}}, \\
& \forall\left\langle\bar{\mu}_{0}\right\rangle \in A_{\xi_{0}} T_{\left\langle\bar{\mu}_{0}\right\rangle}^{*}=T_{\left\langle\bar{\mu}_{0}\right\rangle}, \\
& \forall\left\langle\bar{\mu}_{1}\right\rangle \in A_{>\xi_{0}} T_{\left\langle\bar{\mu}_{1}\right\rangle}^{*}=\bigcap T_{\left\langle\bar{\mu}_{0}, \bar{\mu}_{1}\right\rangle} .
\end{aligned}
$$

We are left to define $T_{\left\langle\bar{\mu}_{0}\right\rangle}$ for $\left\langle\bar{\mu}_{0}\right\rangle \in A_{<\xi_{0}}$. For each $\bar{\mu}_{0} \in A_{<\xi_{0}}$, set

$$
\begin{aligned}
& A_{\left\langle\bar{\mu}_{0}\right\rangle, \xi_{0}}=\left\{\left\langle\bar{\mu}_{1}, R_{\left\langle\bar{\mu}_{0}\right\rangle}\right\rangle \mid\left\langle\bar{\mu}_{1}, R\right\rangle \in A_{\xi_{0}},\left\langle\bar{\mu}_{0}\right\rangle \in R\right\}, \\
& A_{\left\langle\bar{\mu}_{0}\right\rangle,<\xi_{0}}=\operatorname{Suc}_{S}\left(\left\langle\bar{\mu}_{0}\right\rangle\right) \backslash A_{\left\langle\bar{\mu}_{0}\right\rangle, \xi_{0}} .
\end{aligned}
$$

For each $\bar{\mu}_{1} \in A_{\xi_{0}}$ we set

$$
\begin{aligned}
& A_{\left\langle\bar{\mu}_{0}\right\rangle, \xi,\left\langle\bar{\mu}_{1}, T\left(\bar{\mu}_{1}\right)_{\left\langle\bar{\mu}_{0}\right\rangle}\right\rangle}=\left\{\left\langle\bar{\mu}_{2}, R\right\rangle \in T_{\left\langle\bar{\mu}_{1}\right\rangle} \mid A_{\xi_{0}} \cap \kappa^{0}\left(\bar{\mu}_{2}\right) \in \bar{\mu}_{2}\left(h_{\xi}\left(\bar{\mu}_{2}\right)\right)_{\kappa\left(\bar{\mu}_{2}\right)}\right\}, \\
& A_{\left\langle\bar{\mu}_{0}\right\rangle, \xi}^{\prime}=\underset{\left\langle\bar{\mu}_{1}, T\left(\bar{\mu}_{1}\right)_{\left\langle\bar{\mu}_{0}\right\rangle}\right\rangle}{\triangle_{\left\langle\mu_{0}\right\rangle, \xi,\left\langle\bar{\mu}_{1}, T\left(\bar{\mu}_{1}\right)_{\left\langle\bar{\mu}_{0}\right\rangle}\right\rangle}} A_{\langle\bar{\mu}}
\end{aligned}
$$

We define

$$
\left\langle\bar{\mu}_{0}, R^{*}\right\rangle \in A_{\left\langle\bar{\mu}_{0}\right\rangle, \xi} \Longleftrightarrow\left\langle\bar{\mu}_{0}, R\right\rangle \in A_{\left\langle\bar{\mu}_{0}\right\rangle, \xi}^{\prime}
$$

where $R^{*}$ is generated from $R$ using the current lemma by induction. Now we set

$$
\begin{aligned}
& A_{\left\langle\bar{\mu}_{0}\right\rangle,>\xi_{0}}=\bigcup_{\xi_{0}<\xi<1\left(\bar{E}_{\alpha}\right)} A_{\left\langle\bar{\mu}_{0}\right\rangle, \xi} \backslash\left(A_{\left\langle\bar{\mu}_{0}\right\rangle,<\xi_{0}} \cup A_{\left\langle\bar{\mu}_{0}\right\rangle, \xi_{0}}\right), \\
& \operatorname{Suc}_{T^{*}}\left(\left\langle\bar{\mu}_{0}\right\rangle\right)=A_{\left\langle\bar{\mu}_{0}\right\rangle,<\xi_{0}} \cup A_{\left\langle\bar{\mu}_{0}\right\rangle, \xi_{0}} \cup A_{\left\langle\bar{\mu}_{0}\right\rangle,>\xi_{0}}, \\
& \forall\left\langle\bar{\mu}_{1}\right\rangle \in A_{\bar{\mu}_{0}, \xi_{0}} T_{\left\langle\bar{\mu}_{0}, \bar{\mu}_{1}\right\rangle}^{*}=T_{\left\langle\bar{\mu}_{1}\right\rangle}, \\
& \forall\left\langle\bar{\mu}_{2}\right\rangle \in A_{\bar{\mu}_{0},>\xi_{0}} T_{\left\langle\bar{\mu}_{0}, \bar{\mu}_{2}\right\rangle}^{*}=\bigcap T_{\left\langle\bar{\mu}_{1}, \bar{\mu}_{2}\right\rangle} .
\end{aligned}
$$

This leaves us with the definition of $T_{\left\langle\bar{\mu}_{0}, \bar{\mu}_{1}\right\rangle}$ for $\left\langle\bar{\mu}_{0}, \bar{\mu}_{1}\right\rangle \in A_{<\xi_{0}} \times A_{\left\langle\bar{\mu}_{0}\right\rangle,<\xi_{0}}$, which is done exactly as in this step.

Lemma 4.10 is needed in the proof of Theorem 6.1. Loosely speaking, it says that if "something" happens on all extensions which are taken from $\operatorname{dom} T$, then that "something" happens on all extensions from $T$.

Lemma 4.10 is proved by induction where Lemma 4.9 is the first case.

Lemma 4.9. Assume $1\left(\bar{E}_{\alpha}\right)=2$, and let $T$ be an $\bar{E}_{\alpha}$-tree. Then there is $T^{*} \leq T$ such that, if

$$
p \leq\left\langle\left\langle\bar{E}_{\alpha},\langle\rangle\right\rangle, T^{*}\right\rangle
$$

then there is $\left\langle\bar{\nu}_{1}, \ldots, \bar{\nu}_{n}\right\rangle \in T$ such that

$$
p \leq^{*}\left(\left\langle\left\langle\bar{E}_{\alpha},\langle\rangle\right\rangle, T\right\rangle\right)_{\left\langle\bar{\nu}_{1}, \ldots, \bar{\nu}_{n}\right\rangle} \cdot
$$

Proof. As is usual in this section, the proof is done level by level. Let us set $T^{1}=T$. It is trivially true that if

$$
p \leq^{1}\left\langle\left\langle\bar{E}_{\alpha},\langle\rangle\right\rangle, T^{1}\right\rangle,
$$

then there is $\left\langle\bar{\nu}_{1}\right\rangle \in T^{1}=T$ such that

$$
p \leq^{*}\left(\left\langle\left\langle\bar{E}_{\alpha},\langle\rangle\right\rangle, T^{1}\right\rangle\right)_{\left\langle\bar{\nu}_{1}\right\rangle} .
$$


We continue to the second level. Let us set

$$
\begin{aligned}
& A_{\left\langle\bar{\nu}_{1}, S^{1}\right\rangle}=\operatorname{Suc}_{T^{1}}\left(\left\langle\bar{\nu}_{1}\right\rangle\right), \\
& A_{\langle\rangle}=\underset{\left\langle\bar{\nu}_{1}, S^{1}\right\rangle}{\triangle^{0}} A_{\left\langle\bar{\nu}_{1}, S^{1}\right\rangle}, \\
& B_{\langle\rangle}=\left\{\left\langle\bar{\nu}_{2}\right\rangle \mid l\left(\bar{\nu}_{2}\right)=0 \text { or } \operatorname{Lev}_{0}\left(T^{1}\right) \cap \kappa^{0}\left(\bar{\nu}_{2}\right) \in \bar{\nu}_{2}\right\}, \\
& \operatorname{Lev}_{0}\left(T^{(0)}\right)=A_{\langle\rangle} \cap B_{\langle\rangle}, \\
& T_{\left\langle\bar{\nu}_{2}\right\rangle}^{(0)}=\bigcap_{\left\langle\bar{\nu}_{1}\right\rangle \in T^{(0)}\left(\bar{\nu}_{2}\right)} T_{\left\langle\bar{\nu}_{1}, \bar{\nu}_{2}\right\rangle}^{1}, \\
& T^{2}=T^{1} \cap T^{(0)} .
\end{aligned}
$$

Let us assume that

$$
p \leq^{2}\left\langle\left\langle\bar{E}_{\alpha},\langle\rangle\right\rangle, T^{2}\right\rangle .
$$

There are two cases to consider here:

(1) $p \leq^{2}\left(\left\langle\left\langle\bar{E}_{\alpha},\langle\rangle\right\rangle, T^{2}\right\rangle\right)_{\left\langle\bar{\nu}_{1}, \bar{\nu}_{2}\right\rangle}$ where $\left\langle\bar{\nu}_{1}, \bar{\nu}_{2}\right\rangle \in T^{2}$ : At once we have $\left\langle\bar{\nu}_{1}, \bar{\nu}_{2}\right\rangle \in$ $T^{1} \leq T$.

(2) $p \leq^{2}\left(\left\langle\left\langle\bar{E}_{\alpha},\langle\rangle\right\rangle, T^{2}\right\rangle\right)_{\left\langle\bar{\nu}_{2}, \bar{\nu}_{1}\right\rangle}$ where $\left\langle\bar{\nu}_{2}\right\rangle \in T^{2},\left\langle\bar{\nu}_{1}\right\rangle \in T^{2}\left(\bar{\nu}_{2}\right)$ : By construction, $\forall\langle\bar{\mu}\rangle \in T^{2}\left(\bar{\nu}_{2}\right)\left\langle\bar{\nu}_{2}\right\rangle \in T_{\langle\bar{\mu}\rangle}^{1}$. Since $\left\langle\bar{\nu}_{1}\right\rangle \in T^{2}\left(\bar{\nu}_{2}\right)$, we get $\left\langle\bar{\nu}_{1}, \bar{\nu}_{2}\right\rangle \in$ $T^{1} \leq T$.

We show how to continue to the third level. Let us set

$$
\begin{aligned}
& A_{\left\langle\bar{\nu}_{1}, S^{1}, \bar{\nu}_{2}, S^{2}\right\rangle}=\operatorname{Suc}_{T^{2}}\left(\left\langle\bar{\nu}_{1}, \bar{\nu}_{2}\right\rangle\right), \\
& A_{\left\langle\bar{\nu}_{1}, S^{1}\right\rangle}=\underset{\left\langle\bar{\nu}_{2}, S^{2}\right\rangle}{\triangle^{0}} A_{\left\langle\bar{\nu}_{1}, S^{1}, \bar{\nu}_{2}, S^{2}\right\rangle}, \\
& B_{\left\langle\bar{\nu}_{1}, S^{1}\right\rangle}=\left\{\left\langle\bar{\nu}_{3}\right\rangle \mid \mathrm{l}\left(\bar{\nu}_{3}\right)=0 \text { or } \operatorname{Suc}_{T^{2}}\left(\left\langle\bar{\nu}_{1}, S^{1}\right\rangle\right) \cap \kappa^{0}\left(\bar{\nu}_{3}\right) \in \bar{\nu}_{3}\right\}, \\
& \operatorname{Lev}_{0}\left(T^{(0)}\right)=\operatorname{Lev}_{0}\left(T^{2}\right) \text {, } \\
& \operatorname{Suc}_{T^{(0)}}\left(\left\langle\bar{\nu}_{1}\right\rangle\right)=A_{\left\langle\bar{\nu}_{1}, S^{1}\right\rangle} \cap B_{\left\langle\bar{\nu}_{1}, S^{1}\right\rangle}, \\
& T_{\left\langle\bar{\nu}_{1}, \bar{\nu}_{3}\right\rangle}^{(0)}=\bigcap_{\left\langle\bar{\nu}_{2}\right\rangle \in T_{\left\langle\bar{\nu}_{1}\right\rangle}^{(0)}\left(\bar{\nu}_{3}\right)} T_{\left\langle\bar{\nu}_{1}, \bar{\nu}_{2}, \bar{\nu}_{3}\right\rangle}^{2}, \\
& A_{\langle\rangle}=\underset{\left\langle\bar{\nu}_{1}, S^{1}\right\rangle}{\triangle^{0}} A_{\left\langle\bar{\nu}_{1}, S^{1}\right\rangle}, \\
& B_{\langle\rangle}=\left\{\left\langle\bar{\nu}_{3}\right\rangle \mid \mathrm{l}\left(\bar{\nu}_{3}\right)=0 \text { or } \operatorname{Lev}_{0}\left(T^{2}\right) \cap \kappa^{0}\left(\bar{\nu}_{3}\right) \in \bar{\nu}_{3}\right\}, \\
& \operatorname{Lev}_{0}\left(T^{(1)}\right)=A_{\langle\rangle} \cap B_{\langle\rangle}, \\
& \operatorname{Suc}_{T^{(1)}}\left(\left\langle\bar{\nu}_{3}\right\rangle\right)=\bigcap_{\left\langle\bar{\nu}_{1}, \bar{\nu}_{2}\right\rangle \in T^{(0)}\left(\bar{\nu}_{3}\right)} T_{\left\langle\bar{\nu}_{1}, \bar{\nu}_{2}, \bar{\nu}_{3}\right\rangle}^{2}, \\
& T^{3}=T^{2} \cap T^{(0)} \cap T^{(1)} .
\end{aligned}
$$

Let us assume that

$$
p \leq^{3}\left\langle\left\langle\bar{E}_{\alpha},\langle\rangle\right\rangle, T^{3}\right\rangle
$$

There are three cases to consider here:

(1) $p \leq^{*}\left(\left\langle\left\langle\bar{E}_{\alpha},\langle\rangle\right\rangle, T^{3}\right\rangle\right)_{\left\langle\bar{\nu}_{1}, \bar{\nu}_{2}, \bar{\nu}_{3}\right\rangle}$ where $\left\langle\bar{\nu}_{1}, \bar{\nu}_{2}, \bar{\nu}_{3}\right\rangle \in T^{3}$ : At once we get $\left\langle\bar{\nu}_{1}, \bar{\nu}_{2}, \bar{\nu}_{3}\right\rangle \in T$. 
(2) $p \leq^{*}\left(\left\langle\left\langle\bar{E}_{\alpha},\langle\rangle\right\rangle, T^{3}\right\rangle\right)_{\left\langle\bar{\nu}_{1}, \bar{\nu}_{3}, \bar{\nu}_{2}\right\rangle}$ where $\left\langle\bar{\nu}_{1}, \bar{\nu}_{3}\right\rangle \in T^{3},\left\langle\bar{\nu}_{2}\right\rangle \in T_{\left\langle\bar{\nu}_{1}\right\rangle}^{3}\left(\bar{\nu}_{3}\right):$ In this case $\forall\langle\bar{\mu}\rangle \in T_{\left\langle\bar{\nu}_{1}\right\rangle}^{3}\left(\bar{\nu}_{3}\right)\left\langle\bar{\nu}_{3}\right\rangle \in T_{\left\langle\bar{\nu}_{1}, \bar{\mu}\right\rangle}^{3}$. Since $\left\langle\bar{\nu}_{2}\right\rangle \in T_{\left\langle\bar{\nu}_{1}\right\rangle}^{3}\left(\bar{\nu}_{3}\right)$, we get $\left\langle\bar{\nu}_{3}\right\rangle \in T_{\left\langle\bar{\nu}_{1}, \bar{\nu}_{2}\right\rangle} \cdot$

(3) $p \leq^{*}\left(\left\langle\left\langle\bar{E}_{\alpha},\langle\rangle\right\rangle, T^{3}\right\rangle\right)_{\left\langle\bar{\nu}_{3}, \bar{\nu}_{1}, \bar{\nu}_{2}\right\rangle}$ where $\left\langle\bar{\nu}_{3}\right\rangle \in T^{3},\left\langle\bar{\nu}_{1}, \bar{\nu}_{2}\right\rangle \in T^{3}\left(\bar{\nu}_{3}\right)$ : Then

$$
\forall\left\langle\bar{\mu}_{1}, \bar{\mu}_{2}\right\rangle \in T^{3}\left(\bar{\nu}_{3}\right)\left\langle\bar{\nu}_{3}\right\rangle \in T_{\left\langle\bar{\mu}_{1}, \bar{\mu}_{2}\right\rangle}^{3} ; \text { hence }\left\langle\bar{\nu}_{3}\right\rangle \in T_{\left\langle\bar{\nu}_{1}, \bar{\nu}_{2}\right\rangle} \text {. }
$$

In this way we continue to all levels.

Lemma 4.10. Let $T$ be an $\bar{E}_{\alpha}$ tree. Then there is $T^{*} \leq T$ such that if

$$
p \leq\left\langle\left\langle\bar{E}_{\alpha},\langle\rangle\right\rangle, T^{*}\right\rangle
$$

then there is $\left\langle\bar{\nu}_{1}, \ldots, \bar{\nu}_{n}\right\rangle \in T$ such that

$$
p \leq^{*}\left(\left\langle\left\langle\bar{E}_{\alpha},\langle\rangle\right\rangle, T\right\rangle\right)_{\left\langle\bar{\nu}_{1}, \ldots, \bar{\nu}_{n}\right\rangle} .
$$

Proof. The proof is by induction on $\mathrm{l}\left(\bar{E}_{\alpha}\right)$. The first case was done in Lemma 4.9 The proof is almost the same. We just make sure to invoke the induction hypothesis while repeating the construction.

Construction of $T^{1}$ and $T^{2}$ is exactly as in Lemma 4.9. We show the construction at the 3rd level.

Let us set

$$
\begin{aligned}
& A_{\left\langle\bar{\nu}_{1}, S^{1}, \bar{\nu}_{2}, S^{2}\right\rangle}=\operatorname{Suc}_{T^{2}}\left(\left\langle\bar{\nu}_{1}, \bar{\nu}_{2}\right\rangle\right), \\
& A_{\left\langle\bar{\nu}_{1}, S^{1}\right\rangle}=\underset{\left\langle\bar{\nu}_{2}, S^{2}\right\rangle}{\Delta} A_{\left\langle\bar{\nu}_{1}, S^{1}, \bar{\nu}_{2}, S^{2}\right\rangle}, \\
& B_{\left\langle\bar{\nu}_{1}, S^{1}\right\rangle}=\left\{\left\langle\left\langle\bar{\nu}_{3}\right\rangle\right| \mathrm{l}\left(\bar{\nu}_{3}\right)=0 \text { or } \operatorname{Suc}_{T^{2}}\left(\left\langle\bar{\nu}_{1}, S^{1}\right\rangle\right) \cap \kappa^{0}\left(\bar{\nu}_{3}\right) \in \bar{\nu}_{3}\right\}, \\
& \operatorname{Lev}_{0}\left(T^{(0)}\right)=\operatorname{Lev}_{0}\left(T^{2}\right), \\
& \operatorname{Suc}_{T^{(0)}}\left(\left\langle\bar{\nu}_{1}\right\rangle\right)=A_{\left\langle\bar{\nu}_{1}, S^{1}\right\rangle} \cap B_{\left\langle\bar{\nu}_{1}, S^{1}\right\rangle}, \\
& T_{\left\langle\bar{\nu}_{1}, \bar{\nu}_{3}\right\rangle}^{(0)}=\bigcap_{\left\langle\bar{\nu}_{2}\right\rangle \in T_{\left\langle\bar{\nu}_{1}\right\rangle}^{(0)}\left(\bar{\nu}_{3}\right)} T_{\left\langle\bar{\nu}_{1}, \bar{\nu}_{2}, \bar{\nu}_{3}\right\rangle}^{2}, \\
& A_{\langle\rangle}^{\prime}=\triangle_{\left\langle\bar{\nu}_{1}, S^{1}\right\rangle}^{0} A_{\left\langle\bar{\nu}_{1}, S^{1}\right\rangle}, \\
& A_{\langle\rangle}=\left\{\left\langle\bar{\nu}_{3}, S^{3}\right\rangle \mid\left\langle\bar{\nu}_{3}, S^{3 \prime}\right\rangle \in A_{\langle\rangle}^{\prime} \text { and } S \text { is generated from } S^{3 \prime} \text { by induction }\right\}, \\
& B_{\langle\rangle}=\left\{\left\langle\bar{\nu}_{3}\right\rangle \mid l\left(\bar{\nu}_{3}\right)=0 \text { or Lev } \operatorname{Lev}_{0}\left(T^{2}\right) \cap \kappa^{0}\left(\bar{\nu}_{3}\right) \in \bar{\nu}_{3}\right\}, \\
& \operatorname{Lev}_{0}\left(T^{(1)}\right)=A_{\langle\rangle} \cap B_{\langle\rangle}, \\
& \operatorname{Suc}_{T^{(1)}}\left(\left\langle\bar{\nu}_{3}\right\rangle\right)=\bigcap_{\left\langle\bar{\nu}_{1}, \bar{\nu}_{2}\right\rangle \in T^{(0)}\left(\bar{\nu}_{3}\right)} T_{\left\langle\bar{\nu}_{1}, \bar{\nu}_{2}, \bar{\nu}_{3}\right\rangle}^{2}, \\
& T^{3}=T^{2} \cap T^{(0)} \cap T^{(1)} .
\end{aligned}
$$

Let us assume that

$$
p_{2} \frown p_{1} \frown p_{0}=p \leq^{3}\left\langle\left\langle\bar{E}_{\alpha},\langle\rangle\right\rangle, T^{3}\right\rangle .
$$

There are three cases to consider here:

(1) $p \leq^{*}\left(\left\langle\left\langle\bar{E}_{\alpha},\langle\rangle\right\rangle, T^{3}\right\rangle\right)_{\left\langle\bar{\nu}_{1}, \bar{\nu}_{2}, \bar{\nu}_{3}\right\rangle}$ where $\left\langle\bar{\nu}_{1}, \bar{\nu}_{2}, \bar{\nu}_{3}\right\rangle \in T^{3}$ : At once we get $\left\langle\bar{\nu}_{1}, \bar{\nu}_{2}, \bar{\nu}_{3}\right\rangle \in T$. 
(2) $p \leq^{*}\left(\left\langle\left\langle\bar{E}_{\alpha},\langle\rangle\right\rangle, T^{3}\right\rangle\right)_{\left\langle\bar{\nu}_{1}, \bar{\nu}_{3}, \bar{\nu}_{2}\right\rangle}$ where $\left\langle\bar{\nu}_{1}, \bar{\nu}_{3}\right\rangle \in T^{3},\left\langle\bar{\nu}_{2}\right\rangle \in T_{\left\langle\bar{\nu}_{1}\right\rangle}^{3}\left(\bar{\nu}_{3}\right):$ In this case, $\forall\langle\bar{\mu}\rangle \in T_{\left\langle\bar{\nu}_{1}\right\rangle}^{3}\left(\bar{\nu}_{3}\right)\left\langle\bar{\nu}_{3}\right\rangle \in T_{\left\langle\bar{\nu}_{1}, \bar{\mu}\right\rangle}^{3}$. Since $\left\langle\bar{\nu}_{2}\right\rangle \in T_{\left\langle\bar{\nu}_{1}\right\rangle}^{3}\left(\bar{\nu}_{3}\right)$, we get $\left\langle\bar{\nu}_{3}\right\rangle \in T_{\left\langle\bar{\nu}_{1}, \bar{\nu}_{2}\right\rangle}$.

(3) $p \leq^{2}\left(\left\langle\left\langle\bar{E}_{\alpha},\langle\rangle\right\rangle, T^{3}\right\rangle\right)_{\left\langle\bar{\nu}_{3}\right\rangle}$ where $\left\langle\bar{\nu}_{3}\right\rangle \in T^{3}$ and $p_{2} \frown p_{1} \leq^{2}\left\langle\left\langle\bar{\nu}_{3},\langle\rangle\right\rangle, T^{3}\left(\bar{\nu}_{3}\right)\right\rangle$. By induction there is $\left\langle\bar{\nu}_{1}, \bar{\nu}_{2}\right\rangle \in T\left(\bar{\nu}_{3}\right)$ such that

$$
p_{2} \frown p_{1} \leq^{*}\left(\left\langle\left\langle\bar{\nu}_{3},\langle\rangle\right\rangle, T^{2}\left(\bar{\nu}_{3}\right)\right\rangle\right)_{\left\langle\bar{\nu}_{1}, \bar{\nu}_{2}\right\rangle} .
$$

By construction, $\forall\left\langle\bar{\mu}_{1}, \bar{\mu}_{2}\right\rangle \in T^{2}\left(\bar{\nu}_{3}\right)\left\langle\bar{\nu}_{3}\right\rangle \in T_{\left\langle\bar{\mu}_{1}, \bar{\mu}_{2}\right\rangle}^{2}$; hence $\left\langle\bar{\nu}_{3}\right\rangle \in T_{\left\langle\bar{\nu}_{1}, \bar{\nu}_{2}\right\rangle}$.

In this way we continue to all levels.

\section{Basic Properties of $P_{\bar{E}}$}

Claim 5.1. $P_{\bar{E}}$ satisfies $\kappa^{++}$-c.c.

Proof. The usual $\Delta$-lemma argument on the support will do.

Claim 5.2. Let $p \in P_{\bar{E}}, P^{*}=\left\{q \leq_{R} p \mid p \in P_{\bar{E}}\right\}$. Then

(1) $\left\langle P^{*}, \leq_{R}\right\rangle$ satisfies $\kappa^{+}$-c.c., and

(2) $\left\langle P^{*}, \leq_{R}\right\rangle$ is sub-forcing of $\left\langle P_{\bar{E}} / p, \leq\right\rangle$.

Proof. Showing $\kappa^{+}$-c.c. is trivial.

Showing that $P^{*}$ is sub-forcing of $P_{\bar{E}} / p$ amounts to showing that any maximal anti-chain of $P^{*}$ is also a maximal anti-chain of $P_{\bar{E}} / p$.

Let $A$ be a maximal anti-chain of $P^{*}$. Let $q \in P_{\bar{E}} / p$. Then $q \leq p$, and there is $r^{\prime} \in P^{*}$ such that $q \leq^{*} r^{\prime} \leq_{R} p$. Assume that $r^{\prime}=r_{n}^{\prime} \frown \ldots \frown r_{0}^{\prime}$. Then also $q=q_{n} \frown \ldots \frown q_{0}$. Let $r_{i}$ be $r_{i}^{\prime}$ with $T_{i}^{r_{i}^{\prime}}$ replaced by $T^{r_{i}^{\prime}} \cap \pi_{\mathrm{mc}\left(q_{i}\right), \operatorname{mc}\left(r_{i}^{\prime}\right)}\left(T^{q_{i}}\right)$ and $r=r_{n} \frown \ldots \frown r_{0}$. Since $r \in P^{*}$ and $A$ is a maximal anti-chain, there is an $a \in A$ such that $a \| r$. Take $s \leq_{R} a, r$. Considering how we constructed $r$ from $r^{\prime}$, we must have $t \leq \leq^{*} s$ such that $t \leq q$. Hence $q \| a$. So we get that $A$ is a maximal anti-chain of $P_{\bar{E}} / p$.

Claim 5.3. Let $p \in P_{\bar{E}}, P^{*}=\left\{q \leq_{R} p \mid p \in P_{\bar{E}}\right\}$. Then there is $r \in R_{\mathrm{mc}(p)}$ such that $P^{*} \simeq R_{\mathrm{mc}(p)} / r$.

Proof. For simplicity, assume that $p=p_{0}$. Then we set $r=\left\langle\left\langle\operatorname{mc}\left(p_{0}\right), p_{0}^{\mathrm{mc}}\right\rangle, T^{p}\right\rangle$.

We give the isomorphism: The image of $q \in P^{*}$ is $s \in\left\langle R_{\mathrm{mc}(p)} / r, \leq\right\rangle$ such that

(1) $q \leq^{*} s$, and

(2) $T^{s_{i}}=T^{q_{i}}$ where $s=s_{n} \frown \ldots \frown s_{0}, q=q_{n} \frown \ldots \frown q_{0}$.

Let $G$ be $P_{\bar{E}^{-}}$generic.

Definition 5.4. $\bar{E}_{G}$ is the enumeration of $\left\{\bar{E}\left(p_{k}\right) \mid p_{n} \frown \ldots \frown p_{0} \in G\right\}$ ordered increasingly by $\kappa^{0}\left(\bar{E}\left(p_{k}\right)\right)$.

Definition 5.5. Let $\zeta<\operatorname{otp}\left(\bar{E}_{G}\right)$. Then

(1) $G \mid \zeta=\left\{p_{n} \frown \ldots \frown p_{k} \mid p_{n} \frown \ldots \frown p_{k} \frown \ldots \frown p_{0} \in G, \bar{E}\left(p_{k}\right)=\bar{E}_{G}(\zeta)\right\}$,

(2) $G \backslash \zeta=\left\{p_{k-1} \frown \ldots \frown p_{0} \mid p_{n} \frown \ldots \frown p_{k} \frown \ldots \frown p_{0} \in G, \bar{E}\left(p_{k}\right)=\bar{E}_{G}(\zeta)\right\}$.

\section{Definition 5.6.}

$$
\begin{aligned}
& M_{G}^{\alpha}=\bigcup\left\{p^{\bar{E}_{\alpha}} \mid p \in G, \bar{E}_{\alpha} \in \operatorname{supp} p\right\}, \\
& C_{G}^{\alpha}=\left\{\kappa(\bar{\mu}) \mid \bar{\mu} \in M_{G}^{\alpha}\right\} .
\end{aligned}
$$


Proposition 5.7. (1) $C_{G}^{\kappa}$ is a club in $\kappa$.

(2) $C_{G}^{\alpha}$ is unbounded in $\kappa$.

(3) $\alpha \neq \beta \Longrightarrow C_{G}^{\alpha} \neq C_{G}^{\beta}$.

Proof. The first two claims are immediate, since these are sequences generated by Radin forcing.

The last is by density and noticing that for $p \in P_{\bar{E}}$ when $\bar{\nu} \in T^{p}$ is permitted for $p^{\bar{E}_{\alpha}}, p^{\bar{E}_{\beta}}$ we required $\pi_{\mathrm{mc}(p), \bar{E}_{\alpha}}(\bar{\nu}) \neq \pi_{\mathrm{mc}(p), \bar{E}_{\beta}}(\bar{\nu})$.

\section{Homogeneity in Dense Open Subsets}

Our aim in this section is to prove the following

Theorem 6.1. Let $D \subseteq P_{\bar{E}}$ be dense open and $p=p_{k} \frown \ldots \frown p_{0} \in P_{\bar{E}}$. Then there is $p^{*} \leq^{*} p$ such that

$$
\begin{aligned}
\exists S^{k} \exists n_{k} \forall\left\langle\bar{\nu}_{k, 1}, \ldots, \bar{\nu}_{k, n_{k}}\right\rangle \in S^{k} \ldots & \exists S^{0} \exists n_{0} \forall\left\langle\bar{\nu}_{0,1}, \ldots, \bar{\nu}_{0, n_{0}}\right\rangle \in S^{0} \\
& \left(p_{k}^{*}\right)_{\left\langle\bar{\nu}_{k, 1}, \ldots, \bar{\nu}_{k, n_{k}}\right\rangle} \frown \ldots \frown\left(p_{0}^{*}\right)_{\left\langle\bar{\nu}_{0,1}, \ldots, \bar{\nu}_{0, n_{0}}\right\rangle} \in D
\end{aligned}
$$

where

(1) $S^{i} \subseteq T^{p_{i}^{*}} \uparrow\left[V_{\kappa}\right]^{n_{i}}$, and

(2) $\forall l<n_{i} \forall\left\langle\bar{\nu}_{1}, \ldots, \bar{\nu}_{l}\right\rangle \in S^{i} \exists \xi \operatorname{Suc}_{S^{i}}\left(\left\langle\bar{\nu}_{1}, \ldots, \bar{\nu}_{l}\right\rangle\right) \in E_{\mathrm{mc}\left(p_{i}^{*}\right)}(\xi)$.

The proof is done by a series of lemmas.

Definition 6.2. Let $p \in P_{\bar{E}}^{*}$. Let $s$ be a function such that $\operatorname{dom} s \subseteq \bar{E}$ and for all $\bar{\alpha}, \bar{\beta} \in \operatorname{dom} s, \bar{\alpha} \neq \bar{\beta}$,

(1) $s(\bar{\alpha})$ is an extender sequence,

(2) $\mathrm{l}(s(\bar{\alpha}))=\mathrm{l}(s(\bar{\beta}))$,

(3) $\kappa^{0}(s(\bar{\alpha}))=\kappa^{0}(s(\bar{\beta}))$,

(4) $s(\bar{\alpha}) \neq s(\bar{\beta})$.

We define $(p)_{\langle s\rangle}$ to be $p_{1}^{\prime} \frown p_{0}^{\prime}$ where

(1) $\operatorname{supp} p_{0}^{\prime}=\operatorname{supp} p$,

(2) $\forall \bar{\alpha} \in \operatorname{supp} p_{0}^{\prime}$

$$
p_{0}^{\prime \bar{\alpha}}= \begin{cases}s(\bar{\alpha}), & \bar{\alpha} \in \operatorname{dom} s, \max \kappa\left(p^{\bar{\alpha}}\right)<\kappa^{0}(s(\bar{\alpha})), \mathrm{l}(s(\bar{\alpha}))>0, \\ p_{0}^{\bar{\alpha}} s(\bar{\alpha}), & \bar{\alpha} \in \operatorname{dom} s, \max \kappa\left(p^{\bar{\alpha}}\right)<\kappa^{0}(s(\bar{\alpha})), \mathrm{l}(s(\bar{\alpha}))=0, \\ p^{\bar{\alpha}}, & \text { otherwise, }\end{cases}
$$

(3) if $s(\operatorname{mc}(p)) \in T^{p}$, then $T^{p_{0}^{\prime}}=T_{\langle s(\operatorname{mc}(p))\rangle}^{p}$; otherwise we leave $T^{p_{0}^{\prime}}$ undefined,

(4) if $\forall \bar{\alpha} \in \operatorname{dom} s \mathrm{l}(s(\bar{\alpha}))=0$, then $p_{1}^{\prime}=\emptyset$,

(5) if $\forall \bar{\alpha} \in \operatorname{dom} s \mathrm{l}(s(\bar{\alpha}))>0$, then

(5.1) $\operatorname{supp} p_{1}^{\prime}=\left\{s(\bar{\alpha}) \mid \bar{\alpha} \in \operatorname{supp} p \cap \operatorname{dom} s, \max \kappa\left(p^{\bar{\alpha}}\right)<\kappa^{0}(s(\bar{\alpha}))\right\}$,

(5.2) $\forall \bar{\alpha} \in \operatorname{supp} p_{0}^{\prime} \forall s(\bar{\alpha}) \in \operatorname{supp} p_{1}^{\prime} p_{1}^{\prime s(\bar{\alpha})}=p^{\bar{\alpha}}$,

(5.3) if $s(\operatorname{mc}(p)) \in T^{p}$, then $T^{p_{1}^{\prime}}=T^{p}(s(\operatorname{mc}(p)))$; otherwise we leave $T^{p_{1}^{\prime}}$ undefined.

Definition 6.3. Let $p \in P_{\bar{E}}^{*}$. Let $s$ be a function with $\operatorname{dom} s=1, \ldots, n$ such that for all $i, s(i)$ satisfies Definition 6.2. Then we define $(p)_{\langle s\rangle}$ as $p^{n}$ where $p^{n}$ is defined 
by induction as follows:

$$
\begin{aligned}
& p^{0}=p,
\end{aligned}
$$

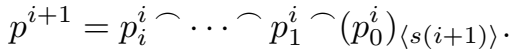

We note the following: If $\left\langle\bar{\nu}_{1}, \ldots, \bar{\nu}_{n}\right\rangle \in T^{p}$ and for all $1 \leq i \leq n$ we set

$$
s(i)=\left\{\left\langle\bar{\alpha}, \pi_{\mathrm{mc}(p), \bar{\alpha}}\left(\bar{\nu}_{i}\right)\right\rangle \mid \bar{\alpha} \in \operatorname{supp} p\right\},
$$

then

$$
(p)_{\left\langle\bar{\nu}_{1}, \ldots, \bar{\nu}_{n}\right\rangle}=(p)_{\langle s\rangle} .
$$

We use this operation also in cases where $p$ is not strictly a condition. That is, if $p \cup\{T\} \in P_{\bar{E}}$, we also use $(p)_{\langle s\rangle}$. In this case we ignore the trees in the definition.

This definition is used in the proof of the homogeneity, because we do not know beforehand what a legitimate extension is. By checking all possible $\mu$ 's we check all legitimate conditions which might be extensions.

Claim 6.4. Let $D$ be dense open in $P_{\bar{E}} / P_{\bar{\epsilon}}, p=p_{0} \in P_{\bar{E}} / P_{\bar{\epsilon}}, 0<n<\omega$. Then there is $p^{*} \leq^{*} p$ such that one and only one of the following is true:

(1) There is $S \subseteq T^{p^{*}} \uparrow\left[V_{\kappa}\right]^{n}$ such that

(1.1) $\forall k<n \exists \xi<1(\bar{E}) \operatorname{Suc}_{S}\left(\left\langle\bar{\nu}_{1}, \ldots, \bar{\nu}_{k}\right\rangle\right) \in E_{\mathrm{mc}\left(p^{*}\right)}(\xi)$,

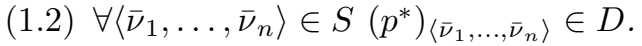

(2) $\forall\left\langle\bar{\nu}_{1}, \ldots, \bar{\nu}_{n}\right\rangle \in T^{p^{*}} \forall q \leq^{*}\left(p^{*}\right)_{\left\langle\bar{\nu}_{1}, \ldots, \bar{\nu}_{n}\right\rangle} q \notin D$.

Proof. We give the proof for $n=1$. Adapting the proof for higher $n$ 's requires that whenever we enumerate singletons we should enumerate $n$-tuples and when we use $j$ we should use $j_{n}$.

We start an induction on $\xi$ in which we build

$$
\left\langle\bar{\alpha}^{\xi}, u^{\xi} \mid \xi<\kappa\right\rangle \text {. }
$$

We start by setting

$$
\begin{aligned}
u^{0} & =p_{0} \backslash\left\{T^{p_{0}}\right\} \\
\bar{\alpha}^{0} & =\operatorname{mc}\left(p_{0}\right), \\
T^{0} & =T^{p_{0}} \uparrow \pi_{\bar{\alpha}^{0}, 0}^{-1}\left\{\bar{\nu} \mid \kappa^{0}(\bar{\nu}) \text { is inaccessible }\right\}
\end{aligned}
$$

and taking an increasing enumeration

$$
\left\{\kappa^{0}(\bar{\nu}) \mid\langle\bar{\nu}\rangle \in T^{0}\right\}=\left\langle\tau_{\xi} \mid \xi<\kappa\right\rangle .
$$

Assume that we have constructed

$$
\left\langle\bar{\alpha}^{\xi}, u^{\xi} \mid \xi<\xi_{0}\right\rangle \text {. }
$$

We have two cases. If $\xi_{0}$ is limit, choose $\bar{\alpha}^{\xi_{0}}>_{\overline{\mathrm{E}}} \bar{\alpha}^{\xi}$ for all $\xi<\xi_{0}$ and set

$$
u^{\xi_{0}}=\bigcup_{\xi<\xi_{0}} u^{\xi} \cup\left\{\left\langle\bar{\alpha}^{\xi_{0}}, t\right\rangle\right\} \text { where } \kappa^{0}(t)=\tau_{\xi_{0}} .
$$

If $\xi_{0}=\xi+1$, for each $\bar{\nu}$ such that $\kappa^{0}(\bar{\nu})=\tau_{\xi}$ we set

$$
S(\bar{\nu})=\left(\prod_{\substack{\bar{\alpha} \in \operatorname{supp}^{\xi} u^{\xi} \\ \kappa\left(\left(u^{\xi}\right)^{\bar{\alpha}}\right)<\tau_{\xi}}}\left\{\bar{\mu} \mid \kappa^{0}(\bar{\mu})=\kappa^{0}(\bar{\nu})\right\}\right) \times\{\langle\bar{\nu}\rangle\} .
$$


Let

$$
S=\bigcup_{\kappa^{0}(\bar{\nu})=\tau_{\xi}} S(\bar{\nu})
$$

and fix an enumeration of $S$,

$$
S=\left\langle s^{\xi_{0}, \rho} \mid \rho<\tau_{\xi_{0}}\right\rangle .
$$

There are fewer than $\tau_{\xi_{0}}$ elements in $S$. We use $\tau_{\xi_{0}}$, because this is the maximum size $S$ can have without "killing" the induction.

We do induction on $\rho$ which builds

$$
\left\langle\bar{\alpha}^{\xi_{0}, \rho}, u_{0}^{\xi_{0}, \rho}, T_{0}^{\xi_{0}, \rho}, u_{1}^{\xi_{0}, \rho}, T_{1}^{\xi_{0}, \rho} \mid \rho<\tau_{\xi_{0}}\right\rangle,
$$

from which we build $\left\langle\bar{\alpha}^{\xi_{0}}, u^{\xi_{0}}\right\rangle$. Set

$$
\begin{aligned}
& \bar{\alpha}^{\xi_{0}, 0}=\bar{\alpha}^{\xi}, \\
& u_{0}^{\xi_{0}, 0}=u_{0}^{\xi} .
\end{aligned}
$$

Assume we have constructed $\left\langle\bar{\alpha}^{\xi_{0}, \rho}, u_{0}^{\xi_{0}, \rho}, T_{0}^{\xi_{0}, \rho} \mid \rho<\rho_{0}\right\rangle$.

We have two cases.

If $\rho_{0}$ is limit, set

$$
\begin{aligned}
& \forall \rho<\rho_{0} \bar{\alpha}^{\xi_{0}, \rho_{0}}>_{\overline{\mathrm{E}}} \bar{\alpha}^{\xi_{0}, \rho}, \\
& u^{\xi_{0}, \rho_{0}}=\bigcup_{\rho<\rho_{0}} u^{\xi_{0}, \rho} \cup\left\{\left\langle\bar{\alpha}^{\xi_{0}, \rho_{0}}, t\right\rangle\right\} \text { where } \kappa^{0}(t)=\tau_{\xi} .
\end{aligned}
$$

We set $T_{0}^{\xi_{0}, \rho_{0}}$ and $T_{1}^{\xi_{0}, \rho_{0}}$ equal to anything we like, because we do not use them later.

$$
\text { If } \begin{aligned}
\rho_{0}=\rho+1, \text { let }\langle\bar{\nu}\rangle= & s^{\xi_{0}, \rho}(2) \text {. Set } \\
& u^{\prime \prime}=u_{1}^{\prime \prime} \frown u_{0}^{\prime \prime}=\left(u_{0}^{\xi_{0}, \rho}\right)_{\left\langle s^{\xi_{0}, \rho}\right\rangle}, \\
& T_{0}^{\prime \prime}=\pi_{\bar{\alpha}^{\xi}, \bar{\alpha}^{0}}^{-1}\left(T_{\langle\bar{\nu}\rangle}^{0}\right), \\
& T_{1}^{\prime \prime}=\pi_{\operatorname{mc}\left(u_{1}^{\prime \prime}\right), \bar{\nu}}^{-1}\left(T^{0}(\bar{\nu})\right) .
\end{aligned}
$$

If there are

$$
\begin{aligned}
& q_{1}^{\prime} \leq^{*} u_{1}^{\prime \prime} \cup\left\{T_{1}^{\prime \prime}\right\}, \\
& q_{0}^{\prime} \leq^{*} u_{0}^{\prime \prime} \cup\left\{T_{0}^{\prime \prime}\right\}
\end{aligned}
$$

such that

$$
q_{1}^{\prime} \frown q_{0}^{\prime} \in D
$$

then set

$$
\begin{aligned}
\bar{\alpha}^{\xi_{0}, \rho_{0}} & =\operatorname{mc}\left(q_{0}^{\prime}\right), \\
u_{0}^{\xi_{0}, \rho_{0}} & =u^{\xi_{0}, \rho} \cup\left(q_{0}^{\prime} \backslash\left(u_{0}^{\prime \prime} \cup\left\{T^{q_{0}^{\prime}}\right\}\right)\right), \\
T_{0}^{\xi_{0}, \rho_{0}} & =T^{q_{0}^{\prime}} \\
u_{1}^{\xi_{0}, \rho_{0}} & =q_{1}^{\prime} \backslash\left(u_{1}^{\prime \prime} \cup\left\{T^{q_{1}^{\prime}}\right\}\right), \\
\bar{\alpha}_{1}^{\xi_{0}, \rho_{0}} & =\operatorname{mc}\left(q_{1}^{\prime}\right), \\
T_{1}^{\xi_{0}, \rho_{0}} & =T^{q_{1}^{\prime}}
\end{aligned}
$$


otherwise set

$$
\begin{aligned}
& \bar{\alpha}^{\xi_{0}, \rho_{0}}=\bar{\alpha}^{\xi_{0}, \rho}, \\
& u_{0}^{\xi_{0}, \rho_{0}}=u^{\xi_{0}, \rho}, \\
& T_{0}^{\xi_{0}, \rho_{0}}=T_{0}^{\prime \prime}, \\
& u_{1}^{\xi_{0}, \rho_{0}}=\emptyset, \\
& \bar{\alpha}_{1}^{\xi_{0}, \rho_{0}}=\operatorname{mc}\left(u^{\prime \prime}\right), \\
& T_{1}^{\xi_{0}, \rho_{0}}=T_{1}^{\prime \prime} .
\end{aligned}
$$

When the induction on $\rho$ terminates we have $\left\langle\bar{\alpha}^{\xi_{0}, \rho}, u_{0}^{\xi_{0}, \rho}, T_{0}^{\xi_{0}, \rho}, u_{1}^{\xi_{0}, \rho}, T_{1}^{\xi_{0}, \rho}\right| \rho<$ $\left.\tau_{\xi_{0}}\right\rangle$. We continue with the induction on $\xi$. We set

$$
\begin{aligned}
& \forall \rho<\tau_{\xi_{0}} \bar{\alpha}^{\xi_{0}}>_{\overline{\mathrm{E}}} \bar{\alpha}^{\xi_{0}, \rho}, \\
& u_{0}^{\xi_{0}}=\bigcup_{\rho<\tau_{\xi_{0}}} u_{0}^{\xi_{0}, \rho} \cup\left\{\left\langle\bar{\alpha}^{\xi_{0}}, t\right\rangle\right\} \text { where } \max \kappa^{0}(t)=\tau_{\xi} .
\end{aligned}
$$

When the induction on $\xi$ terminates we have $\left\langle\bar{\alpha}^{\xi}, u_{0}^{\xi} \mid \xi<\kappa\right\rangle$. Let

$$
\begin{aligned}
& \forall \xi<\kappa \bar{\alpha}^{* \prime}>_{\overline{\mathrm{E}}} \bar{\alpha}^{\xi}, \\
& p_{0}^{* \prime}=\bigcup_{\xi<\kappa} u_{0}^{\xi} \cup\left\{\left\langle\bar{\alpha}^{* \prime}, t\right\rangle\right\} \text { where } \max \kappa^{0}(t)=\max p_{0}^{0} .
\end{aligned}
$$

We set

$$
\operatorname{Lev}_{0}\left(T^{p_{0}^{* \prime}}\right)=\pi_{\bar{\alpha}^{* \prime}, \bar{\alpha}^{0}}^{-1} \operatorname{Lev}_{0}\left(T^{0}\right)
$$

Let us consider $\langle\bar{\nu}\rangle \in \operatorname{Lev}_{0}\left(T^{p_{0}^{* \prime}}\right)$. There is $\xi$ such that $\kappa^{0}(\bar{\nu})=\tau_{\xi}$. We set

$$
\begin{aligned}
& s(1)=\left\{\left\langle\bar{\alpha}, \pi_{\bar{\alpha}^{* \prime}, \bar{\alpha}}(\bar{\nu})\right\rangle \mid \bar{\alpha} \in \operatorname{supp} p_{0}^{*}\right\}, \\
& s(2)=\left\{\left\langle\pi_{\bar{\alpha}^{* \prime}, \bar{\alpha}^{0}}(\bar{\nu})\right\rangle\right\} .
\end{aligned}
$$

Let $\xi_{0}=\xi+1$. By our construction there is $\rho$ such that

$$
\left.\left(u_{0}^{\xi_{0}, \rho_{0}}\right)_{\langle s\rangle}=\left(u_{0}^{\xi_{0}, \rho_{0}}\right)_{\langle s} \xi_{0}, \rho\right\rangle
$$

where $\rho_{0}=\rho+1$. We set

$$
\begin{aligned}
& T_{\langle\bar{\nu}\rangle}^{p_{0}^{* \prime}}=\pi_{\bar{\alpha}^{* \prime}, \bar{\alpha}^{\xi_{0}, \rho_{0}}}^{-1}\left(T^{\xi_{0}, \rho_{0}}\right) \cap \pi_{\bar{\alpha}^{* \prime}, \bar{\alpha}^{0}}^{-1}\left(T_{\left\langle\pi_{\bar{\alpha}^{*}, \bar{\alpha}^{0}}(\bar{\nu})\right\rangle}^{0}\right), \\
& T^{p_{0}^{* \prime}}(\bar{\nu})=T_{1}^{\xi_{0}, \rho_{0}}, \\
& \bar{\alpha}_{1}^{\prime}(\bar{\nu})=\bar{\alpha}_{1}^{\xi_{0}, \rho_{0}}, \\
& p_{1}^{\prime}(\bar{\nu})=u_{1}^{\xi_{0}, \rho_{0}} \text {. }
\end{aligned}
$$

Note that $T^{p_{0}^{* \prime}}$ is not legal as a tree in a condition, because if $p_{1}^{\prime}(\bar{\nu}) \neq \emptyset$, then $T^{p_{0}^{* \prime}}(\bar{\nu})$ might be defined on too high a coordinate. We abuse the notation, because wherever $T^{p_{0}^{* \prime}}(\bar{\nu})$ appears, so does $p_{1}^{\prime}(\bar{\nu})$. 
Let us show that $p_{0}^{* \prime}$ approximates the $p^{*}$ we are looking for. So let $\langle\bar{\nu}\rangle \in T^{p_{0}^{* \prime}}$ and assume

$$
\begin{aligned}
& q_{1}^{\prime} \leq^{*} p_{1}^{\prime}(\bar{\nu}) \cup\left(\left(p_{0}^{* \prime}\right)_{\langle\bar{\nu}\rangle}\right)_{1}, \\
& q_{0}^{\prime} \leq^{*}\left(\left(p_{0}^{* \prime}\right)_{\langle\bar{\nu}\rangle}\right)_{0}, \\
& q_{1}^{\prime} \frown q_{0}^{\prime} \in D .
\end{aligned}
$$

Let $\xi$ be such that $\kappa^{0}(\bar{\nu})=\tau_{\xi}$. Set

$$
\begin{aligned}
& s(1)=\left\{\left\langle\bar{\alpha}, \pi_{\bar{\alpha}^{* \prime}, \bar{\alpha}}(\bar{\nu})\right\rangle \mid \bar{\alpha} \in \operatorname{supp} p_{0}^{*}\right\}, \\
& s(2)=\left\{\left\langle\pi_{\bar{\alpha}^{* \prime}, \bar{\alpha}^{0}}(\bar{\nu})\right\rangle\right\},
\end{aligned}
$$

where $\xi_{0}=\xi+1, \rho_{0}=\rho+1$. By our construction there is a $\rho$ such that

$$
\left(u^{\xi_{0}, \rho_{0}}\right)_{\left\langle s^{\left.\xi_{0}, \rho\right\rangle}\right.}=\left(u^{\xi_{0}, \rho_{0}}\right)_{\langle s\rangle} \text {. }
$$

Let us set

$$
r=\left(\left(u_{\left\langle s \xi_{0}, \rho\right\rangle}^{\xi_{0}, \rho_{0}}\right)_{1} \cup\left\{T_{1}^{\xi_{0}, \rho_{0}}\right\}\right) \frown\left(\left(u_{\left\langle s \xi_{0}, \rho\right\rangle}^{\xi_{0}, \rho_{0}}\right)_{0} \cup\left\{T_{0}^{\xi_{0}, \rho_{0}}\right\}\right) .
$$

By construction we have

$$
\left(p_{1}^{\prime}(\bar{\nu}) \cup\left(\left(p_{0}^{* \prime}\right)_{\langle\bar{\nu}\rangle}\right)_{1}\right) \frown\left(\left(p_{0}^{* \prime}\right)_{\langle\bar{\nu}\rangle}\right)_{0} \leq^{*} r .
$$

So what we have is

$$
D \ni q_{1}^{\prime} \frown q_{0}^{\prime} \leq^{*} r .
$$

This is a positive answer to the question in the induction. Hence

$$
r \in D,
$$

which gives us, by openness of $D$, that

$$
\left(p_{1}^{\prime}(\bar{\nu}) \cup\left(\left(p_{0}^{* \prime}\right)_{\langle\bar{\nu}\rangle}\right)_{1}\right) \frown\left(\left(p_{0}^{* \prime}\right)_{\langle\bar{\nu}\rangle}\right)_{0} \in D .
$$

Having proved this approximation property of $p_{0}^{* \prime}$, let us consider the set

$$
B=\left\{\langle\bar{\nu}\rangle \in T^{p_{0}^{* \prime}} \mid \exists q \leq^{*}\left(p_{1}^{\prime}(\bar{\nu}) \cup\left(\left(p_{0}^{* \prime}\right)_{\langle\bar{\nu}\rangle}\right)_{1}\right) \frown\left(\left(p_{0}^{* \prime}\right)_{\langle\bar{\nu}\rangle}\right)_{0} q \in D\right\} .
$$

Let $\bar{\alpha}^{* \prime}=\operatorname{mc}\left(p_{0}^{* \prime}\right)$. There are two cases to be considered.

(1) $\exists \zeta<\mathrm{l}(\bar{E}) B \in E_{\bar{\alpha}^{* \prime}}(\zeta)$.

Let us set

$$
\begin{aligned}
& p_{1}^{\zeta}=j\left(p_{1}^{\prime}\right)\left(\bar{E}_{\bar{\alpha}^{* \prime}}\lceil\zeta)\right. \\
& \bar{\beta}^{\zeta \prime}=\operatorname{mc}\left(p_{1}^{\zeta}\right) \\
& A^{\zeta}=\left\{\langle\bar{\nu}\rangle \mid\left(\left(p_{1}^{\zeta}\right)_{\langle\bar{\nu}\rangle}\right)_{1}=p_{1}^{\prime}\left(\pi_{\bar{\beta}^{\zeta^{\prime}}, \bar{\alpha}^{* \prime}}(\bar{\nu})\right)\right\}
\end{aligned}
$$

Clearly

$$
A^{\zeta} \in E_{\bar{\beta}^{\zeta \prime}}(\zeta) .
$$

Let $\bar{\beta}^{\zeta}>_{\overline{\mathrm{E}}} \bar{\beta}^{\zeta \prime}, \bar{\alpha}^{* \prime}$. Set $T^{\zeta}=\pi_{\bar{\beta}^{\zeta}, \bar{\alpha}^{* \prime}}^{-1}\left(T^{p_{0}^{* \prime}}\right)$. Since $T^{p_{0}^{* \prime}}$ is not legal as a tree, we give the necessary modification making $T^{\zeta}$ legal:

$$
\begin{aligned}
& \forall \bar{\nu} \in \pi_{\bar{\beta}^{\zeta}, \bar{\beta}^{\zeta \prime}}^{-1}\left(A^{\zeta}\right) T^{\zeta}(\bar{\nu})=\pi_{\bar{\nu}, \bar{\alpha}_{1}^{\prime}\left(\pi_{\bar{\beta} \zeta, \bar{\alpha}^{* \prime}}(\bar{\nu})\right)}^{-1} T^{p_{0}^{* \prime}}\left(\pi_{\bar{\beta}^{\zeta}, \bar{\alpha}^{* \prime}}(\bar{\nu})\right), \\
& \forall \bar{\nu} \notin \pi_{\bar{\beta}^{\zeta}, \bar{\beta}^{\zeta \prime}}^{-1}\left(A^{\zeta}\right) T^{\zeta}(\bar{\nu})=\pi_{\bar{\nu}, \bar{\alpha}_{1}^{\prime}\left(\pi_{\bar{\beta} \zeta, \bar{\alpha}^{* \prime}}(\bar{\nu})\right)}^{-1} T^{p_{0}}\left(\pi_{\bar{\beta}^{\zeta}, \bar{\alpha}^{* \prime}}(\bar{\nu})\right), \\
& p^{\zeta}=p_{1}^{\zeta} \cup p_{0}^{* \prime} \cup\left\{\left\langle\beta^{\zeta}, t\right\rangle\right\} \cup\left\{T^{\zeta}\right\} \text { where } \max t^{0}=p_{0}^{0} .
\end{aligned}
$$


The nice property of $p^{\zeta}$ is that when $\langle\bar{\nu}\rangle \in T^{\zeta} \uparrow\left(\pi_{\bar{\beta}^{\zeta}, \bar{\beta}^{\zeta \prime}}\right)^{-1}\left(A^{\zeta}\right)$ we get

$$
\begin{aligned}
\left(p^{\zeta}\right)_{\langle\bar{\nu}\rangle} \leq^{*}\left(p_{1}^{\prime}\left(\pi_{\bar{\beta}^{\zeta}, \bar{\alpha}^{* \prime}}(\bar{\nu})\right)\right. & \left.\cup\left(\left(p_{0}^{* \prime}\right)_{\left\langle\pi_{\bar{\beta} \zeta, \bar{\alpha}^{* \prime}}(\bar{\nu})\right\rangle}\right)_{1}\right) \\
& \frown\left(\left(p_{0}^{* \prime}\right)_{\left\langle\pi_{\bar{\beta}^{\zeta}, \bar{\alpha}^{* \prime}}(\bar{\nu})\right\rangle}\right)_{0} .
\end{aligned}
$$

We set

$$
\begin{aligned}
& p^{*}=p^{\zeta}, \\
& A=\operatorname{Lev}_{0}\left(T^{\zeta}\right) \cap\left(\pi_{\bar{\beta}^{\zeta}, \bar{\beta}^{\zeta \prime}}\right)^{-1} A^{\zeta},
\end{aligned}
$$

and show that the claim is satisfied. Assume that

$$
\begin{aligned}
& \langle\bar{\nu}\rangle \in A, \\
& q_{1}^{\prime} \leq^{*}\left(\left(p^{*}\right)_{\langle\bar{\nu}\rangle}\right)_{1}, \\
& q_{0}^{\prime} \leq^{*}\left(\left(p^{*}\right)_{\langle\bar{\nu}\rangle}\right)_{0}, \\
& q_{1}^{\prime} \frown q_{0}^{\prime} \in D .
\end{aligned}
$$

Note that

$$
\begin{aligned}
& \left(\left(p^{*}\right)_{\langle\bar{\nu}\rangle}\right)_{1} \leq^{*} p_{1}^{\prime}\left(\pi_{\bar{\beta}^{\zeta}, \bar{\alpha}^{* \prime}}\right) \cup\left(\left(p_{0}^{* \prime}\right)_{\left\langle\pi_{\bar{\beta} \zeta, \bar{\alpha}^{* \prime}}(\bar{\nu})\right\rangle}\right)_{1}, \\
& \left(\left(p^{*}\right)_{\langle\bar{\nu}\rangle}\right)_{0} \leq^{*}\left(\left(p_{0}^{* \prime}\right)_{\left\langle\pi_{\bar{\beta}^{\zeta}, \bar{\alpha}^{* \prime}}(\bar{\nu})\right\rangle}\right)_{0} .
\end{aligned}
$$

Hence, we know that

$$
\begin{aligned}
& q_{1}^{\prime} \leq^{*} p_{1}^{\prime}\left(\pi_{\bar{\beta}^{\zeta}, \bar{\alpha}^{* \prime}}(\bar{\nu})\right) \cup\left(\left(p_{0}^{* \prime}\right)_{\left\langle\pi_{\bar{\beta} \zeta, \bar{\alpha}^{* \prime}}(\bar{\nu})\right\rangle}\right)_{1}, \\
& q_{0}^{\prime} \leq^{*}\left(\left(p_{0}^{* \prime}\right)_{\left.\left\langle\pi_{\bar{\beta} \zeta, \bar{\alpha}^{* \prime}}(\bar{\nu})\right\rangle\right)_{0},}\right. \\
& q_{1}^{\prime} \frown q_{0}^{\prime} \in D .
\end{aligned}
$$

This is the assumption (6.4.1). So from (6.4.2) we know that

$$
\left(p_{1}^{\prime}\left(\pi_{\bar{\beta}^{\zeta}, \bar{\alpha}^{* \prime}}(\bar{\nu})\right) \cup\left(\left(p_{0}^{* \prime}\right)_{\left\langle\pi_{\bar{\beta} \zeta, \bar{\alpha}^{* \prime}}(\bar{\nu})\right\rangle}\right)_{1}\right) \frown\left(\left(p_{0}^{* \prime}\right)_{\left\langle\pi_{\bar{\beta} \zeta, \bar{\alpha}^{*}}(\bar{\nu})\right\rangle}\right)_{0} \in D .
$$

Hence, by openness of $D$,

$$
\left(p^{*}\right)_{\langle\bar{\nu}\rangle} \in D \text {. }
$$

(2) $\forall \zeta<\mathrm{l}(\bar{E}) B \notin E_{\bar{\alpha}^{* \prime}}(\zeta)$, which is the same as saying that

$\left\{\langle\bar{\nu}\rangle \in T^{p_{0}^{* \prime}} \mid \forall q \leq^{*}\left(p_{1}^{\prime}(\bar{\nu}) \cup\left(\left(p_{0}^{* \prime}\right)_{\langle\bar{\nu}\rangle}\right)_{1}\right)^{\frown}\left(\left(p_{0}^{* \prime}\right)_{\langle\bar{\nu}\rangle}\right)_{0} q \notin D\right\} \in \bar{E}_{\bar{\alpha}^{* \prime}}$.

In fact, from the construction we can see that

$$
\left\{\langle\bar{\nu}\rangle \in T^{p_{0}^{* \prime}} \mid p_{1}^{\prime}(\bar{\nu})=\emptyset\right\} \in \bar{E}_{\bar{\alpha}^{* \prime}} .
$$

So we really have

$$
A=\left\{\langle\bar{\nu}\rangle \in T^{p_{0}^{* \prime}} \mid \forall q \leq^{*}\left(p_{0}^{* \prime}\right)_{\langle\bar{\nu}\rangle} q \notin D\right\} \in \bar{E}_{\bar{\alpha}^{* \prime}},
$$

and the completion is quite easy now; we set

$$
\begin{aligned}
& T^{p^{*}}=T^{p^{* \prime}}\lceil A, \\
& p^{*}=p_{0}^{* \prime} \cup\left\{T^{p^{*}}\right\} .
\end{aligned}
$$

Claim 6.5. Let $D$ be dense open in $P_{\bar{E}} / P_{\bar{\epsilon}}, p=p_{0} \in P_{\bar{E}} / P_{\bar{\epsilon}}$. Then there is $p^{*} \leq^{*} p$ such that one and only one of the following is true:

(1) There are $n<\omega, S \subseteq T^{p^{*}} \uparrow\left[V_{\kappa}\right]^{n}$ such that 


$$
\begin{aligned}
& \text { (1.1) } \forall k<n \exists \xi<\mathrm{l}(\bar{E}) \operatorname{Suc}_{S}\left(\left\langle\bar{\nu}_{1}, \ldots, \bar{\nu}_{k}\right\rangle\right) \in E_{\mathrm{mc}\left(p^{*}\right)}(\xi), \\
&(1.2) \forall\left\langle\bar{\nu}_{1}, \ldots, \bar{\nu}_{n}\right\rangle \in S\left(p^{*}\right)\left\langle\bar{\nu}_{1}, \ldots, \bar{\nu}_{n}\right\rangle \in D . \\
&(2) \forall n<\omega \forall\left\langle\bar{\nu}_{1}, \ldots, \bar{\nu}_{n}\right\rangle \in T^{p^{*} \forall q \leq^{*}\left(p^{*}\right)_{\left\langle\bar{\nu}_{1}, \ldots, \bar{\nu}_{n}\right\rangle} q \notin D .}
\end{aligned}
$$

Proof. Let $p^{0}=p$.

Generate $p^{n+1} \leq^{*} p^{n}$ by invoking Claim 6.4 for $n+1$ levels.

Take $\forall n<\omega p^{*} \leq p^{n}$.

Claim 6.6. Let $D$ be dense open in $P_{\bar{E}} / P_{\bar{\epsilon}}, p=p_{0} \in P_{\bar{E}} / P_{\bar{\epsilon}}$. Then there are $n<\omega, p^{*} \leq^{*} p, S \subseteq T^{p^{*}}\left\lceil\left[V_{\kappa}\right]^{n}\right.$ such that

(1) $\forall k<n \exists \xi<\mathrm{l}(\bar{E}) \operatorname{Suc}_{S}\left(\left\langle\bar{\nu}_{1}, \ldots, \bar{\nu}_{k}\right\rangle\right) \in E_{\mathrm{mc}\left(p^{*}\right)}(\xi)$,

(2) $\forall\left\langle\bar{\nu}_{1}, \ldots, \bar{\nu}_{n}\right\rangle \in S\left(p^{*}\right)_{\left\langle\bar{\nu}_{1}, \ldots, \bar{\nu}_{n}\right\rangle} \in D$.

Proof. Towards a contradiction, let us assume that the conclusion is false. That means that for all $p^{*} \leq^{*} p$, for all $n<\omega$, for all $S \subseteq T^{p^{*}} \uparrow\left[V_{\kappa}\right]^{n}$ such that

$$
\forall k<n \forall\left\langle\bar{\nu}_{1}, \ldots, \bar{\nu}_{k}\right\rangle \in S \exists \xi<1(\bar{E}) \operatorname{Suc}_{S}\left(\left\langle\bar{\nu}_{1}, \ldots, \bar{\nu}_{k}\right\rangle\right) \in E_{\operatorname{mc}\left(p^{*}\right)}(\xi)
$$

we have

$$
\exists\left\langle\bar{\nu}_{1}, \ldots, \bar{\nu}_{n}\right\rangle \in S\left(p^{*}\right)_{\left\langle\bar{\nu}_{1}, \ldots, \bar{\nu}_{n}\right\rangle} \notin D .
$$

We construct $\mathrm{a} \leq^{*}$-decreasing sequence as follows: We set $p^{0}=p$. We construct $p^{n+1}$ from $p^{n}$ using Claim 6.4 for $n+1$ levels. Due to our assumption we get

$$
\forall\left\langle\bar{\nu}_{1}, \ldots, \bar{\nu}_{n}\right\rangle \in T^{p^{n}} \forall q \leq^{*}\left(p^{n}\right)_{\left\langle\bar{\nu}_{1}, \ldots, \bar{\nu}_{n}\right\rangle} q \notin D .
$$

Choosing $p^{* \prime}$ such that $\forall n<\omega p^{* \prime} \leq^{*} p^{n}$, we get

$$
\forall n<\omega \forall\left\langle\bar{\nu}_{1}, \ldots, \bar{\nu}_{n}\right\rangle \in T^{p_{0}^{* \prime}} \forall q \leq^{*}\left(p^{* \prime}\right)_{\left\langle\bar{\nu}_{1}, \ldots, \bar{\nu}_{n}\right\rangle} q \notin D .
$$

Construct a tree $T$ from $T^{p^{* \prime}}$ using Lemma 4.10 Let us call $p^{*}$ the condition $p^{* \prime}$ with $T$ substituted for $T^{p^{* \prime}}$. Now if we have

$$
q \leq p^{*}
$$

then there is $\left\langle\bar{\nu}_{1}, \ldots, \bar{\nu}_{n}\right\rangle \in T^{p^{* \prime}}$ such that

$$
q \leq^{*}\left(p^{* \prime}\right)_{\left\langle\bar{\nu}_{1}, \ldots, \bar{\nu}_{n}\right\rangle} .
$$

Hence

$$
q \notin D .
$$

However, $D$ is dense, a contradiction.

Claim 6.7. Let $D$ be dense open in $P_{\bar{E}}$, and let $p=p_{1} \frown p_{0} \in P_{\bar{E}}$. Then there is $p^{*} \leq^{*} p$ such that

$$
\begin{aligned}
\exists S^{1} \exists n_{1} \forall\left\langle\bar{\nu}_{1,1}, \ldots, \bar{\nu}_{1, n_{1}}\right\rangle \in S^{1} \ldots & \exists S^{0} \exists n_{0} \forall\left\langle\bar{\nu}_{0,1}, \ldots, \bar{\nu}_{0, n_{0}}\right\rangle \in S^{0} \\
& \left(p_{1}^{*}\right)_{\left\langle\bar{\nu}_{1,1}, \ldots, \bar{\nu}_{1, n_{1}}\right\rangle} \frown \ldots \frown\left(p_{0}^{*}\right)_{\left\langle\bar{\nu}_{0,1}, \ldots, \bar{\nu}_{0, n_{0}}\right\rangle} \in D
\end{aligned}
$$

where

(1) $S^{i} \subseteq T^{p_{i}^{*}} \uparrow\left[V_{\kappa}\right]^{n_{i}}$

(2) $\forall l<n_{i} \forall\left\langle\bar{\nu}_{1}, \ldots, \bar{\nu}_{l}\right\rangle \in S^{i} \exists \xi \operatorname{Suc}_{S^{i}}\left(\left\langle\bar{\nu}_{1}, \ldots, \bar{\nu}_{l}\right\rangle\right) \in E_{\mathrm{mc}\left(p_{i}^{*}\right)}(\xi)$.

Proof. Let $\bar{\epsilon}$ be such that $p_{1} \in P_{\bar{\epsilon}}$. We prove that there are $n<\omega, p_{0}^{*} \leq^{*} p_{0}$, $q_{1} \leq p_{1}, S \subseteq T^{p^{*}} \uparrow\left[V_{\kappa}\right]^{n}$ such that

(1) $\forall k<n \exists \xi<1(\bar{E}) \operatorname{Suc}_{S}\left(\left\langle\bar{\nu}_{1}, \ldots, \bar{\nu}_{k}\right\rangle\right) \in E_{\mathrm{mc}\left(p^{*}\right)}(\xi)$, 
(2) $\forall\left\langle\bar{\nu}_{1}, \ldots, \bar{\nu}_{n}\right\rangle \in S q_{1} \frown\left(p^{*}\right)_{\left\langle\bar{\nu}_{1}, \ldots, \bar{\nu}_{n}\right\rangle} \in D$.

Set

$$
E=\left\{r \in P_{\bar{E}} \mid \exists q_{1} q_{1} \frown r \in D, q_{1} \leq p_{1}\right\} .
$$

This $E$ is dense open in $P_{\bar{E}} / P_{\bar{\epsilon}}$ : Let $r \in P_{\bar{E}} / P_{\bar{\epsilon}}$. Then $p_{1} \frown r \in P_{\bar{E}}$. By density of $D$, there is $q_{1} \frown s \in D$ such that $q_{1} \leq p_{1}, s \leq r$. By the definition of $E, s \in E$. Hence $E$ is dense. Openness of $E$ is immediate from the openness of $D$.

By Claim 6.6 there are $p_{0}^{*} \leq^{*} p_{0}, S^{\prime}, n<\omega$ such that

(1) $\forall k<n \exists \xi<1(\bar{E}) \operatorname{Suc}_{S^{\prime}}\left(\left\langle\bar{\nu}_{1}, \ldots, \bar{\nu}_{k}\right\rangle\right) \in E_{\mathrm{mc}\left(p^{*}\right)}(\xi)$,

(2) $\forall\left\langle\bar{\nu}_{1}, \ldots, \bar{\nu}_{n}\right\rangle \in S^{\prime}\left(p_{0}^{*}\right)_{\left\langle\bar{\nu}_{1}, \ldots, \bar{\nu}_{n}\right\rangle} \in E$.

This means that $\forall\left\langle\bar{\nu}_{1}, \ldots, \bar{\nu}_{n}\right\rangle \in S^{\prime}$ there is $q_{1}\left(\bar{\nu}_{1}, \ldots, \bar{\nu}_{n}\right) \leq p_{1}$ such that

$$
\forall\left\langle\bar{\nu}_{1}, \ldots, \bar{\nu}_{n}\right\rangle \in S^{\prime} q_{1}\left(\bar{\nu}_{1}, \ldots, \bar{\nu}_{n}\right) \frown\left(p_{0}^{*}\right)_{\left\langle\bar{\nu}_{1}, \ldots, \bar{\nu}_{n}\right\rangle} \in D .
$$

Since $\left|P_{\bar{\epsilon}}\right|<\kappa, q_{1}\left(\bar{\nu}_{1}, \ldots, \bar{\nu}_{n}\right)$ is in fact almost always constant. Hence, by shrinking $S^{\prime}$ to $S$ and letting $q_{1}$ be this constant value, we get

$$
\forall\left\langle\bar{\nu}_{1}, \ldots, \bar{\nu}_{n}\right\rangle \in S q_{1} \frown\left(p_{0}^{*}\right)_{\left\langle\bar{\nu}_{1}, \ldots, \bar{\nu}_{n}\right\rangle} \in D .
$$

With this, we have finished the first part of the proof. We use this claim for all conditions in $P_{\bar{\epsilon}}$.

Let $P_{\bar{\epsilon}}=\left\{p_{1}^{\zeta} \mid \zeta<\lambda\right\}$ where $\lambda<\kappa$.

We construct by induction a $\leq^{*}$-decreasing sequence $\left\langle p_{0}^{\zeta} \mid \zeta<\lambda\right\rangle$. Set

$$
p_{0}^{0}=p_{0} .
$$

Assume we have constructed $\left\langle p_{0}^{\zeta} \mid \zeta<\zeta_{0}\right\rangle$.

If $\zeta_{0}$ is limit, choose $p_{0}^{\zeta_{0}} \leq * p_{0}^{\zeta}$ for all $\zeta<\zeta_{0}$.

If $\zeta_{0}=\zeta+1$, use the first part of the proof on $p_{1}^{\zeta} \frown p_{0}^{\zeta}$ to construct $p_{0}^{\zeta_{0}}$.

When the induction terminates we have $\left\langle p_{0}^{\zeta} \mid \zeta<\lambda\right\rangle$. Choose

$$
\forall \zeta<\lambda p_{0}^{*} \leq^{*} p_{0}^{\zeta}
$$

Let

$$
D_{\bar{\epsilon}}=\left\{q_{1} \in P_{\bar{\epsilon}} \mid \exists n \exists S \forall\left\langle\bar{\nu}_{1}, \ldots, \bar{\nu}_{n}\right\rangle \in S q_{1} \frown\left(p_{0}^{*}\right)_{\left\langle\bar{\nu}_{1}, \ldots, \bar{\nu}_{n}\right\rangle} \in D\right\} .
$$

$D_{\bar{\epsilon}}$ is dense open: Let $q_{1} \in P_{\bar{\epsilon}}$. Then there is $\zeta$ such that $q_{1}=p_{1}^{\zeta}$. By the induction we have that there are $n, S, r_{1} \leq q_{1}$ such that

$$
\forall\left\langle\bar{\nu}_{1}, \ldots, \bar{\nu}_{n}\right\rangle \in S r_{1} \frown\left(p_{0}^{\zeta_{0}}\right)_{\left\langle\bar{\nu}_{1}, \ldots, \bar{\nu}_{n}\right\rangle} \in D .
$$

By the openness of $D$ we get

$$
\forall\left\langle\bar{\nu}_{1}, \ldots, \bar{\nu}_{n}\right\rangle \in S r_{1} \frown\left(p_{0}^{*}\right)_{\left\langle\bar{\nu}_{1}, \ldots, \bar{\nu}_{n}\right\rangle} \in D .
$$

Hence

$$
r_{1} \in D_{\bar{\epsilon}} .
$$

Since $D_{\bar{\epsilon}}$ is dense open, we can use Claim 6.6. Hence there are $p_{1}^{*} \leq p_{1}, S^{1}, n_{1}$ such that

$$
\forall\left\langle\bar{\nu}_{1}, \ldots, \bar{\nu}_{n_{1}}\right\rangle \in S^{1}\left(p_{1}^{*}\right)_{\left\langle\bar{\nu}_{1}, \ldots, \bar{\nu}_{n_{1}}\right\rangle} \in D_{\bar{\epsilon}} .
$$


This means that

$$
\begin{aligned}
\forall\left\langle\bar{\nu}_{1,1}, \ldots, \bar{\nu}_{1, n_{1}}\right\rangle \in S^{1} \exists S \exists n \forall\left\langle\bar{\nu}_{0,1}, \ldots, \bar{\nu}_{0, n}\right\rangle \in S \\
\left(p_{1}^{*}\right)_{\left\langle\bar{\nu}_{1,1}, \ldots, \bar{\nu}_{1, n_{1}}\right\rangle} \frown\left(p_{0}^{*}\right)_{\left\langle\bar{\nu}_{0,1}, \ldots, \bar{\nu}_{0, n}\right\rangle} \in D,
\end{aligned}
$$

which is what we need to prove.

Finally, we add the last touch.

Proof of Theorem 6.1. The proof is done by induction on $k$. The case $k=1$ is Claim 6.7 We assume, then, that the theorem is proved for $k$ and prove it for $k+1$.

Then $p=p_{k+1} \frown p_{k} \frown \ldots \frown p_{0}$. Let $\bar{\epsilon}$ be such that $p_{k+1} \in P_{\bar{\epsilon}}$. We just repeat the proof of Claim [6.7 with $P_{\bar{\epsilon}}$ and use the induction hypotheses to conclude the proof.

\section{PRIKRY'S CONDITION}

Theorem 7.1. Let $p \in P_{\bar{E}}$, and let $\sigma$ be a formula in the forcing language. Then there is a $p^{*} \leq^{*} p$ such that $p^{*} \| \sigma$.

Proof. The set $\left\{q \in P_{\bar{E}} \mid q \| \sigma\right\}$ is dense open. Assuming $p=p_{k} \frown \ldots \frown p_{0}$ and using [6.1, we get that there is a $q \leq^{*} p$ such that

$$
\begin{aligned}
\exists S^{k} \exists n_{k} \forall\left\langle\bar{\nu}_{k, 1}, \ldots, \bar{\nu}_{k, n_{k}}\right\rangle \in S^{k} \ldots & \exists S^{0} \exists n_{0} \forall\left\langle\bar{\nu}_{0,1}, \ldots, \bar{\nu}_{0, n_{0}}\right\rangle \in S^{0} \\
& \left(p_{k}^{*}\right)_{\left\langle\bar{\nu}_{k, 1}, \ldots, \bar{\nu}_{k, n_{k}}\right\rangle} \frown \ldots \frown\left(p_{0}^{*}\right)_{\left\langle\bar{\nu}_{0,1}, \ldots, \bar{\nu}_{0, n_{0}}\right\rangle} \| \sigma .
\end{aligned}
$$

Recall that we really should write

$$
\begin{aligned}
& S^{k-1}\left(\bar{\nu}_{k, 1}, \ldots, \bar{\nu}_{k, n_{k}}\right), \\
& S^{k-2}\left(\bar{\nu}_{k, 1}, \ldots, \bar{\nu}_{k, n_{k}}, \bar{\nu}_{k-1,1}, \ldots, \bar{\nu}_{k-1, n_{k}}\right), \\
& \vdots
\end{aligned}
$$

In order to avoid (too much) clutter, we use the following convention in the proof. When we write

$$
\vec{\nu} \in \prod_{1 \leq l \leq k} S^{l}
$$

we mean that

$$
\begin{aligned}
& \left\langle\bar{\nu}_{k, 1}, \ldots, \bar{\nu}_{k, n_{k}}\right\rangle \in S^{k}, \\
& \vdots \\
& \left\langle\bar{\nu}_{1,1}, \ldots, \bar{\nu}_{1, n_{1}}\right\rangle \in S^{1},
\end{aligned}
$$

and $r(\vec{\nu})$ is

$$
\left(q_{k}\right)_{\left\langle\bar{\nu}_{k, 1}, \ldots, \bar{\nu}_{k, n_{k}}\right\rangle} \frown \ldots \frown\left(q_{1}\right)_{\left\langle\bar{\nu}_{1,1}, \ldots, \bar{\nu}_{1, n_{1}}\right\rangle} \cdot
$$

We start by naming $q_{0}$ as $q_{0}^{n_{0}}$ and $T^{q_{0}}$ as $T^{0, n_{0}}$. For $\left\langle\bar{\nu}_{1}, \ldots, \bar{\nu}_{n_{0}-1}\right\rangle \in S^{0}$ set

$$
\begin{aligned}
& A^{0}=\left\{\left\langle\bar{\nu}_{n_{0}}\right\rangle \in \operatorname{Suc}_{T^{0, n_{0}}}\left(\left\langle\bar{\nu}_{1}, \ldots, \bar{\nu}_{n-1}\right\rangle\right) \mid r(\vec{\nu}) \frown\left(q_{0}^{n_{0}}\right)_{\left\langle\bar{\nu}_{1}, \ldots, \bar{\nu}_{n_{0}}\right\rangle} \Vdash \sigma\right\}, \\
& A^{1}=\left\{\left\langle\bar{\nu}_{n_{0}}\right\rangle \in \operatorname{Suc}_{T^{0, n_{0}}}\left(\left\langle\bar{\nu}_{1}, \ldots, \bar{\nu}_{n_{0}-1}\right\rangle\right) \mid r(\vec{\nu}) \frown\left(q_{0}^{n_{0}}\right)_{\left\langle\bar{\nu}_{1}, \ldots, \bar{\nu}_{n_{0}}\right\rangle} \Vdash \neg \sigma\right\} .
\end{aligned}
$$


Note that

$$
\begin{aligned}
& \operatorname{Suc}_{S^{0}}\left(\left\langle\bar{\nu}_{1}, \ldots, \bar{\nu}_{n_{0}-1}\right\rangle\right) \subseteq A^{0} \cup A^{1}, \\
& A^{0} \cap A^{1}=\emptyset .
\end{aligned}
$$

Hence, there is a $\xi<1(\bar{E})$ such that one and only one of the following is true:

(1) $A^{0} \in E_{\operatorname{mc}\left(q_{0}^{n_{0}}\right)}(\xi)$,

(2) $A^{1} \in E_{\operatorname{mc}\left(q_{0}^{n_{0}}\right)}(\xi)$.

In either case, using Lemma 4.8 we can shrink $T_{\left\langle\bar{\nu}_{1}, \ldots, \bar{\nu}_{n_{0}-1}\right\rangle}^{0, n_{0}}$ and get a condition $q_{0}^{\prime}$ such that $r(\vec{\nu}) \frown q_{\left\langle\bar{\nu}_{1}, \ldots, \bar{\nu}_{n_{0}-1}\right\rangle}^{\prime} \| \sigma$.

So now we shrink $T_{\left\langle\bar{\nu}_{1}, \ldots, \bar{\nu}_{n_{0}-1}\right\rangle}^{0, n_{0}}$ for all $\left\langle\bar{\nu}_{1}, \ldots, \bar{\nu}_{n_{0}-1}\right\rangle \in S^{0}$, and we call this tree $T^{0, n_{0}-1}$. The name of the condition $q_{0}^{n_{0}}$ with $T^{0, n_{0}-1}$ substituted for $T^{0, n_{0}}$ is $q_{0}^{n_{0}-1}$. $q_{0}^{n_{0}-1}$ satisfies

$$
\forall\left\langle\bar{\nu}_{1}, \ldots, \bar{\nu}_{n_{0}-1}\right\rangle \in S^{0} r(\vec{\nu}) \frown\left(q_{0}^{n_{0}-1}\right)_{\left\langle\bar{\nu}_{1}, \ldots, \bar{\nu}_{n_{0}-1}\right\rangle} \| \sigma .
$$

We are now in the same position as we were when setting $q_{0}^{n_{0}}$. So by repeating the above arguments we get

$$
p_{0} \geq^{*} q_{0}=q_{0}^{n_{0}} \geq^{*} q_{0}^{n_{0}-1} \geq^{*} \cdots \geq^{*} q_{0}^{1} \geq^{*} q_{0}^{0}
$$

such that for each $l=n_{0}, n_{0}-1, \ldots, 1,0$,

$$
\forall\left\langle\bar{\nu}_{1}, \ldots, \bar{\nu}_{l}\right\rangle \in S^{0} r(\vec{\nu}) \frown\left(q_{0}^{l}\right)_{\left\langle\bar{\nu}_{1}, \ldots, \bar{\nu}_{l}\right\rangle} \| \sigma .
$$

Specifically, we get

$$
r(\vec{\nu}) \frown\left(q_{0}^{0}\right) \| \sigma .
$$

Of course $q_{0}^{0}$ depends on $\vec{\nu}$. Note that we got from $q_{0}^{n_{0}}$ to $q_{0}^{0}$ only by shrinking the trees. So, we repeat this process for all $\vec{\nu}$, calling the resulting condition $q_{0}^{0}(\vec{\nu})$. So we have

$$
\forall \vec{\nu} \in \prod_{1 \leq l \leq k} S^{l} r(\vec{\nu}) \frown q_{0}^{0}(\vec{\nu}) \| \sigma .
$$

By setting

$$
T^{p_{0}^{*}}=\bigcap_{\vec{\nu}} T^{q_{0}^{0}(\vec{\nu})}
$$

and letting $p_{0}^{*}$ be $q_{0}$ with $T^{p_{0}^{*}}$ substituted for $T^{q_{0}}$, we get

$$
\forall \vec{\nu} \in \prod_{1 \leq l \leq k} S^{l} r(\vec{\nu}) \frown p_{0}^{*} \| \sigma .
$$

We are in the same position as in the beginning of the proof. So in the same way we can generate $p_{1}^{*}$ from $p_{1}$ and so on, until we have

$$
p_{k}^{*} \frown \ldots \frown p_{0}^{*} \| \sigma .
$$




\section{Properness}

The notions of $\langle N, P\rangle$-generic and properness, as defined by Shelah [9], are as follows:

Definition 8.1. Let $N \prec H_{\chi}$ be such that

(1) $|N|=\omega$,

(2) $P \in N$.

Then $p \in P$ is called $\langle N, P\rangle$-generic if

$$
p \Vdash\ulcorner\forall D \in \widehat{N} D \text { is dense open in } \widehat{P} \Longrightarrow D \cap \widetilde{G} \cap \widehat{N} \neq \emptyset\urcorner .
$$

Definition 8.2. A forcing notion $P$ is called proper if for all $N \prec H_{\chi}$ such that

(1) $|N|=\omega$,

(2) $P \in N$,

and for all $q \in P \cap N$ there is a $p \leq q$ that is $\langle N, P\rangle$-generic.

We adapt these definitions for our needs (namely, larger submodels), keeping the original names:

Definition 8.3. Let $N \prec H_{\chi}$ be such that

(1) $|N|=\kappa$,

(2) $N \supseteq V_{\kappa}$,

(3) $N \supseteq N^{<\kappa}$,

(4) $P \in N$.

Then $p \in P$ is called $\langle N, P\rangle$-generic if

$$
p \Vdash\ulcorner\forall D \in \widehat{N} D \text { is dense open in } \widehat{P} \Longrightarrow D \cap \widetilde{G} \cap \widehat{N} \neq \emptyset\urcorner .
$$

Definition 8.4. A forcing notion $P$ is called proper if for all $N \prec H_{\chi}$ such that

(1) $|N|=\kappa$,

(2) $N \supseteq V_{\kappa}$,

(3) $N \supseteq N^{<\kappa}$,

(4) $P \in N$,

and for all $q \in P \cap N$ there is a $p \leq q$ that is $\langle N, P\rangle$-generic.

It was brought to our attention by the referee that Woodin initiated the use of proper forcing arguments to show cardinal preservation in Radin-style forcing.

Claim 8.5. Let $p \in P_{\bar{E}}$ and $N \prec H_{\chi}$ be such that

(1) $|N|=\kappa$,

(2) $N \supseteq V_{\kappa}$,

(3) $N \supseteq N^{<\kappa}$,

(4) $P_{\bar{E}} \in N$,

(5) $p \in P_{\bar{E}} \cap N$.

Then there is $p^{*} \leq^{*} p$ such that $p^{*}$ is $\left\langle N, P_{\bar{E}}\right\rangle$-generic.

Proof. Let $p=p_{k(p)} \frown \ldots \frown p_{1} \frown p_{0}$.

Let $\left\langle D_{\xi} \mid \xi<\kappa\right\rangle$ be an enumeration of all dense open subsets of $P_{\bar{E}}$ that are in $N$. Note that for $\xi_{0}<\kappa$ we have that $\left\langle D_{\xi} \mid \xi<\xi_{0}\right\rangle \in N$.

We now start an induction on $\xi$ in which we build

$$
\left\langle\bar{\alpha}^{\xi}, u^{\xi} \mid \xi<\kappa\right\rangle \text {. }
$$


The construction is done ensuring that $\left\langle\bar{\alpha}^{\xi}, u^{\xi} \mid \xi<\xi_{0}\right\rangle \in N$ for all $\xi_{0}<\kappa$. We start by setting

$$
\begin{aligned}
u^{0} & =p_{0} \backslash\left\{T^{p_{0}}\right\} \\
\bar{\alpha}^{0} & =\operatorname{mc}\left(p_{0}\right), \\
T^{0} & =T^{p_{0}}\left\lceil\pi_{\bar{\alpha}^{0}, 0}^{-1}\left\{\bar{\nu} \mid \kappa^{0}(\bar{\nu}) \text { is inaccessible }\right\},\right.
\end{aligned}
$$

and taking an increasing enumeration in $N$,

$$
\left\{\kappa^{0}(\bar{\nu}) \mid\langle\bar{\nu}\rangle \in T^{0}\right\}=\left\langle\tau_{\xi} \mid \xi<\kappa\right\rangle .
$$

Assume then that we have

$$
\left\langle\bar{\alpha}^{\xi}, u^{\xi} \mid \xi<\xi_{0}\right\rangle .
$$

The construction splits now according to whether $\xi_{0}$ is limit or successor. In both cases the work is done inside $N$.

If $\xi_{0}$ is limit, choose $\bar{\alpha}^{\xi_{0}}>_{\overline{\mathrm{E}}} \bar{\alpha}^{\xi}$ for all $\xi<\xi_{0}$ and set

$$
u^{\xi_{0}}=\bigcup_{\xi<\xi_{0}} u^{\xi} \cup\left\{\left\langle\bar{\alpha}^{\xi_{0}}, t\right\rangle\right\} \text { where } \kappa^{0}(t)=\tau_{\xi_{0}} .
$$

If $\xi_{0}=\xi+1$, for each $\bar{\nu}_{1}, \ldots, \bar{\nu}_{n}$ such that $\kappa^{0}\left(\bar{\nu}_{1}\right)<\cdots<\kappa^{0}\left(\bar{\nu}_{n}\right)=\tau_{\xi}$ we set

$$
\begin{aligned}
S\left(\bar{\nu}_{1}, \ldots, \bar{\nu}_{n}\right)= & \left(\prod_{\bar{\alpha} \in \operatorname{supp} u^{\xi}}\left\{\bar{\mu}_{1} \mid \kappa^{0}\left(\bar{\mu}_{1}\right)=\kappa^{0}\left(\bar{\nu}_{1}\right)\right\}\right) \\
& \times\left(\prod_{\bar{\alpha} \in \operatorname{supp} u^{\xi}}\left\{\bar{\mu}_{2} \mid \kappa^{0}\left(\bar{\mu}_{2}\right)=\kappa^{0}\left(\bar{\nu}_{2}\right)\right\}\right) \\
& \vdots \\
& \times\left(\prod_{\bar{\alpha} \in \operatorname{supp} u^{\xi}}\left\{\bar{\mu}_{n} \mid \kappa^{0}\left(\bar{\mu}_{n}\right)=\kappa^{0}\left(\bar{\nu}_{n}\right)\right\}\right) \\
& \times\left\{\left\langle\bar{\nu}_{1}, \ldots, \bar{\nu}_{n}\right\rangle\right\} .
\end{aligned}
$$

Let

$$
S=\bigcup_{\kappa^{0}\left(\bar{\nu}_{1}\right)<\cdots<\kappa^{0}\left(\bar{\nu}_{n}\right)=\tau_{\xi}} S\left(\bar{\nu}_{1}, \ldots, \bar{\nu}_{n}\right)
$$

and fix an enumeration of $S$,

$$
S=\left\langle s^{\xi_{0}, \rho} \mid \rho<\tau_{\xi_{0}}\right\rangle .
$$

We do induction on $\rho$, which builds

$$
\left\langle\bar{\alpha}^{\xi_{0}, \rho}, u_{0}^{\xi_{0}, \rho}, T_{0}^{\xi_{0}, \rho} \mid \rho<\tau_{\xi_{0}}\right\rangle,
$$

from which we build $\left\langle\bar{\alpha}^{\xi_{0}}, u^{\xi_{0}}\right\rangle$. Set

$$
\begin{aligned}
& \bar{\alpha}^{\xi_{0}, 0}=\bar{\alpha}^{\xi}, \\
& u_{0}^{\xi_{0}, 0}=u_{0}^{\xi} .
\end{aligned}
$$

Assume we have constructed $\left\langle\bar{\alpha}^{\xi_{0}, \rho}, u_{0}^{\xi_{0}, \rho}, T_{0}^{\xi_{0}, \rho} \mid \rho<\rho_{0}\right\rangle$. 
If $\rho_{0}$ is limit, set

$$
\begin{aligned}
& \forall \rho<\rho_{0} \bar{\alpha}^{\xi_{0}, \rho_{0}}>_{\overline{\mathrm{E}}} \bar{\alpha}^{\xi_{0}, \rho}, \\
& u^{\xi_{0}, \rho_{0}}=\bigcup_{\rho<\rho_{0}} u^{\xi_{0}, \rho} \cup\left\{\left\langle\bar{\alpha}^{\xi_{0}, \rho_{0}}, t\right\rangle\right\} \text { where } \kappa^{0}(t)=\tau_{\xi_{0}} .
\end{aligned}
$$

We set $T^{\xi_{0}, \rho_{0}}$ equal to anything we like, since we do not use it later.

If $\rho_{0}=\rho+1$, let $s^{\xi_{0}, \rho}(n(s)+1)=\left\langle\bar{\nu}_{1}, \ldots, \bar{\nu}_{n}\right\rangle$ and set

$$
\begin{aligned}
& u^{\prime \prime}=\left(u_{0}^{\xi_{0}, \rho}\right)_{\left\langle s^{\xi_{0}, \rho}\right\rangle}, \\
& T_{0}^{\prime \prime}=\pi_{\bar{\alpha}^{\xi}, \bar{\alpha}^{0}}^{-1}\left(T_{\left\langle\bar{\nu}_{1}, \ldots, \bar{\nu}_{n}\right\rangle}^{0}\right), \\
& T_{1}^{\prime \prime}=\pi_{\mathrm{mc}\left(u_{1}^{\prime \prime}\right), \bar{\nu}_{n}}^{-1}\left(T_{\left\langle\bar{\nu}_{1}, \ldots, \bar{\nu}_{n-1}\right\rangle}^{0}\left(\bar{\nu}_{n}\right)\right), \\
& \vdots \\
& T_{n-1}^{\prime \prime}=\pi_{\mathrm{mc}\left(u_{n-1}^{\prime \prime}\right), \bar{\nu}_{2}}^{-1}\left(T_{\left\langle\bar{\nu}_{1}\right\rangle}^{0}\left(\bar{\nu}_{2}\right)\right), \\
& T_{n}^{\prime \prime}=\pi_{\mathrm{mc}\left(u_{n}^{\prime \prime}\right), \bar{\nu}_{1}}^{-1}\left(T^{0}\left(\bar{\nu}_{1}\right)\right) .
\end{aligned}
$$

Take the enumeration

$$
\begin{aligned}
\left\{D_{\sigma} \mid \sigma<\tau_{\xi}\right\} \times\left\{q \mid q \leq p_{k(p)} \frown \ldots \frown p_{1} \frown u_{n(s)}^{\prime \prime}\right. & \left.\cup\left\{T_{n}^{\prime \prime}\right\} \frown \ldots \frown u_{1}^{\prime \prime} \cup\left\{T_{1}^{\prime \prime}\right\}\right\} \\
& =\left\langle\left\langle E^{\xi_{0}, \rho_{0}, \zeta}, q^{\xi_{0}, \rho_{0}, \zeta}\right\rangle \mid \zeta<\tau_{\xi_{0}}\right\rangle .
\end{aligned}
$$

We start an induction on $\zeta$. Set

$$
\begin{aligned}
& \bar{\alpha}^{\xi_{0}, \rho_{0}, 0}=\bar{\alpha}^{\xi_{0}, \rho}, \\
& u_{0}^{\xi_{0}, \rho_{0}, 0}=u^{\xi_{0}, \rho} .
\end{aligned}
$$

Assume we have constructed $\left\langle\bar{\alpha}^{\xi_{0}, \rho_{0}, \zeta}, u_{0}^{\xi_{0}, \rho_{0}, \zeta}, T_{0}^{\xi_{0}, \rho_{0}, \zeta} \mid \zeta<\zeta_{0}\right\rangle$.

If $\zeta_{0}$ is limit, take

$$
\begin{aligned}
& \forall \zeta<\zeta_{0} \bar{\alpha}^{\xi_{0}, \rho_{0}, \zeta_{0}}>_{\overline{\mathrm{E}}} \bar{\alpha}^{\xi_{0}, \rho_{0}, \zeta}, \\
& u_{0}^{\xi_{0}, \rho_{0}, \zeta_{0}}=\bigcup_{\zeta<\zeta_{0}} u_{0}^{\xi_{0}, \rho_{0}, \zeta} \cup\left\{\left\langle\bar{\alpha}^{\xi_{0}, \rho_{0}, \zeta_{0}}, t\right\rangle\right\} \text { where } \kappa^{0}(t)=\tau_{\xi_{0}} .
\end{aligned}
$$

We set $T^{\xi_{0}, \rho_{0}, \zeta}$ equal to whatever we want, since no use of it is made later.

If $\zeta_{0}=\zeta+1$, we set

$$
\begin{aligned}
& u^{\prime \prime}=\left(u_{0}^{\xi_{0}, \rho_{0}, \zeta}\right)_{s} \xi_{0}, \rho \\
& T_{0}^{\prime \prime}=\pi_{\bar{\alpha}^{\xi_{0}, \rho_{0}, \zeta, \bar{\alpha}^{0}}}^{-1} T_{\left\langle\bar{\nu}_{1}, \ldots, \bar{\nu}_{n}\right\rangle}^{0} .
\end{aligned}
$$

If there is

$$
u_{0}^{\prime} \leq^{*} u_{0}^{\prime \prime} \cup\left\{T_{0}^{\prime \prime}\right\}
$$

such that

$$
q^{\xi_{0}, \rho_{0}, \zeta} \cup u_{0}^{\prime} \in E^{\xi_{0}, \rho_{0}, \zeta},
$$

then set

$$
\begin{aligned}
\bar{\alpha}^{\xi_{0}, \rho_{0}, \zeta_{0}} & =\operatorname{mc}\left(u_{0}^{\prime}\right), \\
u_{0}^{\xi_{0}, \rho_{0}, \zeta_{0}} & =u_{0}^{\xi_{0}, \rho_{0}, \zeta} \cup\left(u_{0}^{\prime} \backslash\left(u_{0}^{\prime \prime} \cup\left\{T^{u_{0}^{\prime}}\right\}\right)\right), \\
T^{\xi_{0}, \rho_{0}, \zeta_{0}} & =T^{u_{0}^{\prime}}
\end{aligned}
$$


otherwise set

$$
\begin{aligned}
\bar{\alpha}^{\xi_{0}, \rho_{0}, \zeta_{0}} & =\bar{\alpha}^{\xi_{0}, \rho_{0}, \zeta}, \\
u_{0}^{\xi_{0}, \rho_{0}, \zeta_{0}} & =u_{0}^{\xi_{0}, \rho_{0}, \zeta}, \\
T_{0}^{\xi_{0}, \rho_{0}, \zeta_{0}} & =T_{0}^{\prime \prime} .
\end{aligned}
$$

When the induction on $\zeta$ terminates, we have $\left\langle\bar{\alpha}^{\xi_{0}, \rho_{0}, \zeta}, u_{0}^{\xi_{0}, \rho_{0}, \zeta}, T^{\xi_{0}, \rho_{0}, \zeta} \mid \zeta<\tau_{\xi_{0}}\right\rangle$.

We continue with the induction on $\rho$. We set

$$
\begin{aligned}
& \forall \zeta<\tau_{\xi_{0}} \bar{\alpha}^{\xi_{0}, \rho_{0}}>_{\overline{\mathrm{E}}} \bar{\alpha}^{\xi_{0}, \rho_{0}, \zeta}, \\
& u_{0}^{\xi_{0}, \rho_{0}}=\bigcup_{\zeta<\tau_{\xi_{0}}} u_{0}^{\xi_{0}, \rho_{0}, \zeta} \cup\left\{\left\langle\bar{\alpha}^{\xi_{0}, \rho_{0}}, t\right\rangle\right\} \text { where } \kappa^{0}(t)=\tau_{\xi_{0}} .
\end{aligned}
$$

When the induction on $\rho$ terminates, we have $\left\langle\bar{\alpha}^{\xi_{0}, \rho}, u_{0}^{\xi_{0}, \rho}, T^{\xi_{0}, \rho} \mid \rho<\tau_{\xi_{0}}\right\rangle$. We continue with the induction on $\xi$. We set

$$
\begin{aligned}
& \forall \rho<\tau_{\xi_{0}} \bar{\alpha}^{\xi_{0}}>_{\overline{\mathrm{E}}} \bar{\alpha}^{\xi_{0}, \rho}, \\
& u_{0}^{\xi_{0}}=\bigcup_{\rho<\tau_{\xi_{0}}} u_{0}^{\xi_{0}, \rho} \cup\left\{\left\langle\bar{\alpha}^{\xi_{0}}, t\right\rangle\right\} \text { where } \kappa^{0}(t)=\tau_{\xi_{0}} .
\end{aligned}
$$

When the induction on $\xi$ terminates we have $\left\langle\bar{\alpha}^{\xi}, u_{0}^{\xi} \mid \xi<\kappa\right\rangle$. We note that this sequence is not in $N$. Let

$$
\begin{aligned}
& \forall \xi<\kappa \bar{\alpha}^{*}>_{\overline{\mathrm{E}}} \bar{\alpha}^{\xi}, \\
& p_{0}^{*}=\bigcup_{\xi<\kappa} u_{0}^{\xi} \cup\left\{\left\langle\bar{\alpha}^{*}, t\right\rangle\right\} \text { where } \kappa^{0}(t)=\max p_{0}^{0} .
\end{aligned}
$$

We construct a series of trees, $R^{n}$, and $T^{p_{0}^{*}}$ is $\bigcap_{n<\omega} R^{n}$. Then

$$
\operatorname{Lev}_{0}\left(R^{0}\right)=\pi_{\bar{\alpha}^{*}, \bar{\alpha}^{0}}^{-1} \operatorname{Lev}_{0}\left(T^{0}\right) .
$$

Let us consider $\left\langle\bar{\nu}_{1}\right\rangle \in \operatorname{Lev}_{0}\left(R^{0}\right)$. There is a $\xi$ such that $\kappa^{0}\left(\bar{\nu}_{1}\right)=\tau_{\xi}$. We set

$$
\begin{aligned}
& s(0)=\left\{\left\langle\bar{\alpha}, \pi_{\bar{\alpha}^{*}, \bar{\alpha}}\left(\bar{\nu}_{1}\right)\right\rangle \mid \bar{\alpha} \in \operatorname{supp} p_{0}^{*}\right\}, \\
& s(1)=\left\{\left\langle\pi_{\left.\left.\bar{\alpha}^{*}, \bar{\alpha}^{0}\left(\bar{\nu}_{1}\right)\right\rangle\right\} .}\right.\right.
\end{aligned}
$$

Let $\xi_{0}=\xi+1$. By our construction there is a $\rho$ such that

$$
\left(u_{0}^{\xi_{0}, \rho_{0}}\right)_{\langle s\rangle}=\left(u_{0}^{\xi_{0}, \rho_{0}}\right)_{\left\langle s^{\left.\xi_{0}, \rho\right\rangle}\right.},
$$

where $\rho_{0}=\rho+1$. We set

$$
R_{\left\langle\bar{\nu}_{1}\right\rangle}^{1}=\pi_{\bar{\alpha}^{*}, \bar{\alpha}^{\xi_{0}, \rho_{0}}}^{-1}\left(T^{\xi_{0}, \rho_{0}}\right) \cap \pi_{\bar{\alpha}^{*}, \bar{\alpha}^{0}}^{-1}\left(T_{\left\langle\pi_{\left.\bar{\alpha}^{*}, \bar{\alpha}^{0}\left(\bar{\nu}_{1}\right)\right\rangle}^{0}\right) .}\right.
$$

Assume that we have constructed $R^{n}$. We set the first $n$ levels of $R^{n+1}$ to be the same as the first $n$ levels of $R^{n}$, and we complete the tree as follows. Let us consider $\left\langle\bar{\nu}_{1}, \ldots, \bar{\nu}_{n}\right\rangle \in R^{n}$. There is a $\xi$ such that $\kappa^{0}\left(\bar{\nu}_{n}\right)=\tau_{\xi}$. We take $s$ as follows:

$$
\begin{aligned}
& \forall 1 \leq k \leq n s(k)=\left\{\left\langle\bar{\alpha}, \pi_{\bar{\alpha}^{*}, \bar{\alpha}}\left(\bar{\nu}_{k}\right)\right\rangle \mid \bar{\alpha} \in \operatorname{supp} p_{0}^{*}\right\}, \\
& s(n+1)=\left\{\left\langle\pi_{\bar{\alpha}^{*}, \bar{\alpha}^{0}}\left(\bar{\nu}_{1}\right), \ldots, \pi_{\bar{\alpha}^{*}, \bar{\alpha}^{0}}\left(\bar{\nu}_{n}\right)\right\rangle\right\} .
\end{aligned}
$$

Let $\xi_{0}=\xi+1$. By our construction there is a $\rho$ such that

$$
\left(u_{0}^{\xi_{0}, \rho_{0}}\right)_{\langle s\rangle}=\left(u_{0}^{\xi_{0}, \rho_{0}}\right)_{\left\langle s \xi_{0}, \rho\right\rangle},
$$


where $\rho_{0}=\rho+1$. We set

$$
R_{\left\langle\bar{\nu}_{1}, \ldots, \bar{\nu}_{n}\right\rangle}^{n+1}=\pi_{\bar{\alpha}^{*}, \bar{\alpha} \xi_{0}, \rho_{0}}^{-1}\left(T^{\xi_{0}, \rho_{0}}\right) \cap \pi_{\bar{\alpha}^{*}, \bar{\alpha}^{0}}^{-1}\left(T_{\left\langle\pi_{\bar{\alpha}^{*}, \bar{\alpha}^{0}}\left(\bar{\nu}_{1}\right), \ldots, \pi_{\bar{\alpha}^{*}, \bar{\alpha}^{0}}\left(\bar{\nu}_{n}\right)\right\rangle}^{0}\right) .
$$

After $\omega$ stages we set

$$
T^{p_{0}^{*}}=\bigcap_{n<\omega} R^{n}
$$

We finish the construction by setting

$$
p^{*}=p_{k(p)} \frown \ldots \frown p_{1} \frown p_{0}^{*} .
$$

We show that $p^{*}$ is as required.

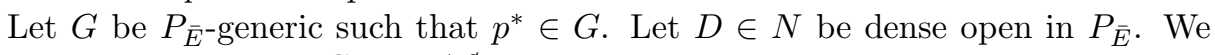
want to show that $D \cap G \cap N \neq \emptyset$.

Choose $q^{\frown} r_{0} \in D \cap G$ such that

$$
\begin{aligned}
& r_{0} \leq^{*} p_{0}^{\prime \prime}, \\
& q \leq p_{k(p)} \frown \ldots \frown p_{1} \frown p_{n}^{\prime \prime} \frown \ldots \frown p_{1}^{\prime \prime},
\end{aligned}
$$

where

$$
\begin{aligned}
& \left\langle\bar{\nu}_{1}, \ldots, \bar{\nu}_{n}\right\rangle \in \operatorname{dom} T^{p_{0}^{*}}, \\
& \kappa^{0}\left(\bar{\nu}_{n}\right)=\tau_{\xi}, \\
& D \in\left\langle D_{\zeta} \mid \zeta<\tau_{\xi}\right\rangle, \\
& p^{\prime \prime}=\left(p_{0}^{*}\right)_{\left\langle\bar{\nu}_{1}, \ldots, \bar{\nu}_{n}\right\rangle} .
\end{aligned}
$$

We take $s$ to be

$$
\begin{aligned}
& \forall 1 \leq k \leq n s(k)=\left\{\left\langle\bar{\alpha}, \pi_{\bar{\alpha}^{*}, \bar{\alpha}}\left(\bar{\nu}_{k}\right)\right\rangle \mid \bar{\alpha} \in \operatorname{supp} p_{0}^{*}\right\}, \\
& s(n+1)=\left\{\left\langle\pi_{\bar{\alpha}^{*}, \bar{\alpha}^{0}}\left(\bar{\nu}_{1}\right), \ldots, \pi_{\bar{\alpha}^{*}, \bar{\alpha}^{0}}\left(\bar{\nu}_{n}\right)\right\rangle\right\} .
\end{aligned}
$$

We get that

$$
\left(p_{0}^{*}\right)_{\left\langle\bar{\nu}_{1}, \ldots, \bar{\nu}_{n}\right\rangle}=\left(p_{0}^{*}\right)_{\langle s\rangle} .
$$

We let $\xi_{0}=\xi+1$. Recall the enumeration of $S$ in the construction. There is a $\rho$ such that

$$
\left(p_{0}^{*} \backslash\left\{\left\langle\operatorname{mc}\left(p_{0}^{*}\right),\left(p_{0}^{*}\right)^{\mathrm{mc}}\right\rangle\right\}\right)_{\langle s\rangle} \cup\left\{\left\langle\operatorname{mc}\left(p_{0}^{*}\right),\left(p_{0}^{*}\right)^{\mathrm{mc}}\right\rangle\right\}=\left(p_{0}^{*}\right)_{\left\langle s^{\xi_{0}, \rho}\right\rangle} .
$$

We let $\rho_{0}=\rho+1$. Considering the construction of $T^{p_{0}^{*}}$, we see that

$$
T_{\left\langle\bar{\nu}_{1}, \ldots, \bar{\nu}_{n}\right\rangle}^{p^{*}} \leq T^{\xi_{0}, \rho_{0}}
$$

hence

$$
\left(p_{0}^{*}\right)_{\langle s\rangle} \leq^{*}\left(u_{0}^{\xi_{0}, \rho_{0}}\right)_{\left\langle s \xi_{0}, \rho_{0}\right\rangle} .
$$

We note that

$$
\forall 1 \leq k \leq n p_{k}^{\prime \prime}=\left(\left(p_{0}^{*}\right)_{\langle s\rangle}\right)_{k} .
$$

Recalling that $q$ was chosen so that

$$
q \leq p_{k(p)} \frown \ldots \frown p_{1} \frown p_{n}^{\prime \prime} \frown \ldots \frown p_{1}^{\prime \prime},
$$

we conclude that there is a $\zeta$ such that $q=q^{\xi_{0}, \rho_{0}, \zeta}$ and $D=E^{\xi_{0}, \rho_{0}, \zeta}$. That is,

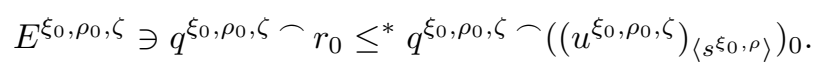


Note that this is an answer to the question we asked in the construction. Hence, because $N$ is elementary, there was such a condition in $N$. Hence

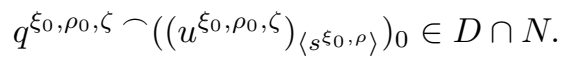

The last point to note is that

$$
q^{\xi_{0}, \rho_{0}, \zeta} \frown\left(\left(u^{\xi_{0}, \rho_{0}, \zeta}\right)_{\left\langle s \xi_{0}, \rho\right\rangle}\right)_{0} \geq^{*} \cdot q^{\xi_{0}, \rho_{0}, \zeta} \frown p_{0}^{\prime \prime} \geq^{*} q^{\frown} r_{0} \in G .
$$

Hence

$$
q^{\xi_{0}, \rho_{0}, \zeta} \frown\left(\left(u^{\xi_{0}, \rho_{0}, \zeta}\right)_{\left\langle s \xi_{0}, \rho\right\rangle}\right)_{0} \in G
$$

Corollary 8.6. $P_{\bar{E}}$ is proper.

\section{Cardinals in $V^{P_{\bar{E}}}$}

Lemma 9.1. $\kappa^{+}$remains a cardinal in $V^{P_{\bar{E}}}$.

Proof. The proof really has no connection to the specific structure of $P_{\bar{E}}$. It is an exercise in properness.

Let

$$
\left.p \Vdash r \tilde{f}: \widehat{\kappa} \rightarrow \widehat{\kappa^{+}}\right\urcorner .
$$

Choose $\chi$ large enough so that $H_{\chi}$ contains everything we are interested in. Take $N \prec H_{\chi}$ such that

(1) $p, P_{\bar{E}}, \tilde{f} \in N$,

(2) $|N|=\kappa$,

(3) $N \supseteq V_{\kappa}$,

(4) $N \supseteq N^{<\kappa}$.

By Corollary 8.6 there is a $q \leq p$ that is $\left\langle N, P_{\bar{E}}\right\rangle$-generic. Let us set

$$
\lambda=N \cap \kappa^{+}
$$

hence $\lambda$ is an ordinal $<\kappa^{+}$.

Let $G$ be $P_{\bar{E}}$-generic with $q \in G$. The $\left\langle N, P_{\bar{E}}\right\rangle$-genericity ensures us that for all

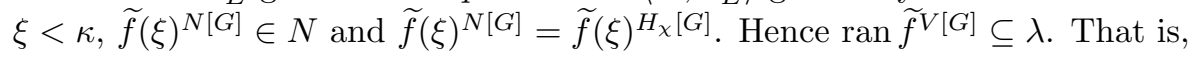

$$
q \Vdash\left\ulcorner\tilde{f} \text { is bounded in } \kappa^{+\urcorner}\right. \text {. }
$$

Lemma 9.2. No cardinals $>\kappa$ are collapsed by $P_{\bar{E}}$.

Proof. $\kappa^{+}$is not collapsed by Lemma 9.1. No cardinals $\geq \kappa^{++}$are collapsed, since $P_{\bar{E}}$ satisfies $\kappa^{++}$-c.c.

Lemma 9.3. Let $\xi<\kappa$, and let $\zeta$ be the ordinal such that $\kappa^{0}\left(\bar{E}_{G}(\zeta)\right) \leq \xi<$ $\kappa^{0}\left(\bar{E}_{G}(\zeta+1)\right)$. Then $\mathcal{P}(\xi) \cap V[G]=\mathcal{P}(\xi) \cap V[G\lceil\zeta]$.

Proof. Take $p=p_{n} \frown \ldots \frown p_{k+1} \frown p_{k} \frown \ldots \frown p_{0} \in G$ such that $\bar{E}\left(p_{k+1}\right)=\bar{E}_{G}(\zeta)$, $\bar{E}\left(p_{k}\right)=\bar{E}_{G}(\zeta+1)$. We know that $V[G]=V[G / p]$. So we work in $P_{\bar{E}} / p$. Set $p^{l}=$ $p_{n} \frown \ldots \frown p_{k+1}, p^{h}=\left\{\left\langle\bar{E}_{G}(\zeta), \emptyset\right\rangle\right\} \frown p_{k} \frown \ldots \frown p_{0}$. Then $P_{\bar{E}} / p=P_{\bar{E}}\left\lceil p^{l} \times P_{\bar{E}}\left\lceil p^{h}\right.\right.$. Note that $\left\langle P_{\bar{E}} / p^{h}, \leq^{*}\right\rangle$ is $\kappa^{0}\left(\bar{E}_{G}(\zeta+1)\right)$-closed. In particular, it is $\xi^{+}$-closed.

Let $A \in V[G], A \subseteq \xi$. Choose $\widetilde{A}$, a canonical $P_{\bar{E}} / p$-name for $A$. Let $q \in P_{\bar{E}} / p^{h}$. By induction we construct $\left\langle q_{\tau} \mid \tau<\xi\right\rangle$ satisfying 
(1) $\tau_{0}<\tau_{1} \Longrightarrow q_{\tau_{1}} \leq^{*} q_{\tau_{0}}$, and

(2) $q_{\tau} \|\ulcorner\widehat{\tau} \in \widetilde{A}\urcorner$.

Choose $q_{\xi} \leq^{*} q_{\tau}$ for all $\tau<\xi$.

By a density argument we can construct a $P\left\lceil p^{l}\right.$-name $\widetilde{B}$ such that $A=\widetilde{A}[G]=$ $\widetilde{B}\left[G\left\lceil p^{l}\right]\right.$.

Corollary 9.4. No cardinals $\leq \kappa$ are collapsed by $P_{\bar{E}}$.

Proof. Let $G \subseteq P_{\bar{E}}$ be generic. Assume $\lambda<\kappa$ is a collapsed cardinal. Let $\mu=$ $|\lambda|^{V[G]}$. We have $\mu<\lambda$, and there is an $A \in \mathcal{P}(\mu)^{V[G]}$ that codifies the order type $\lambda$. Let $\zeta$ be the unique ordinal such that $\kappa^{0}\left(\bar{E}_{G}(\zeta)\right) \leq \mu<\kappa^{0}\left(\bar{E}_{G}(\zeta+1)\right)$. By Lemma 9.3. $A \in V[G\lceil\zeta]$. Hence $\lambda$ is collapsed already in $V[G\lceil\zeta]$. However, by Lemma 9.2, $P_{\bar{E}_{G}(\zeta)}$ collapses no cardinals above $\kappa^{0}\left(\bar{E}_{G}(\zeta)\right)$, a contradiction.

So, no cardinal $<\kappa$ is collapsed. Since $\kappa$ is a limit of cardinals that are not collapsed, it is not collapsed.

We have just shown

Theorem 9.5. No cardinals are collapsed in $V^{P_{\bar{E}}}$.

\section{Properties of $\kappa$ IN $V^{P_{\bar{E}}}$}

Theorem 10.1. If $\mathrm{l}(\bar{E})=\kappa^{+}$, then $V^{P_{\bar{E}}} \vDash\ulcorner\kappa$ is regular .

Proof. Let $\lambda<\kappa$, and let $\tilde{f}$ be such that

$$
\Vdash_{P_{\bar{E}}}\ulcorner\widetilde{f}: \widehat{\lambda} \rightarrow \widehat{\kappa}\urcorner .
$$

Let

$$
D_{0}=\{p \mid \exists i p \Vdash\ulcorner\widetilde{f}(0)=\widehat{i}\urcorner\} .
$$

Since $D_{0}$ is a dense open set, we can invoke Theorem 6.1 to get $p^{\prime 0}, n_{0}, S^{0} \subset T^{p^{0}}$ such that

$$
\begin{aligned}
& \forall k<n_{0} \forall\left\langle\nu_{1}, \ldots, \nu_{k}\right\rangle \in S^{\prime 0} \exists \xi<\mathrm{l}(\bar{E}) \operatorname{Suc}_{S}\left(\left\langle\nu_{1}, \ldots, \nu_{k}\right\rangle\right) \in E_{\mathrm{mc}\left(p^{\prime 0}\right)}(\xi), \\
& \forall\left\langle\nu_{1}, \ldots, \nu_{n_{0}}\right\rangle \in S^{\prime 0}\left(p^{\prime 0}\right)_{\left\langle\nu_{1}, \ldots, \nu_{n_{0}}\right\rangle} \in D_{0} .
\end{aligned}
$$

Let us set

$$
A_{0}^{\prime}=\left\{\left(p^{\prime 0}\right)_{\left\langle\nu_{1}, \ldots, \nu_{n_{0}}\right\rangle} \mid\left\langle\nu_{1}, \ldots, \nu_{n_{0}}\right\rangle \in S^{\prime 0}\right\} .
$$

$A_{0}$ is an anti-chain. By shrinking $T^{p^{\prime 0}}$ as was done in the proof of Theorem 7.1 we can make $A_{0}$ into a maximal anti-chain below $p^{\prime 0}$. Since $\lambda<\kappa$ and $\left\langle P_{\bar{E}}^{*}, \leq^{*}\right\rangle$ is $\kappa$-closed, we can construct $\mathrm{a} \leq^{*}$-decreasing sequence

$$
p^{\prime 0} \geq^{*} p^{\prime 1} \geq^{*} \cdots \geq^{*} p^{\prime \tau} \geq^{*} \cdots, \quad \tau<\lambda,
$$

and $n_{\tau}, S^{\prime \tau} \subseteq T^{p^{\prime \tau}}$ such that

$$
\begin{aligned}
& \forall k<n_{\tau} \forall\left\langle\nu_{1}, \ldots, \nu_{k}\right\rangle \in S^{\prime \tau} \exists \xi<\mathrm{l}(\bar{E}) \operatorname{Suc}_{S^{\prime \tau}}\left(\left\langle\nu_{1}, \ldots, \nu_{k}\right\rangle\right) \in E_{\mathrm{mc}\left(p^{\prime \tau}\right)}(\xi), \\
& \forall\left\langle\nu_{1}, \ldots, \nu_{n_{\tau}}\right\rangle \in S^{\prime \tau} \exists i\left(p^{\prime \tau}\right)_{\left\langle\nu_{1}, \ldots, \nu_{n_{\tau}}\right\rangle} \Vdash\ulcorner\tilde{f}(\widehat{\tau})=\widehat{i}\urcorner
\end{aligned}
$$

and

$$
A_{\tau}^{\prime}=\left\{\left(p^{\prime \tau}\right)_{\left\langle\nu_{1}, \ldots, \nu_{n_{\tau}}\right\rangle} \mid\left\langle\nu_{1}, \ldots, \nu_{n_{\tau}}\right\rangle \in S^{\prime \tau}\right\}
$$

is a maximal anti-chain below $p^{\prime \tau}$. 
Let $p^{\prime} \leq^{*} p^{\prime \tau}$ for all $\tau<\lambda$. We set $S^{\tau}=\pi_{\mathrm{mc}\left(p^{\prime}\right), \operatorname{mc}\left(p^{\prime \tau)}\right.}^{-1}\left(S^{\prime \tau}\right)$, and take $p^{\tau}$ to be $p^{\prime}$ with $\pi_{\mathrm{mc}\left(p^{\prime}\right), \mathrm{mc}\left(p^{\prime \tau}\right)}^{-1}\left(T^{p^{\prime \tau}}\right)$ substituted for $T^{p^{\prime}}$ and maybe shrunken a bit so that

$$
A_{\tau}=\left\{\left(p^{\tau}\right)_{\left\langle\nu_{1}, \ldots, \nu_{n_{\tau}}\right\rangle} \mid\left\langle\nu_{1}, \ldots, \nu_{n_{\tau}}\right\rangle \in S^{\tau}\right\}
$$

is a maximal anti-chain below $p^{\tau}$.

Let $p \leq^{*} p^{\tau}$ for all $\tau<\lambda$, and let $\widetilde{g}$ be the following $P_{\bar{E}}$-name:

$$
\widetilde{g}=\bigcup_{\tau<\lambda}\left\{\left\langle\widehat{\langle\tau, i\rangle},\left(p^{\tau}\right)_{\left\langle\nu_{1}, \ldots, \nu_{n_{\tau}}\right\rangle}\right\rangle \mid A_{\tau} \ni\left(p^{\tau}\right)_{\left\langle\nu_{1}, \ldots, \nu_{n_{\tau}}\right\rangle} \Vdash\ulcorner\widetilde{f}(\tau)=\widehat{i}\urcorner\right\} .
$$

Then

$$
p \Vdash\ulcorner\widetilde{f}=\widetilde{g}\urcorner .
$$

Let $P^{*}$ be the following forcing notion:

$$
P^{*}=\left\{q \leq_{R} p \mid q \in P_{\bar{E}}\right\} .
$$

By Claim 5.2, $\left\langle P^{*}, \leq_{R}\right\rangle$ is sub-forcing of $\left\langle P_{\bar{E}} / p, \leq\right\rangle$. Hence, if $G$ is $P_{\bar{E}^{-}}$generic, then $G^{*}=G \cap P^{*}$ is $P^{*}$-generic. $\widetilde{g}$ is in fact a $P^{*}$-name, and, as can be seen from its definition, $\widetilde{g}[G]=\widetilde{g}\left[G^{*}\right] \in V\left[G^{*}\right]$. So in order to complete the proof it is enough to show that $\Vdash_{P^{*}}\ulcorner\widetilde{g}$ is bounded $\urcorner$.

By Claim 5.3 there is $r \in R_{\mathrm{mc}(p)}$ such that $P^{*} \simeq R_{\mathrm{mc}(p)} / r$. Now we use the following fact about Radin forcing: When the measure sequence is of length $\kappa^{+}, \kappa$ is regular in the generic extension. Necessarily, $\Vdash_{P^{*}}\ulcorner\widetilde{g}$ is bounded $\urcorner$.

Definition 10.2. We say that $\tau<\mathrm{l}(\bar{E})$ is a repeat point of $\bar{E}$ if $P_{\bar{E}}=P_{\bar{E} \mid \tau}$.

We note, again, our convention that when $\tau_{1}<\tau_{2}$ we have $P_{\bar{E} \mid \tau_{2}} \subseteq P_{\bar{E} \mid \tau_{1}}$. The equality in the above definition is meant to be with this convention. We point out that if $\tau$ is a repeat point, then $P_{\bar{E} \mid \tau} \in M$.

Theorem 10.3. If $\bar{E}$ has a repeat point, then $V^{P_{\bar{E}}} \vDash\ulcorner\kappa$ is measurable .

Proof. Let $\tau$ be a repeat point of $\bar{E}$. The crucial observation regarding $\tau$ being a repeat point is as follows. Let $p=p_{n . .0} \in P_{\bar{E}}$. Set a function $f(\bar{\nu})=p_{\langle\bar{\nu}\rangle n+1 \ldots 1}$ for all $\langle\bar{\nu}\rangle \in T^{p_{0}}$. Then $j(f)(\operatorname{mc}(p) \mid \tau) \in P_{\bar{E} \mid \tau}$. By our convention this means $j(f)\left(\operatorname{mc}(p)\lceil\tau) \in P_{\bar{E}}\right.$. Moreover, $j(f)(\operatorname{mc}(p)\lceil\tau)=p$ !

We start by showing that for each $p \in P_{\bar{E}}, \Vdash_{P_{\bar{E}}}\ulcorner\widetilde{A} \subseteq \widehat{\kappa}\urcorner$ there is $p^{*} \leq^{*} p$ such that $j\left(p^{*}\right)_{\left\langle\operatorname{mc}\left(p^{*}\right) \mid \tau\right\rangle} \|_{j\left(P_{\bar{E}}\right)}\left\ulcorner\widehat{\kappa} \in j(\widetilde{A})^{\urcorner}\right.$. Note that $j(p)_{\langle\operatorname{mc}(p) \mid \tau\rangle}=p \frown\left(j(p)_{\langle\operatorname{mc}(p) \mid \tau\rangle}\right)_{0}$.

Choose $\chi$ large enough so that $H_{\chi}$ contains everything we are interested in. Take $N \prec H_{\chi}$ such that $p, \widetilde{A}, P_{\bar{E}} \in N,|N|=\kappa, N \supseteq V_{\kappa}, N \supseteq N^{<\kappa}$. By Corollary 8.6 there is a $p^{\prime} \leq^{*} p$ that is $\left\langle N, P_{\bar{E}}\right\rangle$-generic.

Let us fix $\langle\bar{\nu}\rangle \in T^{p^{\prime}}$. By Theorem[7.1 there is a $q \leq^{*} p_{\langle\bar{\nu}\rangle}^{\prime}$ such that $q \|_{P_{\bar{E}}}\left\ulcorner\kappa^{0}(\bar{\nu}) \in\right.$ $\widetilde{A}^{\urcorner}$. Then, by the $N$-genericity of $p^{\prime}$, there is $q^{\prime} \in N, q^{\prime} \leq^{*} p_{\left\langle\pi_{\operatorname{mc}\left(p^{\prime}\right) \operatorname{mc}(p)}(\nu)\right\rangle}, q^{\prime} \| q$, $q^{\prime} \|_{P_{\bar{E}}}\left\ulcorner\kappa^{0}(\bar{\nu}) \in \widetilde{A}^{\urcorner}\right.$. Hence there is a $q^{*} \leq^{*} q^{\prime}, p_{\langle\bar{\nu}\rangle}^{\prime}$ such that

$$
\operatorname{supp} q_{0}^{*}=\operatorname{supp} p_{0}^{\prime}, \quad q^{*} \|_{P_{\bar{E}}}\left\ulcorner\kappa^{0}(\bar{\nu}) \in \widetilde{A}\right\urcorner .
$$

So, for each $\langle\bar{\nu}\rangle \in T^{p^{\prime}}$ let $q^{*}(\bar{\nu}) \leq^{*} p_{\langle\bar{\nu}\rangle}^{\prime}$ be such that

$$
\operatorname{supp} q_{0}^{*}(\bar{\nu})=\operatorname{supp} p_{0}^{\prime}, \quad q^{*}(\bar{\nu}) \|_{P_{\bar{E}}}\left\ulcorner\kappa^{0}(\bar{\nu}) \in \widetilde{A}^{\urcorner} .\right.
$$

Of course this means $j\left(q^{*}\right)\left(\operatorname{mc}\left(p^{\prime}\right)\lceil\tau) \|_{j\left(P_{\bar{E}}\right)}\ulcorner\kappa \in j(\widetilde{A})\urcorner\right.$. Let us set $r_{n . .0}=$ $j\left(q^{*}\right)\left(\operatorname{mc}\left(p^{\prime}\right) \mid \tau\right) \in j\left(P_{\bar{E}}\right)$. By our convention $r_{n . .1} \in P_{\bar{E}}$. Let us set $p^{*}=r_{n . .1}$. We 
have $j\left(p^{*}\right)_{\left\langle\mathrm{mc}\left(p^{*}\right) \mid \tau\right\rangle} \leq^{*} j\left(q^{*}\right)\left(\operatorname{mc}\left(p^{\prime} \mid \tau\right)\right)$; hence $j\left(p^{*}\right)_{\left\langle\operatorname{mc}\left(p^{*}\right) \mid \tau\right\rangle} \|_{j\left(P_{\bar{E}}\right)}\ulcorner\kappa \in j(\widetilde{A})\urcorner$, as needed.

Let $\widetilde{U}$ be a $P_{\bar{E}}$-name defined by

$p \Vdash_{P_{\bar{E}}}\left\ulcorner\widetilde{A} \in \widetilde{U}^{\urcorner}\right.$if $\left\{q \in P_{\bar{E}} \mid j(q)_{\langle\operatorname{mc}(q) \mid \tau\rangle} \Vdash_{j\left(P_{\bar{E}}\right)}\left\ulcorner\kappa \in j(\widetilde{A})^{\urcorner}\right\}\right.$is dense below $p$.

We note that, since $\left\{q \in P_{\bar{E}} \mid j(q)_{\langle\operatorname{mc}(q) \mid \tau\rangle} \|_{j\left(P_{\bar{E}}\right)}\left\ulcorner\kappa \in j(\widetilde{A})^{\urcorner}\right\}\right.$is dense, we have $p \Vdash_{P_{\bar{E}}}\ulcorner\widetilde{A} \notin \widetilde{U}\urcorner$ if $\left\{q \in P_{\bar{E}} \mid j(q)_{\langle\operatorname{mc}(q) \mid \tau\rangle} \Vdash_{j\left(P_{\bar{E}}\right)}\left\ulcorner\kappa \notin j(\widetilde{A})^{\urcorner}\right\}\right.$is dense below $p$.

We show that $\widetilde{U}$ is a name for a measure on $\kappa$ :

(1) $\Vdash_{P_{\bar{E}}}\ulcorner\emptyset \notin \widetilde{U}\urcorner$ : Immediate due to $\Vdash_{j\left(P_{\bar{E}}\right)}\ulcorner\kappa \notin j(\emptyset)\urcorner$.

(2) $p \Vdash_{P_{\bar{E}}}\ulcorner\widetilde{A} \notin \widetilde{U}\urcorner \Longrightarrow p \Vdash_{P_{\bar{E}}}\left\ulcorner\kappa \backslash \widetilde{A} \in \widetilde{U}^{\urcorner}\right.$: So, there is a maximal antichain $X$ below $p$ such that $q \in X \Longrightarrow j(q)_{\langle\mathrm{mc}(q) \mid \tau\rangle} \Vdash_{j\left(P_{\bar{E}}\right)}\ulcorner\kappa \notin j(\widetilde{A})\urcorner$. Then $q \in X$ implies $j(q)_{\langle\operatorname{mc}(q) \mid \tau\rangle} \Vdash_{j\left(P_{\bar{E}}\right)}\ulcorner\kappa \in j(\kappa \backslash \widetilde{A})\urcorner$. This means $p \Vdash_{P_{\bar{E}}}\ulcorner\kappa \backslash \widetilde{A} \in \widetilde{U}\urcorner$.

(3) $p \Vdash\ulcorner\widetilde{A} \in \widetilde{U}, \widetilde{A} \subseteq \widetilde{B}\urcorner \Longrightarrow p \Vdash\ulcorner\widetilde{B} \in \widetilde{U}\urcorner$ : Obvious.

(4) $\lambda<\kappa, p \Vdash_{P_{\bar{E}}}\left\ulcorner\forall \xi<\lambda \widetilde{A}_{\xi} \in \widetilde{U}^{\urcorner} \Longrightarrow p \Vdash_{P_{\bar{E}}}\left\ulcorner\cap_{\xi<\lambda} \widetilde{A}_{\xi} \in \widetilde{U}^{\urcorner}\right.\right.$: So, for all $\xi<\lambda$ there is a maximal anti-chain $X_{\xi}$ below $p$ such that $q \in X_{\xi} \Longrightarrow$ $j(q)_{\langle\operatorname{mc}(q) \mid \tau\rangle} \Vdash_{j\left(P_{\bar{E}}\right)}\left\ulcorner\kappa \in j\left(\widetilde{A}_{\xi}\right)\right\urcorner$. It is enough to show that $\left\{r \in P_{\bar{E}} \mid\right.$ $\left.j(r)_{\langle\mathrm{mc}(r) \mid \tau\rangle} \Vdash_{j\left(P_{\bar{E}}\right)}\left\ulcorner\forall \xi<\lambda \kappa \in j\left(\widetilde{A}_{\xi}\right)\right\urcorner\right\}$ is dense below $p$.

Let $q=q_{n . .0} \leq p$. Since $\left\{r \in P_{\bar{E}} \mid j(r)_{\langle\operatorname{mc}(r) \mid \tau\rangle} \Vdash_{j\left(P_{\bar{E}}\right)}\left\ulcorner\kappa \in j\left(\widetilde{A}_{\xi}\right)^{\urcorner}\right\}\right.$is dense below $p$ for all $\xi<\lambda$, we can construct $\left\langle r^{\xi} \mid \xi \leq \lambda\right\rangle,\left\langle Y_{\xi} \mid \xi<\lambda\right\rangle$ such that:

- $\xi=0: r^{0}=q_{0}$,

- $\xi$ is a limit ordinal: $\forall \xi^{\prime}<\xi r^{\xi} \leq^{*} r^{\xi^{\prime}}$,

- $\xi=\xi^{\prime}+1: r^{\xi} \leq^{*} r^{\xi^{\prime}}, Y_{\xi}$ is a maximal anti-chain below $q_{n . .1}$, and, for all $s \in Y_{\xi}, s \frown j\left(r^{\xi}\right)_{\langle\operatorname{mc}(q) \mid \tau\rangle} \Vdash_{j\left(P_{\bar{E}}\right)}\left\ulcorner\kappa \in j\left(\widetilde{A}_{\xi^{\prime}}\right)\right\urcorner$.

Then we have $q_{n . .1} \frown j\left(r^{\lambda}\right)_{\left\langle\mathrm{mc}\left(r^{\lambda}\right) \mid \tau\right\rangle} \Vdash_{j\left(P_{\bar{E}}\right)}\left\ulcorner\forall \xi<\lambda \kappa \in j\left(\widetilde{A}_{\xi}\right)^{\urcorner}\right.$.

We note that actually the measure $\widetilde{U}$ constructed in the above proof is normal. Moreover, we can construct an extender, lifting $E(0)$, by deciding formulas of the form $\alpha \in j(\widetilde{A})$ for $\alpha \in \operatorname{dom} E(0)$.

\section{What Have We Proved?}

We can sum everything up as follows:

We can control independently two properties of $\kappa$ in a generic extension. The first is the size of $2^{\kappa}$, which is controlled by $|\bar{E}|$. The second is how "big" we want $\kappa$ to be, which is controlled by $\mathrm{l}(\bar{E})$.

\section{Generic by Iteration}

Recall that if $R$ is Radin forcing generated from $j: V \rightarrow M$, then there are $\tau$ and $G \in V$ such that $G$ is $j_{0, \tau}(R)$-generic over $M_{\tau}$.

Our original aim was to find some form of this claim for our forcing. We have a partial result in this direction. Namely, when $l(\bar{E})=1$ we have a generic filter in $V$ over an elementary submodel in $M_{\omega}$.

In this section we assume that $l(\bar{E})=1$. 
Let us take an iteration of $j=j_{0,1}$,

$$
\left\langle\left\langle M_{n} \mid n<\omega\right\rangle,\left\langle j_{n, m} \mid n \leq m<\omega\right\rangle\right\rangle .
$$

Choose $\chi$ large enough so that everything interesting is in $H_{\chi}$ (i.e., $P_{\bar{E}} \in H_{\chi}$ ), and set

$$
\begin{aligned}
& \kappa_{n}=j_{0, n}(\kappa), \\
& \bar{E}^{n}=j_{0, n}(\bar{E}), \\
& P^{n}=j_{0, n}\left(P_{\bar{E}}\right)\left(=P_{\bar{E}^{n}}\right), \\
& \chi_{n}=j_{0, n}(\chi) .
\end{aligned}
$$

Definition 12.1 (when $\mathrm{l}(\bar{E})=1$ ). We call $\langle N, p\rangle$ a $k$-pair if

(1) $M_{k} \vDash\left\ulcorner N \prec H_{\chi_{k}}^{\left.M_{k}\right\urcorner}\right.$,

(2) $M_{k} \vDash\left\ulcorner|N|=\kappa_{k}\right.$,

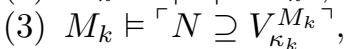

(4) $M_{k} \vDash\left\ulcorner N \supseteq N^{\left.<\kappa_{k}\right\urcorner}\right.$

(5) $p \in P^{k+1} \cap j_{k, k+1}(N)$, and

(6) if $D \in N$ is dense open in $P^{k}$, then there are $n, S \leq T^{p}$ such that

$$
\forall\left\langle\nu_{1}, \ldots, \nu_{n}\right\rangle \in S(p)_{\left\langle\nu_{1}, \ldots, \nu_{n}\right\rangle} \in j_{k, k+1}(D) .
$$

Claim 12.2. Let $N \in M_{k}, k<\omega$ and $q \in j_{k, k+1}(N) \cap P^{k+1}$ be such that $M_{k}$ satisfies

(1) $N \prec H_{\chi_{k}}^{M_{k}}$,

(2) $|N|=\kappa_{k}$,

(3) $N \supseteq V_{\kappa_{k}}^{M_{k}}$,

(4) $N \supseteq N^{<\kappa_{k}}$

(5) $P^{k} \in N$.

Then there is a $p \in j_{k, k+1}(N) \cap P^{k+1}$ such that

(1) $p \leq^{*} q$, and

(2) $\langle N, p\rangle$ is a k-pair.

Proof. Let $\left\langle D_{\xi}^{k} \mid \xi<\kappa_{k}\right\rangle$ be an enumeration, in $M_{k}$, of all the dense open subsets of $P^{k}$ that are in $N$. Set $N_{k+1}=j_{k, k+1}(N)$. Since we have

$$
\begin{aligned}
& \left\langle j_{k, k+1}\left(D_{\xi}^{k}\right) \mid \xi<\kappa_{k}\right\rangle \subseteq N_{k+1}, \\
& \left\langle j_{k, k+1}\left(D_{\xi}^{k}\right) \mid \xi<\kappa_{k}\right\rangle \in M_{k+1}, \\
& M_{k+1} \vDash\left\ulcorner N_{k+1} \supseteq N_{k+1}^{\left.<\kappa_{k+1}\right\urcorner},\right.
\end{aligned}
$$

we get that

$$
\left\langle j_{k, k+1}\left(D_{\xi}^{k}\right) \mid \xi<\kappa_{k}\right\rangle \in N_{k+1} .
$$

Starting with $q$, we construct in $N_{k+1}$ a $\leq^{*}$-decreasing sequence $\left\langle p_{\xi} \mid \xi<\kappa_{k}\right\rangle$ using Theorem 6.1 Note that we have no problem at the limit stages, since $N_{k+1} \vDash$ $\left\ulcorner\left\langle P^{k+1}, \leq^{*}\right\rangle\right.$ is $\kappa_{k+1}$-closed $\urcorner$. Now choose $p \in P^{k+1} \cap N_{k+1}$ such that $\forall \xi<\kappa_{k} p \leq^{*}$ $p_{\xi}$. We get that $\langle N, p\rangle$ is a $k$-pair.

Definition 12.3. We call $\langle\vec{N}, \vec{p}\rangle$ a $P^{\omega}$-generic approximation sequence if

$$
\langle\vec{N}, \vec{p}\rangle=\left\langle\left\langle N_{k}, p^{k+1}\right\rangle \mid k_{0} \leq k<\omega\right\rangle
$$

and for all $k_{0} \leq k<\omega$, 
(1) $M_{k} \vDash\left\ulcorner N_{k} \prec H_{\chi_{k}}^{\left.M_{k}\right\urcorner}\right.$,

(2) $M_{k} \vDash\left\ulcorner\left|N_{k}\right|=\kappa_{k}\right\urcorner$,

(3) $M_{k} \vDash\left\ulcorner N_{k} \supseteq V_{\kappa_{k}}^{\left.M_{k}\right\urcorner}\right.$

(4) $M_{k} \vDash\left\ulcorner N_{k} \supseteq N^{<\kappa_{k}}\right\urcorner$,

(5) $P^{k} \in N_{k}$

(6) $\left\langle N_{k}, p^{k+1}\right\rangle$ is a $k$-pair,

(7) $j_{k_{1}, k_{2}}\left(N_{k_{1}}\right)=N_{k_{2}}$,

(8) $p^{k+2} \leq^{*}\left(j_{k+1, k+2}\left(p^{k+1}\right)\right)_{\left\langle\operatorname{mc}\left(p^{k+1}\right)\right\rangle}$.

Definition 12.4. Let $\langle\vec{N}, \vec{r}\rangle$ be a $P^{\omega}$-generic approximating sequence. Then

$$
G(\langle\vec{N}, \vec{r}\rangle)=\left\{p \in P^{\omega} \mid \exists k j_{k, \omega}\left(r^{k}\right) \leq^{*} p\right\} .
$$

Claim 12.5. Let $k_{0}<\omega, q \in P^{k_{0}} \cap N_{k_{0}}$, and assume for all $k_{0} \leq k<\omega$,

(1) $P^{k} \in N_{k}$,

(2) $j_{k, k+1}\left(N_{k}\right)=N_{k+1}$,

(3) $M_{k} \vDash\left\ulcorner N_{k} \prec H_{\left.\chi_{k}\right\urcorner}^{\left.M_{k}\right\urcorner}\right.$,

(4) $M_{k} \vDash\left\ulcorner\left|N_{k}\right|=\kappa_{k}\right\urcorner$,

(5) $M_{k} \vDash\left\ulcorner N_{k} \supseteq V_{\kappa_{k}}^{\left.M_{k}\right\urcorner}\right.$

(6) $M_{k} \vDash\left\ulcorner N_{k} \supseteq N_{k}^{<<}\right\urcorner$.

Then there is a $P^{\omega}$-generic approximating sequence

$$
\langle\vec{N}, \vec{p}\rangle=\left\langle\left\langle N_{k}, p^{k+1}\right\rangle \mid k_{0} \leq k<\omega\right\rangle
$$

such that $p^{k_{0}+1} \leq^{*} j_{k_{0}, k_{0}+1}(q)$.

Proof. We construct the $p^{k+1}$ by induction. We set $p^{k_{0}}=q$.

Assume that we have constructed $\left\langle p^{k^{\prime}} \mid k_{0} \leq k^{\prime} \leq k\right\rangle$. We set $q^{k+1}=$ $j_{k, k+1}\left(p^{k}\right)_{\left\langle\mathrm{mc}\left(p^{k}\right)\right\rangle}$. Invoke Claim 12.2 to get $p^{k+1} \leq^{*} q^{k+1}$ such that $\left\langle N_{k}, p^{k+1}\right\rangle$ is a $k$-pair.

When the induction terminates we have $\left\langle\left\langle N_{k}, p^{k+1}\right\rangle \mid k_{0} \leq k<\omega\right\rangle$, as required.

Claim 12.6. Let $k_{0}<\omega$, and assume that

(1) $M_{k_{0}} \vDash\left\ulcorner N_{k_{0}} \prec H_{\chi_{k_{0}}}^{\left.M_{k_{0}}\right\urcorner}\right.$

(2) $M_{k_{0}} \vDash\left\ulcorner\left|N_{k_{0}}\right|=\kappa_{k_{0}}\right\urcorner$,

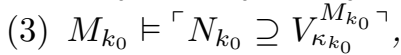

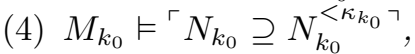

(5) $P^{k_{0}} \in N_{k_{0}}$,

(6) $q \in P^{k_{0}} \cap N_{k_{0}}$.

Then there is a $P^{\omega}$-generic approximating sequence

$$
\langle\vec{N}, \vec{p}\rangle=\left\langle\left\langle N_{k}, p^{k+1}\right\rangle \mid k_{0} \leq k<\omega\right\rangle
$$

such that $p^{k_{0}+1} \leq^{*} j_{k_{0}, k_{0}+1}(q)$.

Proof. We set $N_{k}=j_{k_{0}, k}\left(N_{k_{0}}\right)$ for all $k_{0}<k<\omega$, and then we invoke Claim 12.5.

Theorem 12.7. Assume

(1) $M_{\omega} \vDash\left\ulcorner N \prec H_{\chi_{\omega}}^{\left.M_{\omega}\right\urcorner}\right.$,

(2) $M_{\omega} \vDash\left\ulcorner|N|=\kappa_{\omega}\right\urcorner$, 


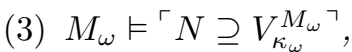

(4) $M_{\omega} \vDash\left\ulcorner N \supseteq N^{\left.<\kappa_{\omega}\right\urcorner}\right.$,

(5) $P^{\omega} \in N$,

(6) $q \in P^{\omega} \cap N$.

Then there is, in $V$, a filter $G \subseteq P^{\omega}$ such that

(1) $q \in G$, and

(2) $\forall D \in N D$ is dense open in $P^{\omega} \Longrightarrow G \cap D \cap N \neq \emptyset$.

Proof. We find $\langle\vec{N}, \vec{p}\rangle$, a $P^{\omega}$-generic approximating sequence. $G(\langle\vec{N}, \vec{p}\rangle)$ is the required filter.

Find $k_{0}$ and $N_{k_{0}}, q^{k_{0}}$ such that

$$
\begin{aligned}
& P^{k_{0}} \in N_{k_{0}}, \\
& j_{k_{0}, \omega}\left(N_{k_{0}}\right)=N, \\
& j_{k_{0}, \omega}\left(q^{k_{0}}\right)=q .
\end{aligned}
$$

Invoke Claim 12.6 to get from $N^{k_{0}}$ and $q^{k_{0}}$ a generic approximating sequence $\langle\vec{N}, \vec{p}\rangle$.

Let $D \in N$ be dense open in $P^{\omega}$.

Find $k \geq k_{0}$ and $D^{k}$ such that $j_{k, \omega}\left(D^{k}\right)=D$. As usual, set $D^{k+l}=j_{k, k+l}\left(D^{k}\right)$. By construction there is an $n$ such that

$$
\forall\left\langle\nu_{1}, \ldots, \nu_{n}\right\rangle \in T^{p^{k+1}}\left(p^{k+1}\right)_{\left\langle\nu_{1}, \ldots, \nu_{n}\right\rangle} \in D^{k+1} \cap N_{k+1},
$$

which means that

$$
j_{k+1, k+1+n}\left(p^{k+1}\right)_{\left\langle\mathrm{mc}\left(p^{k+1}\right), \ldots, j_{k+1, k+n}\left(\operatorname{mc}\left(p^{k+1}\right)\right)\right\rangle} \in D^{k+1+n} \cap N_{k+1+n} .
$$

Hence

$$
j_{k+1, \omega}\left(p^{k+1}\right)\left\langle\operatorname{mc}\left(p^{k+1}\right), \ldots, j_{k+1, k+n}\left(\operatorname{mc}\left(p^{k+1}\right)\right)\right\rangle \in D \cap N .
$$

Since $\langle\vec{N}, \vec{p}\rangle$ is a $P^{\omega}$-generic approximation sequence, it satisfies

$$
p^{k+1+n} \leq^{*} j_{k+1, k+1+n}\left(p^{k+1}\right)_{\left\langle\operatorname{mc}\left(p^{k+1}\right), \ldots, j_{k+1, k+1+n}\left(\operatorname{mc}\left(p^{k+1}\right)\right)\right\rangle} .
$$

Hence

$$
j_{k+1+n, \omega}\left(p^{k+1+n}\right) \leq^{*} j_{k+1, \omega}\left(p^{k+1}\right)_{\left\langle\operatorname{mc}\left(p^{k+1}\right), \ldots, j_{k+1, k+n}\left(\operatorname{mc}\left(p^{k+1}\right)\right)\right\rangle},
$$

giving us that

$$
j_{k+1, \omega}\left(p^{k+1}\right)_{\left\langle\operatorname{mc}\left(p^{k+1}\right), \ldots, j_{k+1, k+n}\left(\operatorname{mc}\left(p^{k+1}\right)\right)\right\rangle} \in G(\langle\vec{N}, \vec{p}\rangle) ;
$$

so $G(\langle\vec{N}, \vec{p}\rangle) \cap D \cap N \neq \emptyset$ by (12.7.1) and (12.7.2).

\section{Concluding Remarks}

(1) A definition of repeat point that depends only on the extender sequence and is equivalent to the one we gave (which mentions $P_{\bar{E}}$ ) will probably be useful.

(2) It is not completely clear what $1(\bar{E})$ should be in order to make sure that $\bar{E}$ has a repeat point.

(3) A finer analysis in the case of measurability and stronger properties is needed - for example, extending the elementary embedding to the generic extension, and not just constructing a normal ultrafilter.

(4) We do not know how to get a generic by iteration when $l(\bar{E})>1$. 
(5) Making this forcing more "precise" by adding "gentle" collapses so that we get a prescribed behaviour on all cardinals below $\kappa$ in the generic extension is in preparation.

\section{REFERENCES}

[1] J. Cummings, A Model in which GCH Holds at Successors but Fails at Limits, Transactions of the American Mathematical Society 329(1992), Number 1, 1-39 MR 92h:03076

[2] M. Magidor, Changing Cofinality Of Cardinals, Fundamenta Mathematicae 99 (1978), 61-71 MR 57:5754

[3] M. Gitik and M. Magidor, The Singular Cardinal Hypothesis Revisited, in Set Theory of the Continuum, H. Judah, W. Just, H. Woodin, (Eds.), MSRI Publ., vol. 26, Springer-Verlarg (1992), 243-278 MR 95c:03131

[4] M. Gitik, Changing Cofinalities and the Non-Stationary Ideal, Israel Journal of Mathematics 56 (1986), 280-314 MR 89b:03086

[5] W. Mitchell, How Weak is a Closed Unbounded Ultrafilter?, in Logic Colloquium '80, D. van Dalen, D. Lascar, J. Smiley, (Eds.), North-Holland Publishing Company (1982), 209-230 MR 84f:03047

[6] K. Prikry, Changing measurable into accessible cardinals, Diss. Math. 68 (1970), 5-52 MR 41:6685

[7] L. B. Radin, Adding Closed Cofinal Sequences to Large Cardinals, Annals of Mathematical Logic 22(1982), 243-261 MR 83m:03062

[8] M. Segal, On Powers of Singular Cardinals with Cofinality $>\omega$, Master's Thesis, The Hebrew University of Jerusalem (1995)

[9] S. Shelah, Proper Forcing, Lecture Notes in Mathematics, Vol. 940, Springer, Berlin, 1982 MR 84h:03002

[10] H. Woodin and J. Cummings, Chapters from an unpublished book on Radin forcing

Computer Science Department, The Academic College of Tel-Aviv, 4 Antokolsky St., Tel-Aviv 64044, IsRael

E-mail address: carmi@mta.ac.il 